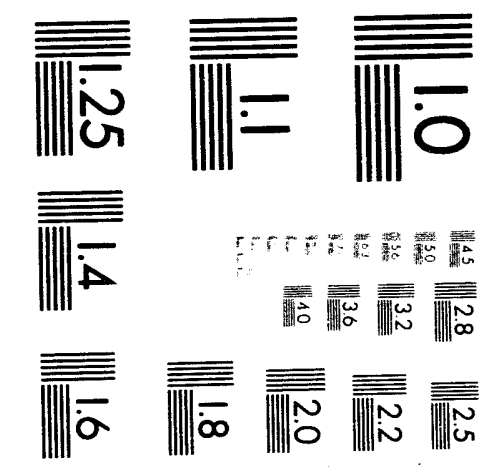



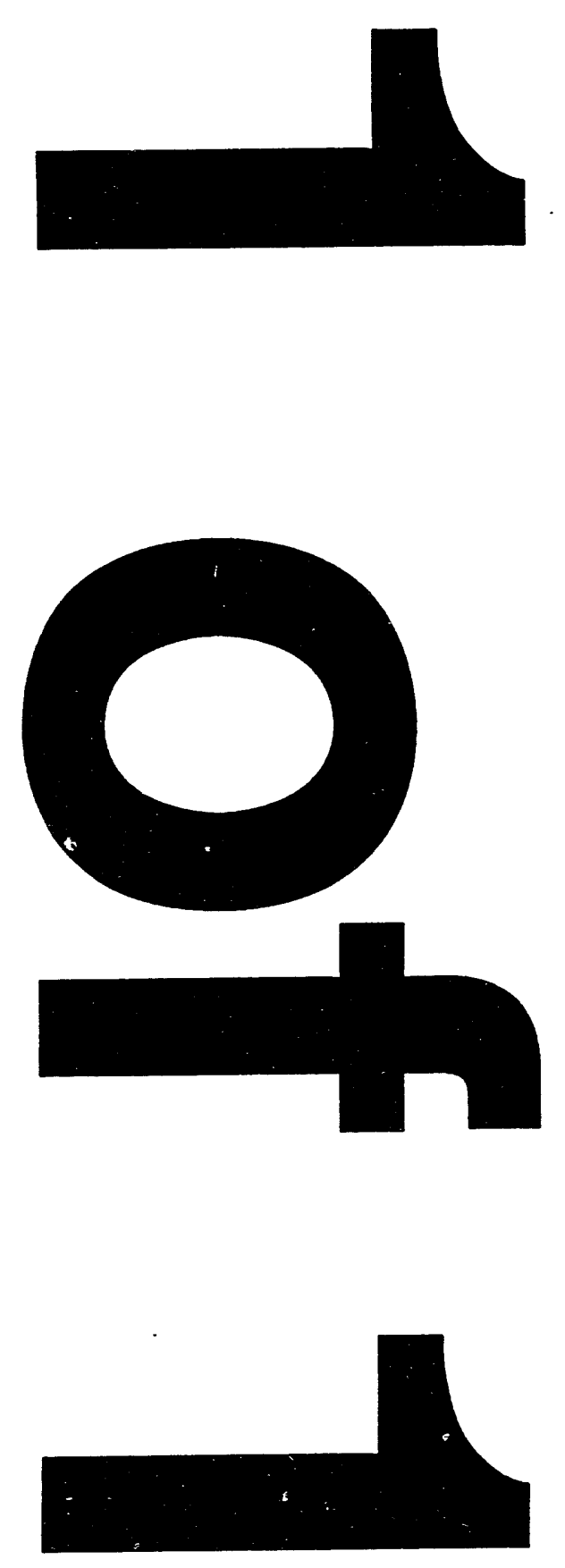


\title{
K-effective CALCULATIONS FOR INFINITE PLANAR SQUARE-PITCHED ARRAYS OF WASTE RECEPTACLES
}

\author{
B. A. Pohl
}

B. L. Koponen

September 27, 1993

This is an informal report intended primarily for internal or limited external distribution. The opinions and conclusions stated are thosn of the author and may or inay not be those of the Laboratory.

Work performed under the auspices of the U.S. Department of Energy by the Lawrence Livermore National Laboratory under Contract W-7405-Eng-48.

.

(1) 


\section{DISCLAIMER}

This document was prepared as an account of work sponsored by an agency of the United States Government. Neither the United States Government nor the University of Califomia nor any of their employees, makes any warranty, express or implied, or assumes any legal liability or responsibility for the accuracy, completeness, or usefulness of any information, apparatus, product, or process disclosed, or represents that its use would not infringe privately owned rights. Reference herein to any specific commercial products, process, or service by trade name, trademark, manufacturer, or otherwise, does not necessarily constitute or imply its endorsement, recommendation, or favoring by the United States Government or the University of California. The views and opinions of authors expressed herein do not necessarily state or reflect those of the United States Government or the University of California, and shall not be used for advertising or product endorsement purposes.

This report has been reproduced directly from the best available copy.

Available to DOE and DOE contractors from the Oflice of Scientific and Technical Information

P.O. Box 62, Oak Ridge, TN 37831

Prices available from (615) 576-8401, FTS 626-8401

A vailable to the public from the

National Technical Information Service

U.S. Department of Commerce

5285 Port Royal Rd.,

Springfield, VA 22161 


\section{TABLE OF CONTENTS}

Textual Body of Report

Figures

Graphical Representation of the Results

Appendix 1 Materials Specification

Appendix 2 Typical SCALE Input Files

Appendix 3 Tabular Representation of the Results

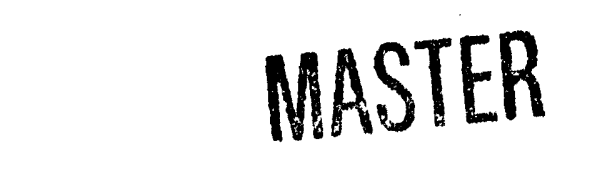




\title{
Keffective CALCULATIONS FOR INFINITE PLANAR SQUARE-PITCHED ARRAYS OF WASTE RECEPTACLES
}

\author{
B.A. Pohl and B.L. Koponen \\ Lawrence Livermore National Laboratory
}

\begin{abstract}
Neutron multiplication factors $\left(\mathrm{k}_{\mathrm{eff}}\right)$ have been calculated for arrays of three standard sizes of waste receptacles: 5,30 , and 55 gallon drums containing either $2 \%$ or $5 \%$ enriched uranium compounds mixed with either water or oil. The calculations demonstrate the effect of both the array pitch and the uranium concentration on $\mathbf{k}_{\mathrm{eff}}$ No container was subcritical for all possible sets of concentration and pitch. All were safe for both very low and very high uranium concentrations. Accident condition calculations, for which an extra drum is added to the system, show little effect on criticality.
\end{abstract}

\section{CONTAINER ARRAY GEOMETRY}

Carbon steel drums that would contain either a $\mathrm{UO}_{2} \mathrm{~F}_{2}$-water solution or a UF 4 -oil mixture are modeled as right cylinders. The containers and the air surrounding them are contained in a region directly above a horizontal slab of concrete flooring in a room with a ceiling eight feet high. The ceiling is modeled as a thick layer of water; the air in the room is modeled as a low density water mist. A unit cuboid is constructed from a three-element array containing the floor slab; the region between the floor and the ceiling containing the drums; and the ceiling slab.(Fig. 1) This unit cell is specularly reflected on the four of its vertical faces to effect an infinite planar square horizontal array. The array spacing of the containers (pitch) is determined by the horizontal dimensions of this unit cell. This system is treated as a squarepitch latticecell in which the drum walls are the cladding and the moist air, the moderator.

\section{COMPUTER SYSTEMS}

Throughout this study we used KENO V.a - CSAS4 in SCALE4.1, with the 27-group cross section library. Part of the calculations were remotely executed on the configuration controlled NCSS IBM 3090 system maintained by the Computing and Telecommunications Division of Martin Marietta Energy Systems, Inc. at Oak Ridge, Tennessee [Ref. 1] via INTERNET links to LLNL. Due to budget considerations, many of the calculations were run locally using the IBM PC 386/486 version of CSAS4 developed at Idaho National Engineering Laboratory [Ref. 2]. Figure 2 indicates which computer system was used for 
each of the data sets and references the labeling scheme we used to index the calculations. Sufficient histories were run to obtain a typical error of less than $0.3 \%$; this represented $\sim 10$ minutes per data point on the IBM 3090 and $\sim 1$ hour per point of PC time. Over 1000 data points were computed during this study.

\section{DRUM DIMENSIONS}

We modeled each drum as a nested set of two right circular cylinders; the skin thickness of the containers being derived from the differences in the heights and diameters of the two cylinders. The dimensions for the 5, 30, and 55 gallon drums to be incorporated in these models follow from ANSI standards [Ref. 3]. In instances where a choice of parameters needed to be made from a range of values, we adopted suggested values [Ref. 4]. These choices involved selection of the thickness of the carbon steel stock from a set of possible DOT Designated Gages, and the definition of the effective height of the drum. The ambiguity in defining the heights is due to typical drum construction where of the upper and lower lid of the drums are not true planes and do not necessarily intersect the cylindrical shell at its full length (rim height). The amount of the uncertainty is different for each size of drum and was dealt with individually. It should be noted that at the aspect ratio of all of the drums, small changes to the height of the drums has little effect on reactivity; for elongated cylinders the diameter is the sensitive parameter. There was no uncertainty in the diameters of the drums. The applicable parameters are listed below:

\begin{tabular}{|c||c|c||c|c||c|c|}
\hline $\begin{array}{c}\text { nominal } \\
\text { container } \\
\text { size }\end{array}$ & $\begin{array}{c}\text { range of } \\
\text { steel } \\
\text { gages }\end{array}$ & $\begin{array}{c}\text { selected } \\
\text { steel } \\
\text { gage }\end{array}$ & $\begin{array}{c}\text { range of } \\
\text { inside } \\
\text { diameters }\end{array}$ & $\begin{array}{c}\text { selected } \\
\text { inside } \\
\text { diameter }\end{array}$ & $\begin{array}{c}\text { range of } \\
\text { inside } \\
\text { heights }\end{array}$ & $\begin{array}{c}\text { selected } \\
\text { inside } \\
\text { height }\end{array}$ \\
\hline 5 Gallon & $\begin{array}{c}24 \\
\text { to } \\
28\end{array}$ & 28 & $11.25^{\prime \prime}$ & $11.25^{\prime \prime}$ & $\begin{array}{c}12.8125^{\prime \prime} \\
\text { to } \\
13.6250^{\prime \prime}\end{array}$ & $13.4375^{\prime \prime}$ \\
\hline 30 Gallon & $\begin{array}{c}12 \\
\text { to } \\
18\end{array}$ & 18 & $18.25^{\prime \prime}$ & $18.25^{\prime \prime}$ & $\begin{array}{c}28.00^{\prime \prime} \\
\text { to } \\
28.75^{\prime \prime}\end{array}$ & $28.75^{\prime \prime}$ \\
\hline \multirow{3}{*}{55 Gallon } & $\begin{array}{c}18 \\
\text { to } \\
20\end{array}$ & 18 & $22.50^{\prime \prime}$ & $22.50^{\prime \prime}$ & $\begin{array}{c}33.25^{\prime \prime} \\
\text { to } \\
34.75^{\prime \prime}\end{array}$ & $34.00^{\prime \prime}$ \\
\hline
\end{tabular}




\section{MATERIALS}

\section{1 $\mathrm{UO}_{2} \mathrm{~F}_{2}$-water solution vs. $\mathrm{UF}_{4}$-oil mixture}

Neither composition proved to be consistently more reactive than the other for all combinations of pitch and uranium density; thus $\mathrm{k}_{\mathrm{eff}}$ for both the $\mathrm{UO}_{2} \mathrm{~F}_{2}$-water solutions and $\mathrm{UF}_{4}$-oil mixtures were calculated. At uranium concentrations producing optimal reactivity (typically in the range between 1.5 and 3 grams of uranium per cc of solution) the $\mathrm{UF}_{4}$-oil mixture was the more reactive. Enrichments of both $2 \%$ and $5 \% 235 \mathrm{U}$ by weight were considered. Uranium concentrations of the materials were varied from 0.2 grams of uranium per cc of solution to the full theoretical density of the dry uranium compounds.

The composition of the $\mathrm{UO}_{2} \mathrm{~F}_{2}$-water mixture was derived from the density relationships in a recent ORNL publication [Ref. 5]. This treatment addresses the problem of experimental discrepancies found for uranium densities at very low $\mathrm{H} / \mathrm{U}$ ratio where hydrate compounds begin to form. The specification of UF4-oil mixture uses the KENO density multiplier option to specify its constituents. A description of these methods are found in Appendix 1.

\subsection{Concrete Composition}

The four concrete types contained in the KENO-V.a standard composition library were compared in a fixed array geometry. In contrast to the results from the geometry of the C.4 task involving the 42-unit storage tank arrays [Ref. 6], it was found that for this geometry, MG concrete was slightly more reactive than the other three choices. MG concrete was used for all subsequent calculations. See figures CON-1 and CON-2.

\subsection{Air/Water Mist Concentration}

The air in this array is modeled as water mist and occupies most of the volume in this calculation. The complete range of water mist concentration from full water flooding to extremely dry conditions were evaluated for its effect on the array reactivity. Calculation sets in which we varied the water mist density of the air surrounding the containers for several sets of pitch indicated little structure at low water densities but did indicate significant variation of $k_{\text {eff }}$ at water densities above $0.01 \mathrm{gm} / \mathrm{cc}$ which changed markedly for each of the calculated pitches. Since no mist value was consistently more reactive for all of the calculated pitches, we chose a moisture content of $10^{-4} \mathrm{gm}$ of water per cc of air. This value is representative of a normal humid environment, and was maintained throughout this study. See figures MD-1 to MD-4. 


\section{RESULTS}

\subsection{Pitch and Concentration Study-Normal Conditions}

The intent of this study was to investigate the effects of uranium concentration and pitch for infinite planar arrays of three commonly used waste receptacle sizes. The containers were filled with homogeneous mixtures of either $\mathrm{UO}_{2} \mathrm{~F}_{2}$-dissolved into water, or $\mathrm{UF}_{4}$ mixed with oil. Pitch was varied from essentially contact of the containers out to a distance where no significant change in reactivity was found by additional separation. The results of the completed calculations are seen in figures AA-AE through ZC-ZJ; tabular results are listed in Appendix 3. With the plot scaling we used, the error bars are contained within the plotting symbols and do not appear. All curves demonstrated the characteristic peak in reactivity near a uranium density of $2.0 \mathrm{gmU} / \mathrm{cc}$, falling off for both high and low concentrations. Discontinuities in the $\mathrm{k}_{\text {eff }}$ curves are evident for the $\mathrm{UO}_{2} \mathrm{~F}_{2}$-water mixtures at concentrations above $\sim 3.0 \mathrm{gmU} / \mathrm{cc}(\mathrm{H} / \mathrm{U}<4)$ due to hydrate formation.

\subsection{Extra Barrel-Accident Conditions}

Two types of accident case were considered; one in which an extra barrel is doublestacked on top of another, and one in which an extra barrel is inserted in between the rows and columns of the barrel array. Since neither of these accident cases can be represented by a uniform infinite array lattice, a new geometry model was coded around a baseline normal case containing a 21 by 21 square array of individual barrels. Cell spaces filled with air (low density water mist) were provided in between and on top of the barrels that could be interchanged for the extra barrel for the accident calculation. The four vertical edges at the border of the large array were specularly reflected to maintain the properties of an infinite array although the true lattice cell treatment was no longer applicable due to the perturbation that would be caused by the extra barrel. Half size spaces were used at the edges of the array and quarter size spaces were used at the four corners to maintain spacing uniformity upon reflection.

All container size and material variables are identical to the standard non-accident case. With this 21 by 21 geometry, we calculated a "baseline normal" case in which we use the new array but omit the extra barrel. With the appropriate effective pitch constructed with the use of air space cells, the "baseline normal" $\mathrm{k}_{\mathrm{eff}}$ agrees within $1 \%$ to the $\mathrm{k}_{\mathrm{eff}}$ calculated for the simple infinite square lattice array used for the standard normal case. 
Additional coding was provided in the KENO input to guarantee that neutrons were started in each of the 441 (442 for the accident case) fuel cells. When the spacing cells, between and on top of the fuel cells are included, the resultant array dimensions became 43 by 43 by 4. Figure 3 illustrates the normal and accident configuration of this array.

Three cases were chosen (one for each size barrel) to investigate the effects of the accidents. We chose cases that had just large enough pitch to allow an extra barrel to be interstitially inserted, and that had normal keff between 0.9 and 1.0. These cases were selected since they would be the most sensitive to array perturbations. All barrels contained $5 \%$ en riched $\mathrm{UO}_{2} \mathrm{~F}_{2}$-dissolved into water. The results are summarized below. As can be seen from the table, the effects on keff due to the accidents are barely above the one sigma statistical fluctuations; the worst offenders being the interstitial cases. At two sigma the accidents are insignificant.

All accident case calculations were run on the NCSS IBM-3090

\begin{tabular}{|c|c|c|c|}
\hline & \multicolumn{3}{|c|}{ Keffective \pm 1 sigma } \\
\hline case & normal baseline & interstitial accident & double stacked accident \\
\hline $\begin{array}{c}5 \text {-Gallon } \\
75 \mathrm{~cm} \text { pitch } \\
1.5 \mathrm{gmsU} / \mathrm{cc}\end{array}$ & $0.91644 \pm 0.00203$ & $0.92201 \pm 0.00201$ & $0.91836 \pm 0.00208$ \\
\hline $\begin{array}{c}30 \mathrm{Gallon} \\
100 \mathrm{~cm} \text { pitch } \\
0.4 \mathrm{gmsU} / \mathrm{cc}\end{array}$ & $0.93221 \pm 0.00169$ & $0.93766 \pm 0.00172$ & $0.93754 \pm 0.00178$ \\
\hline $\begin{array}{c}55 \mathrm{Gallon} \\
150 \mathrm{~cm} \text { pitch } \\
0.4 \mathrm{gmsU} / \mathrm{cc}\end{array}$ & $0.96845 \pm 0.00232$ & $0.97588 \pm 0.00242$ & $0.96877 \pm 0.00221$ \\
\hline
\end{tabular}

\section{CONCLUSIONS}

The comments below are generalizations. For precise data refer to the applicable graphs and tables appearing later in this article.

\subsection{Gallon Drums}

This is the only size barrel that could be spaced far enough apart to remain subcritical at all uranium densities. At $5 \%$ enrichment, square pitch spacing could be as close as $100 \mathrm{~cm}$ for the most reactive $U_{4}$-oil mixture and $75 \mathrm{~cm}$ for the most reactive $\mathrm{UO}_{2} \mathrm{~F}_{2}$-water mixture. At $2 \%$ enrichment, the spacing for both materials could be safely reduced to $50 \mathrm{~cm}$ for all uranium densities. 


\subsection{Gallon Drums}

At $5 \%$ enrichment only uranium densities below $\sim 0.3 \mathrm{gmU} / \mathrm{cc}$ or above $\sim 4.5 \mathrm{gmU} / \mathrm{cc}$ were subcritical at all values of pitch. At $2 \%$ the lower limit could be raised to $\sim 1.0 \mathrm{gmU} / \mathrm{cc}$. At the greatest separation considered $(200 \mathrm{~cm})$, the subcritical density could be raised to $\sim 0.45 \mathrm{gmsU} / \mathrm{cc}$ for the $5 \%$ case and any density for the $2 \%$ case.

\subsection{Gallon Drums}

The 55 gallon results are quite similar to the 30 gallon case. At $5 \%$ enrichment only uranium densities below $\sim 0.3 \mathrm{gmU} / \mathrm{cc}$ or above $\sim 4.5 \mathrm{gmU} / \mathrm{cc}$ were subcritical at all values of pitch. At $2 \%$ the lower limit could be raised to $\sim 1.0 \mathrm{gmU} / \mathrm{cc}$. At the greatest separation considered $(500 \mathrm{~cm})$, the subcritical density could be raised to $\sim 0.4 \mathrm{gmsU} / \mathrm{cc}$ for the $5 \%$ case but not above $1.0 \mathrm{gmU} / \mathrm{cc}$ for the $2 \%$ case.

\section{REFERENCES}

1. SCALE: A Modular Code System of Performing Standardized Computer Analysis for Licensing Evaluation, NUREG/CR-0200, Revision 4 (ORNL/NUREG/CSD-2/R4), Vols. I, II, and III (April, 1991). Available from Radiation Shielding Information Center as CCC-545

2. D.W. Nigg, C.A. Atkinson, J. Blair Briggs, J. Todd Taylor, "A PC-Based Version of Keno 5.a," Trans. Am. Nucl. Soc. 61, 188 (1990)

\section{ANSI/ASC MH2-1985:}

Section 7.1 American National Standard for 5-Gallon (19-Liter) Tight-Head Steel (Double-Seam Chime) Pails (DOT-17E, DOT-37B60, UFC-Rule 40, NMFC-Item 260)

Section 4.3 American National Standard for 30-Gallon (114-Liter) Full-RemovableHead Steel (Double-Seam Chime) Drums (DOT-17H, UFC-Rule 40, NMFC-Item 260)

Section 3.1 American National Standard for 55-Gallon (208-Liter) Tight-Head Steel (Double-Seam Chime) Drums (DOT-17E, UFC-Rule 40, NMFC-Item 260)

4. Telephone conversation between Howard Dyer and Bill Lloyd on July 29, 1992

5. W.C. Jordan and J.C. Turner, "Minimum Mass of Moderator Required for Criticality of Homogeneous Low-Enriched Uranium Systems," ORNL/CSD/TM-284, Martin Marietta Energy Systems, Inc., Oak Ridge National Laboratory, December 1992.

6. W.R. LLoyd and B.A. Pohl, "K-effective Evaluation of the C-400/409 Acidification and Storage Tank Arrays at the Paducah Gaseous Diffusion Plant," UCRL-ID-112338, January 29,1993 


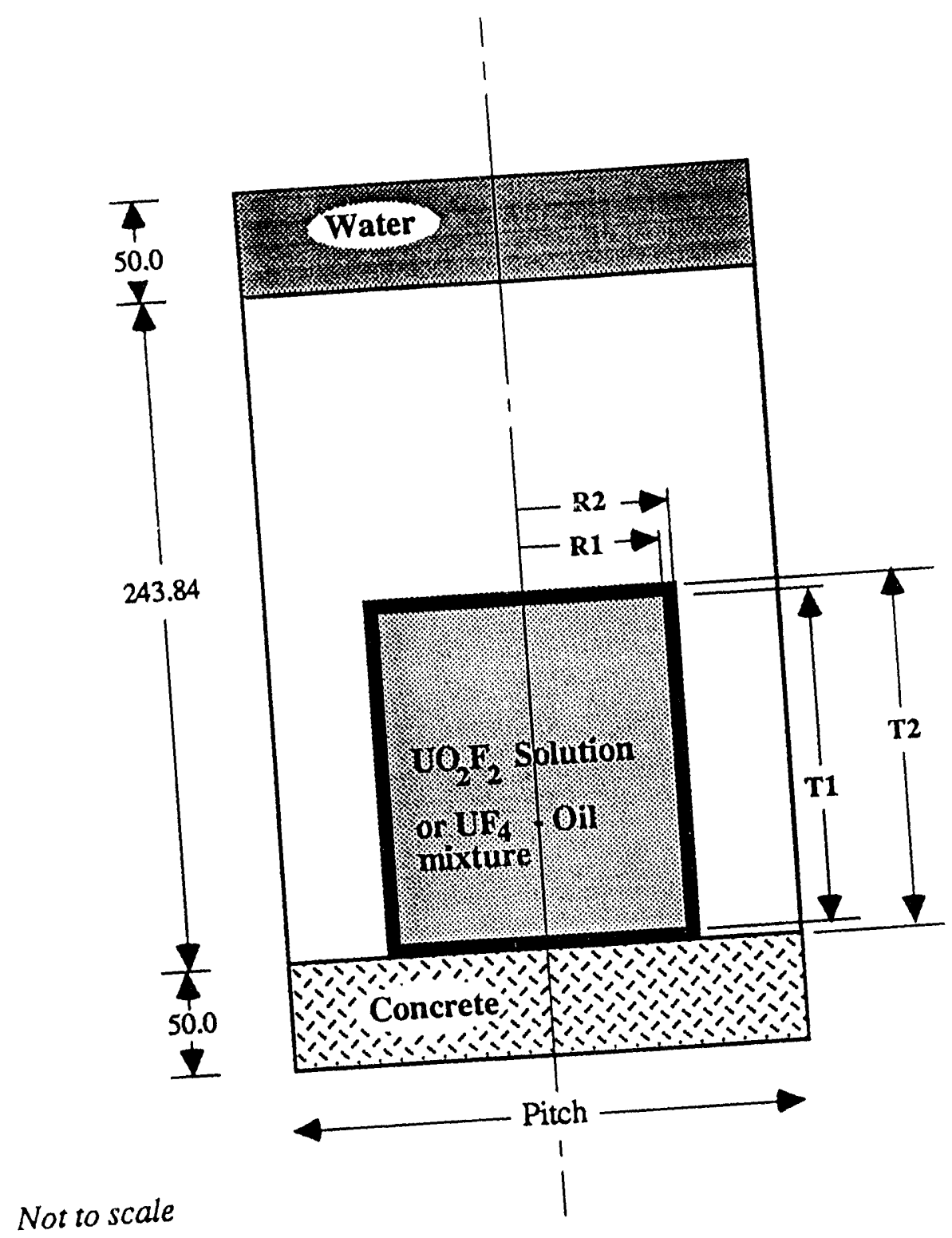

Not to scale

\begin{tabular}{|c|c|c|c|c|}
\hline \multirow{2}{*}{$\begin{array}{c}\text { Container Volume } \\
\text { (gal) }\end{array}$} & $\mathbf{4} 1$ & $\mathbf{R 2}$ & $\mathbf{T 1}$ & Dimension $(\mathrm{cm})$ \\
\hline \hline 5 & 14.2875 & 14.3202 & 34.1313 & 34.1968 \\
30 & 23.1775 & 23.2862 & 73.025 & 73.2424 \\
55 & 28.575 & 28.684 & 86.36 & 86.577 \\
\hline
\end{tabular}

Figure 1 
Task C-3 KENO RUNS

Labeling Convention for Calculation Sets

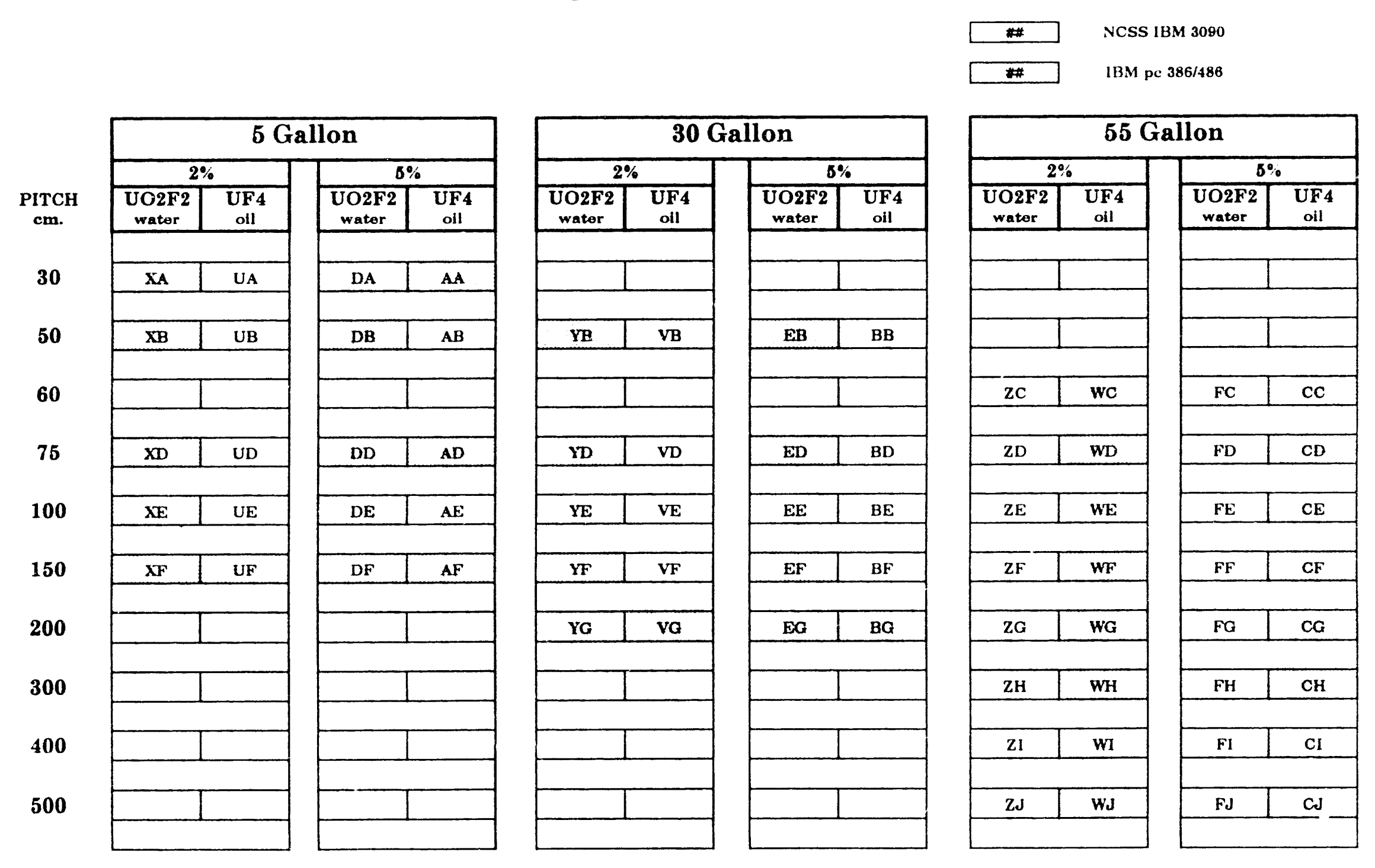

Figure 2 
Array Structure for the Accident Cases

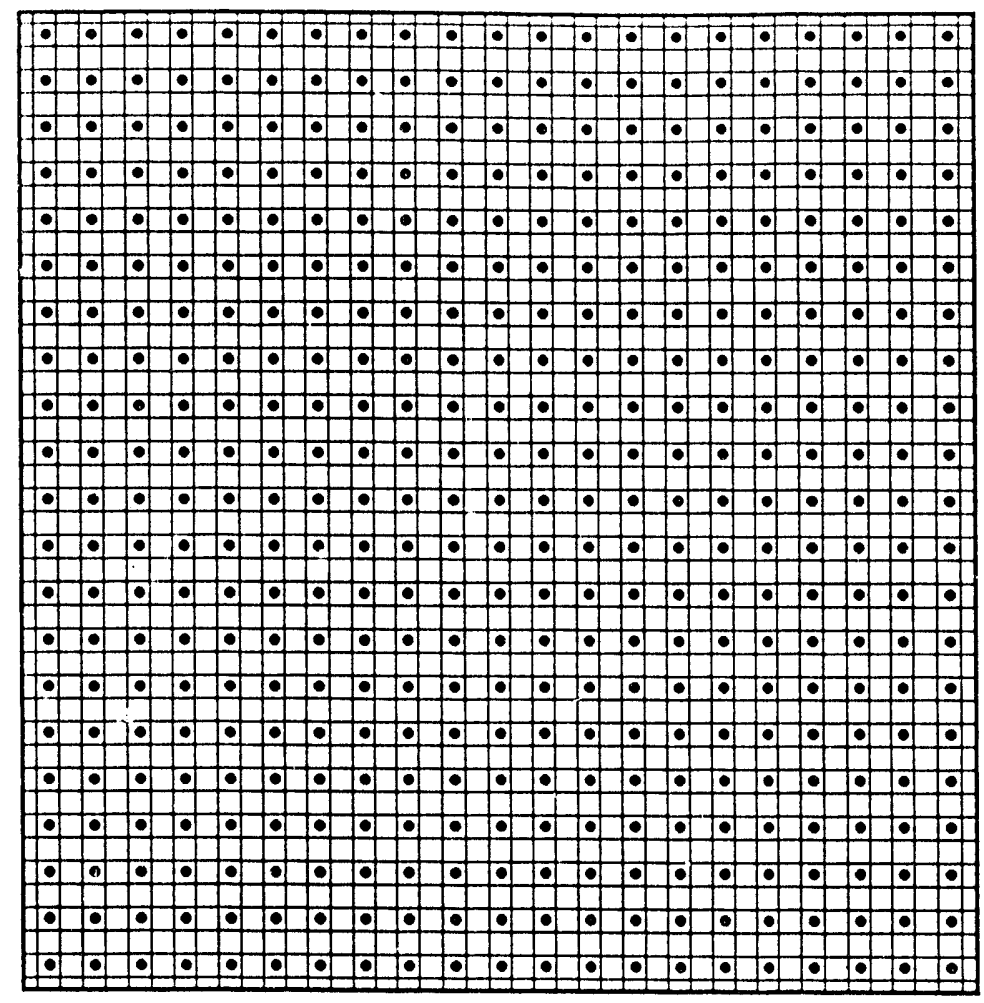

Uniform Array - Normal Condition

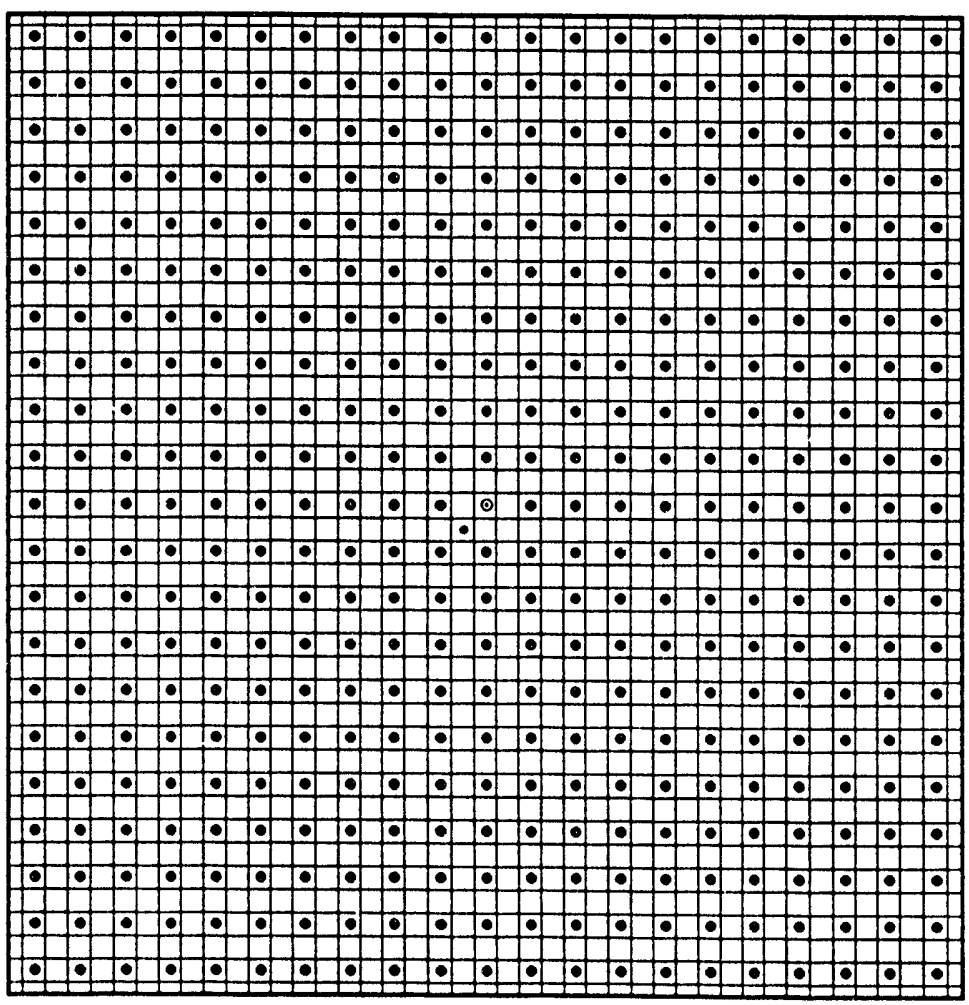

- Location for the Extra Interstitial Container

- Location for the Extra Double Stacked Container

Figure 3 
Graphical Results 


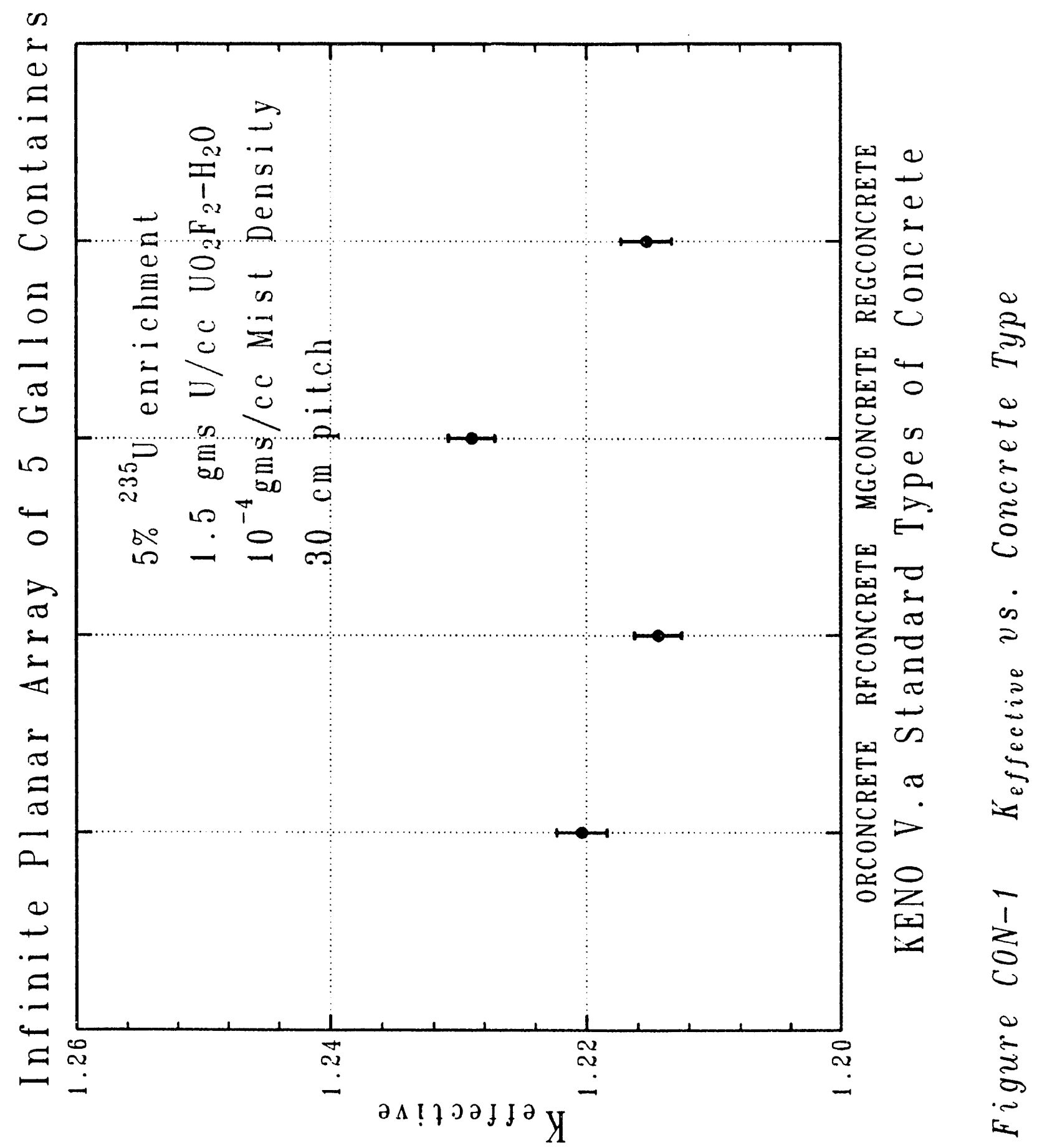




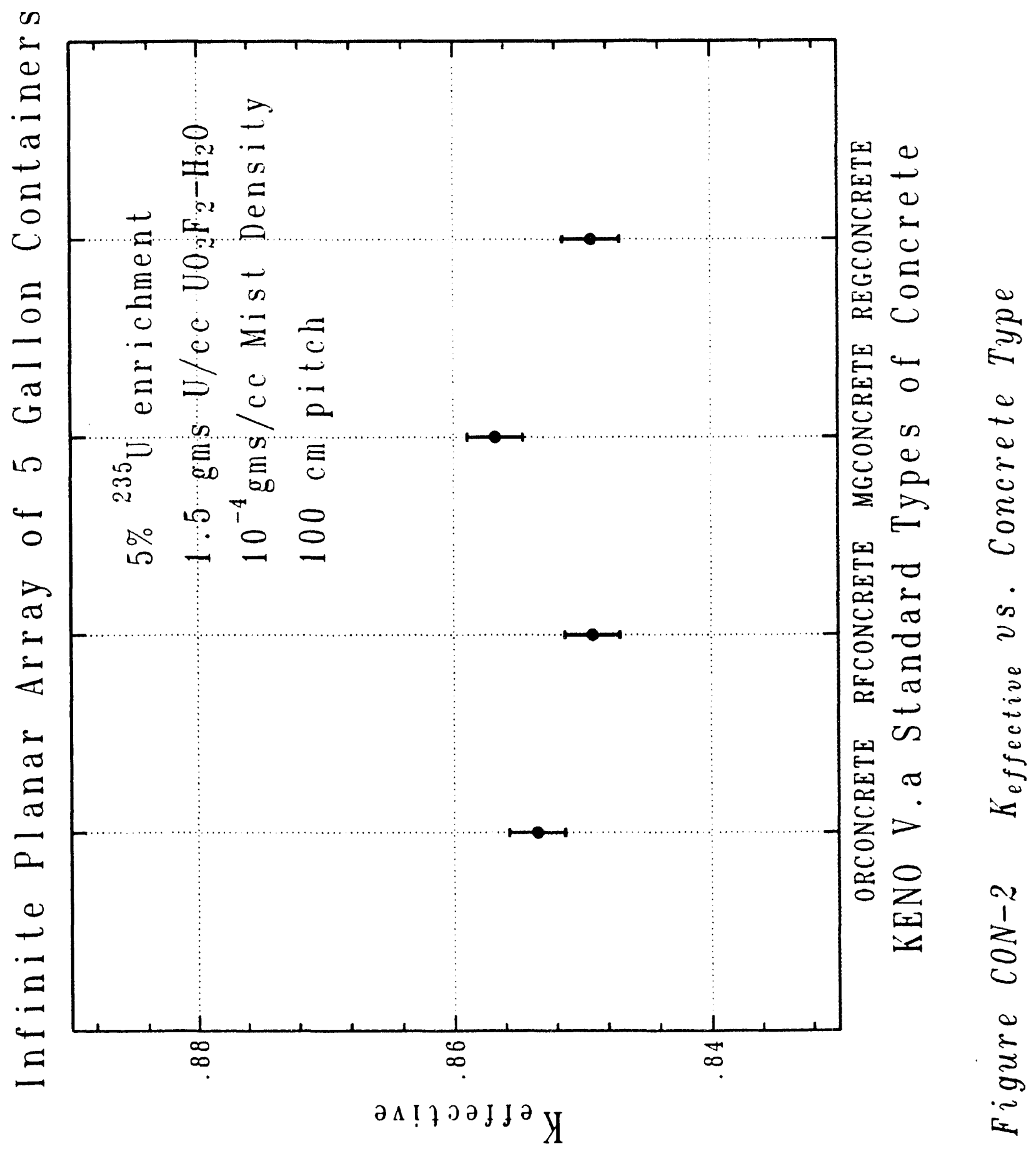




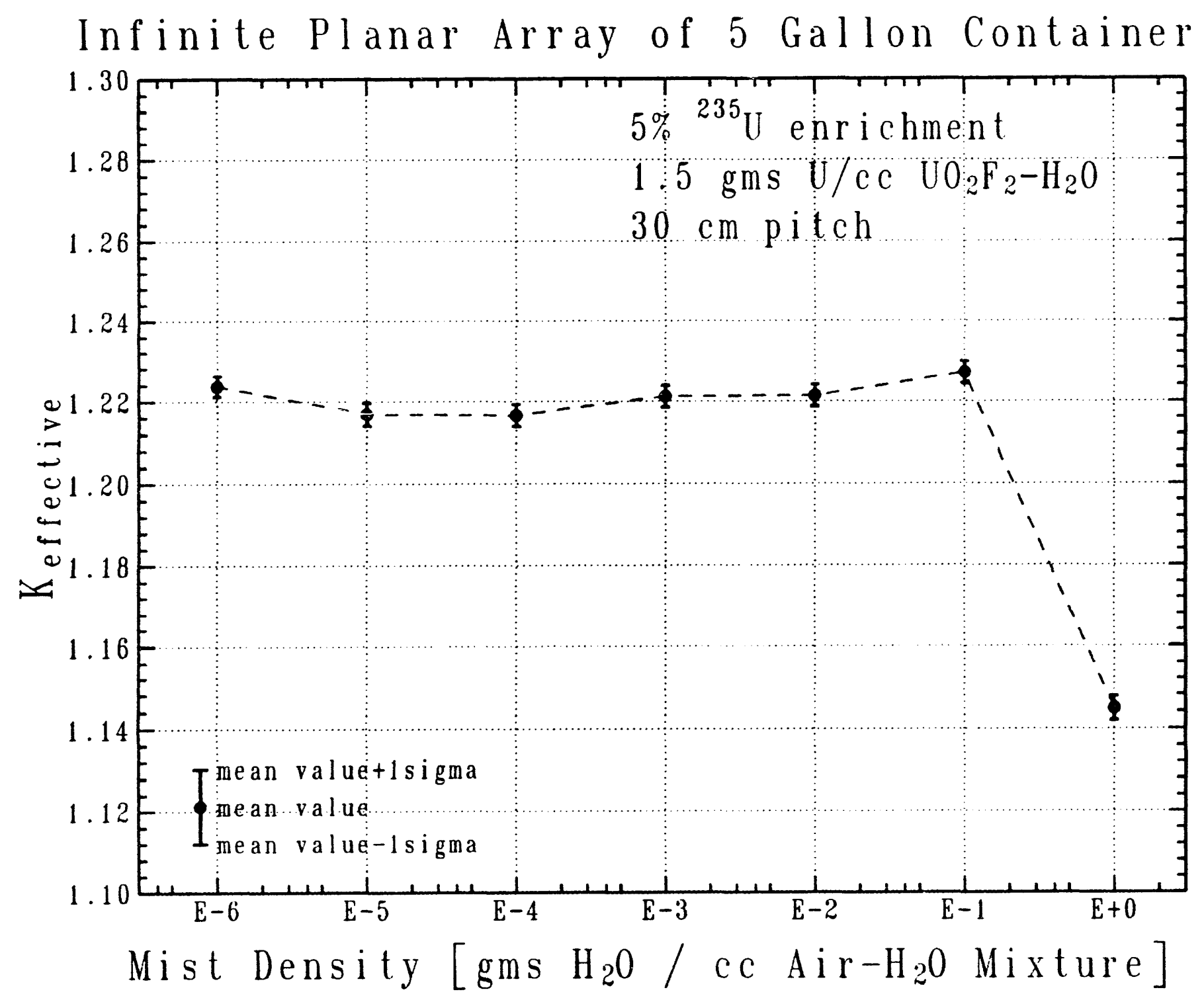

Figure MD-1 Keffective vs. Mist Density 


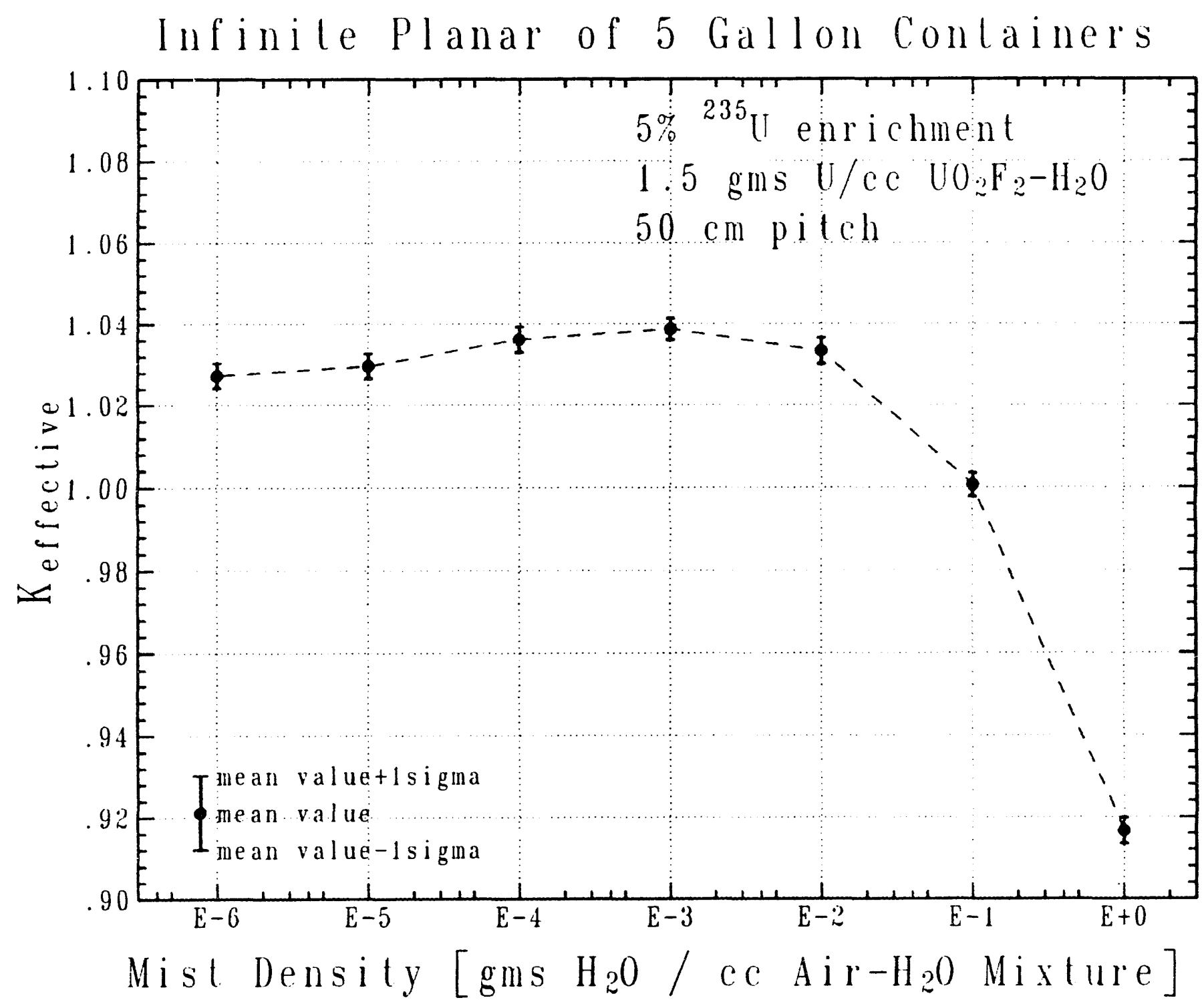

Figure MD-2 Keffective vs. Mist Density 


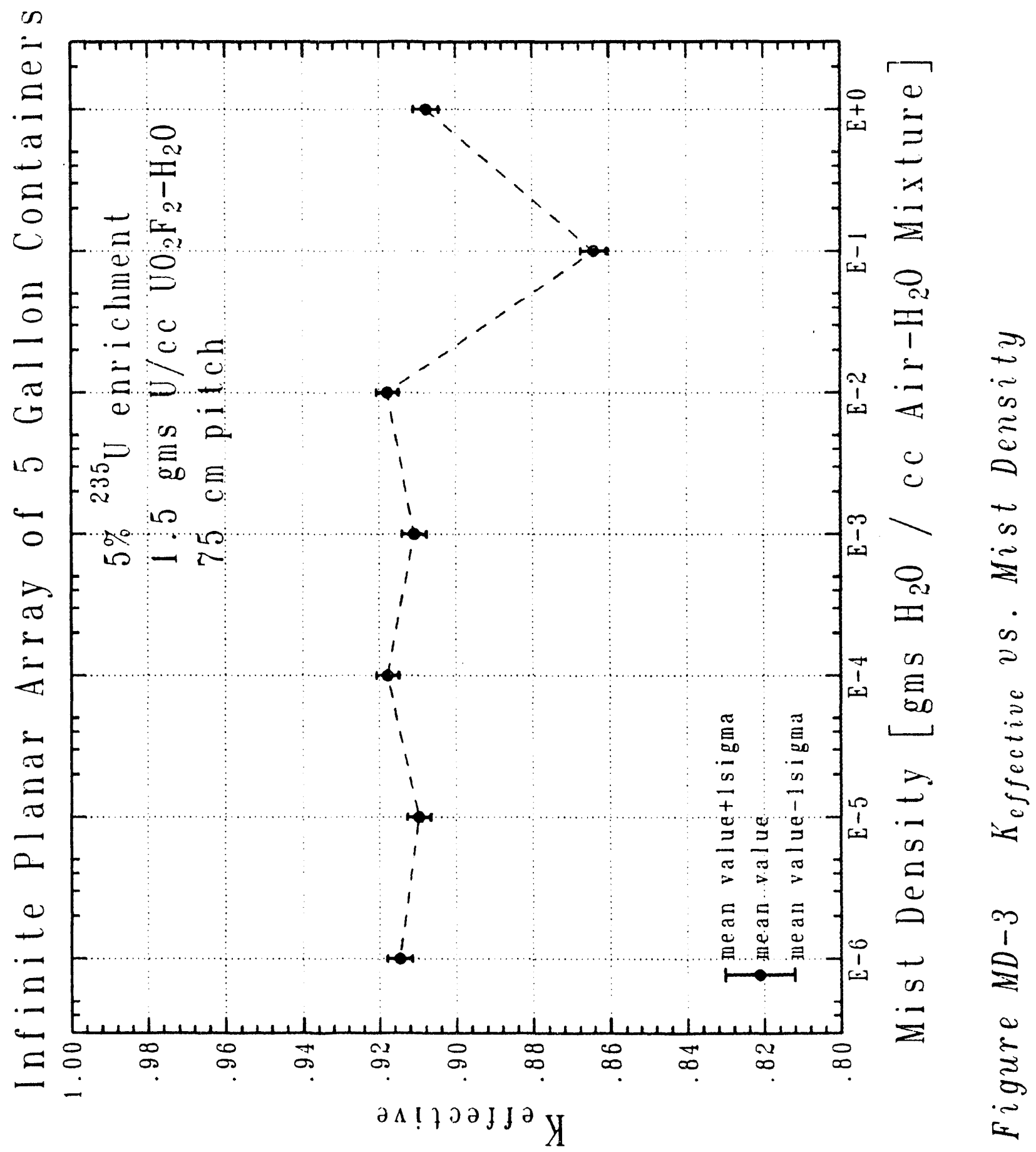




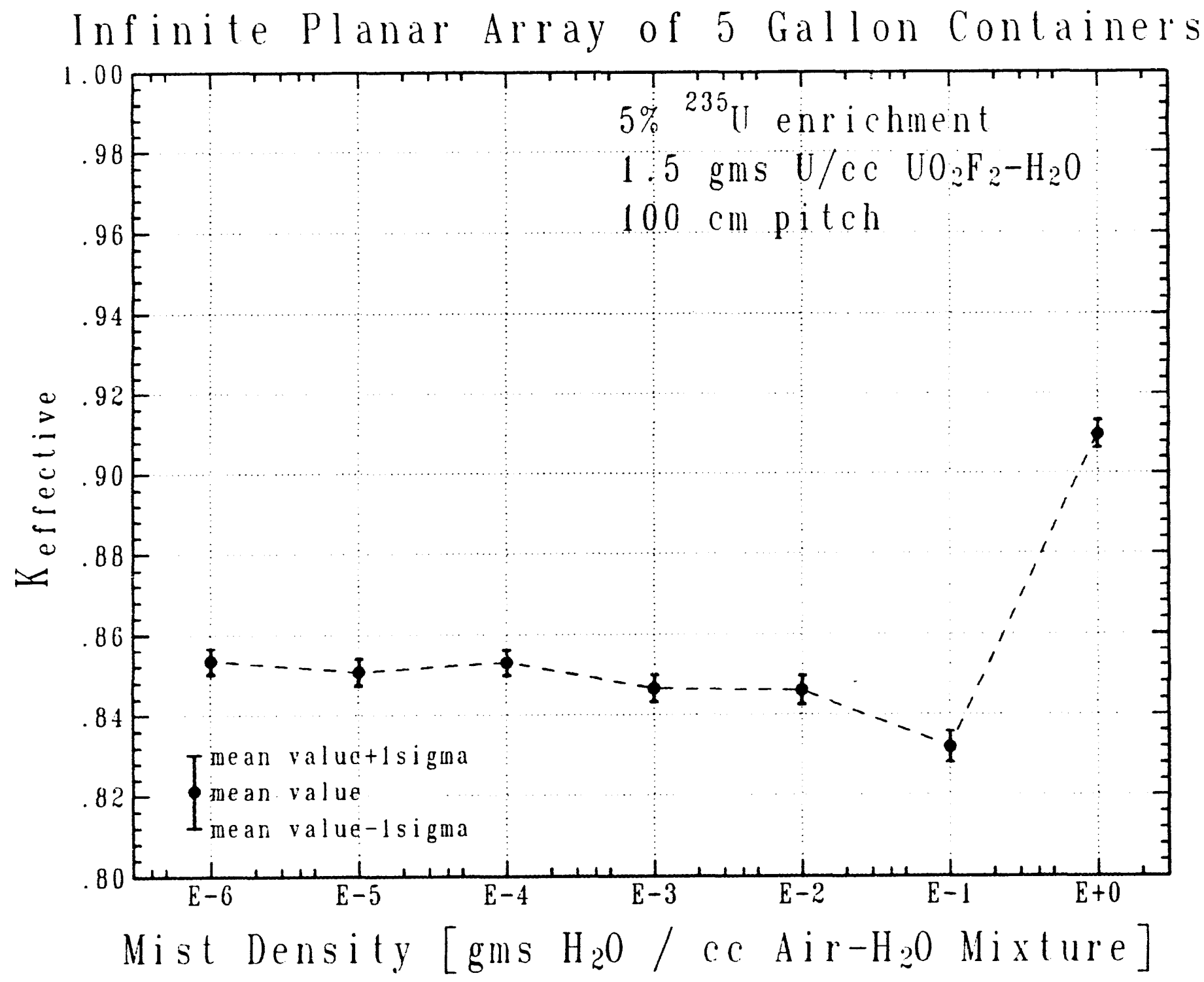

Figure MD-4 $K_{\text {effective }}$ vs. Mist Density 


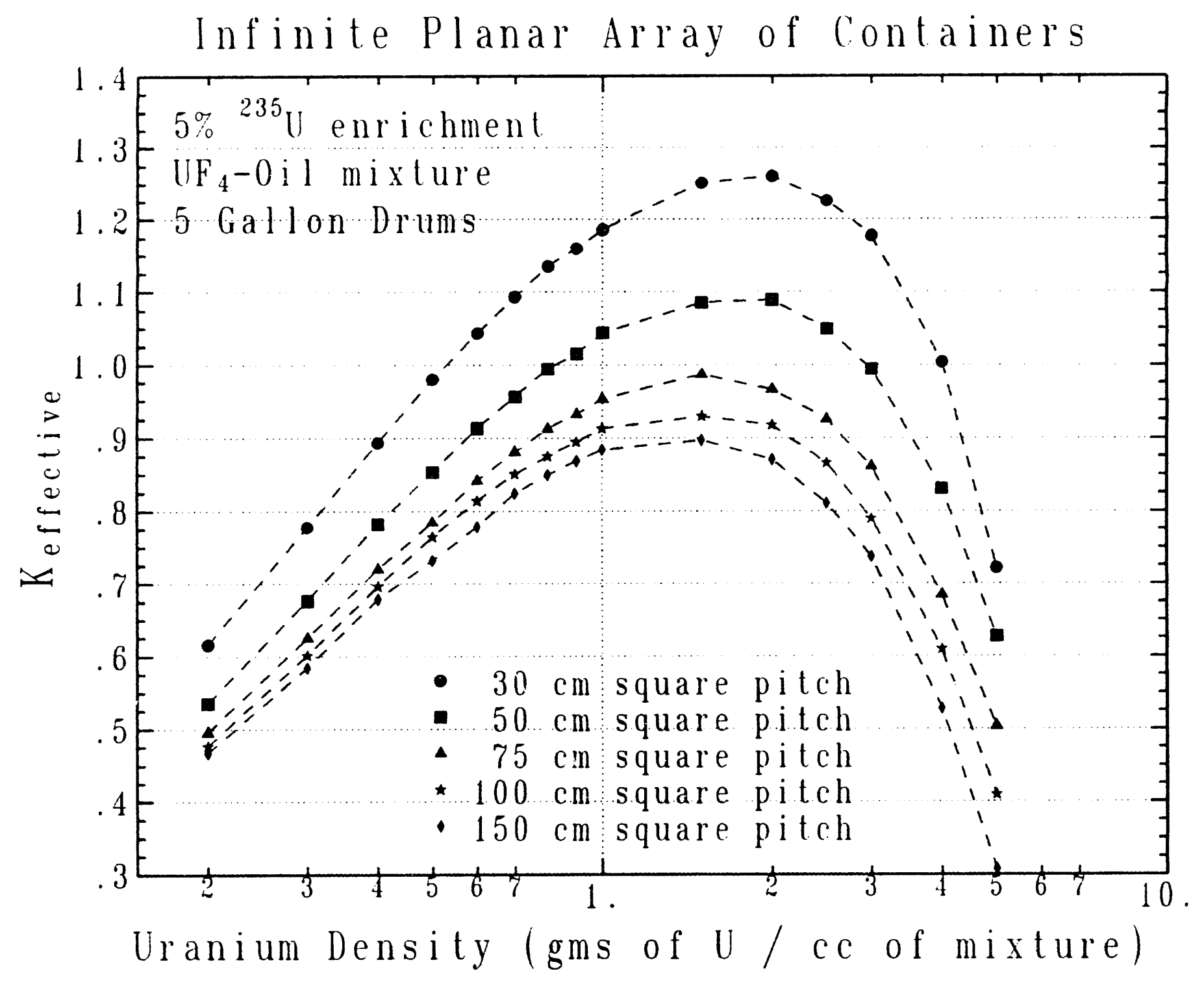

Figure $A A-A F \quad K_{\text {effective }} v s$. Uranium Density 


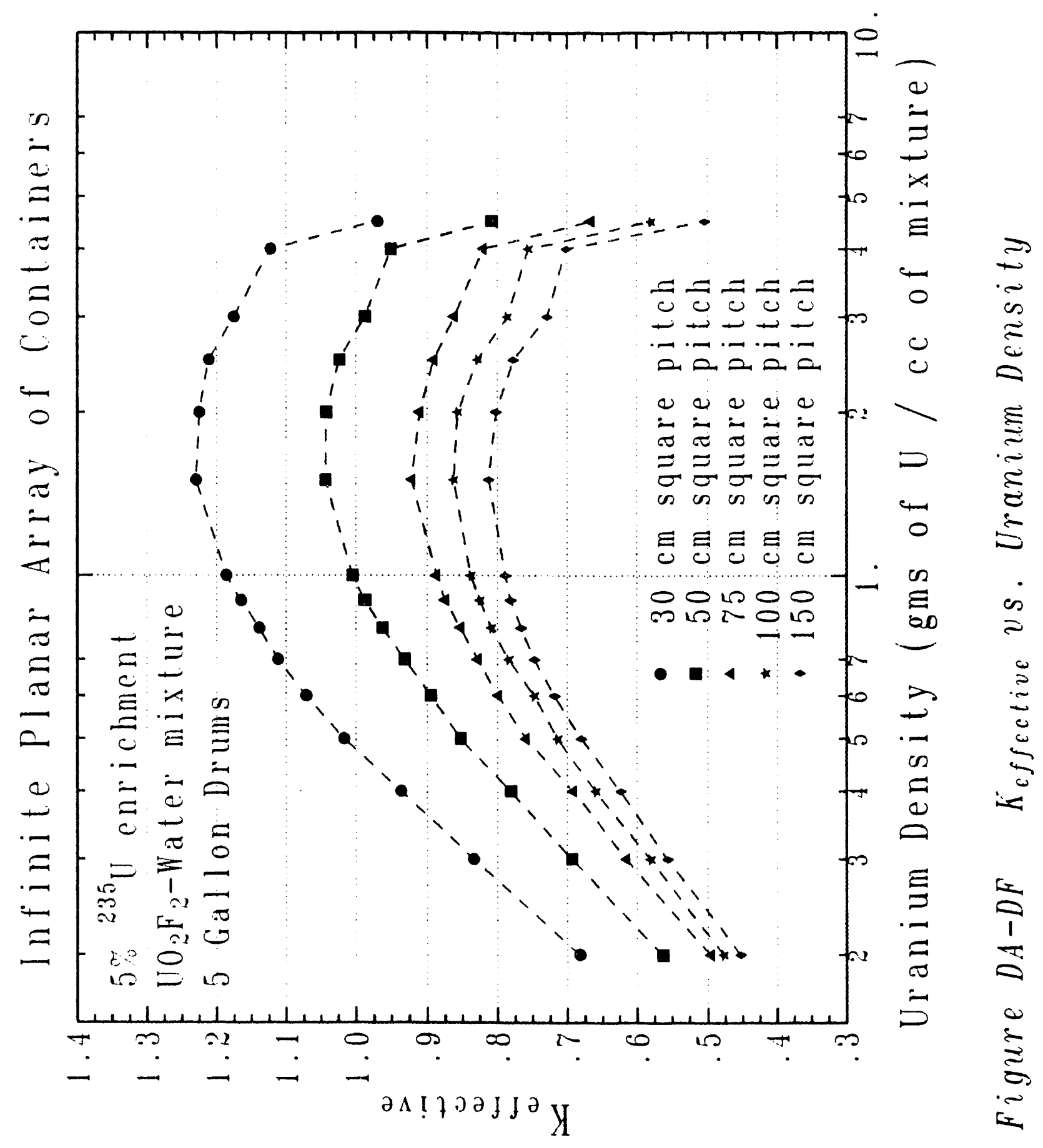




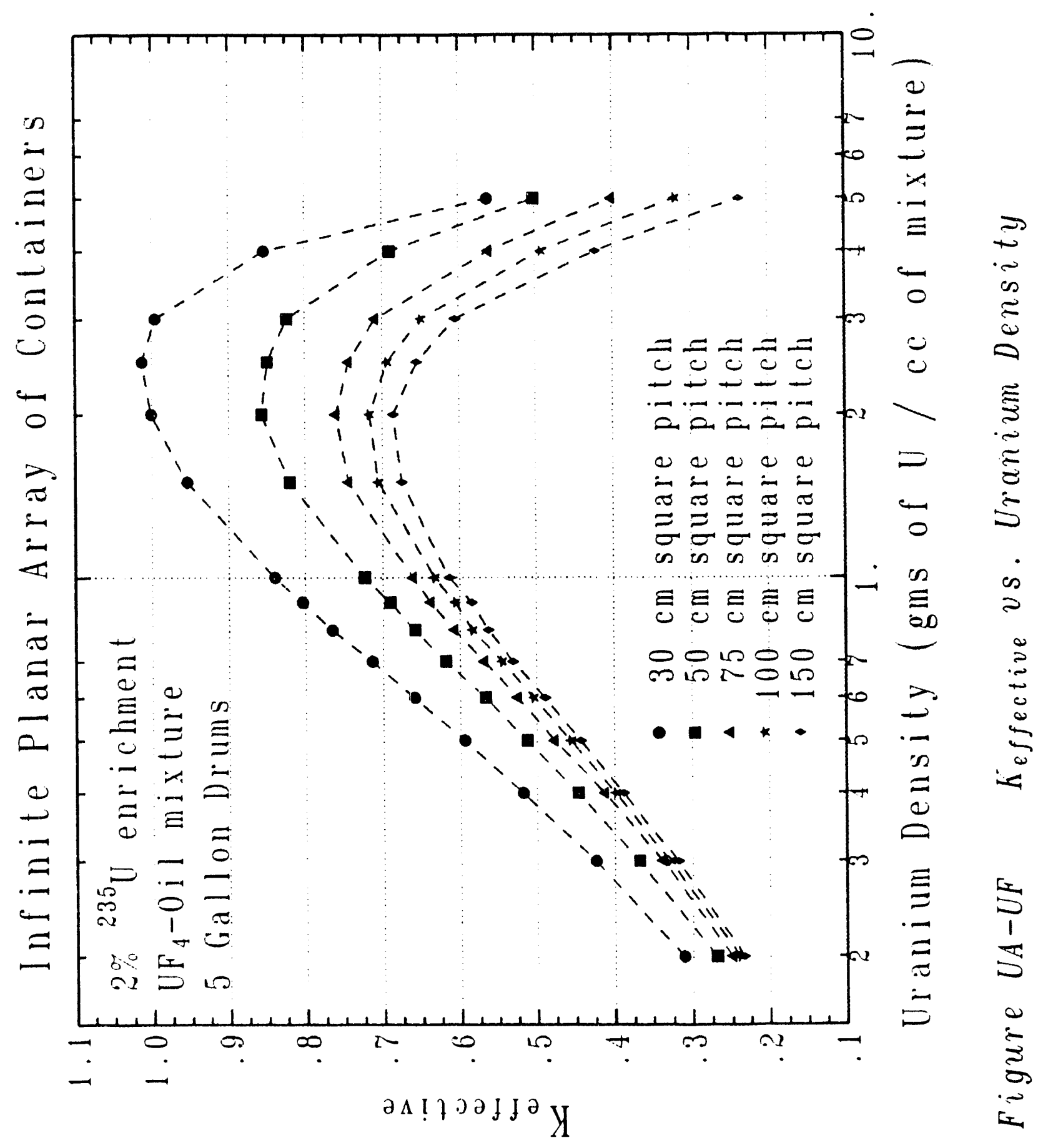


Infinite Planar Array of Containers

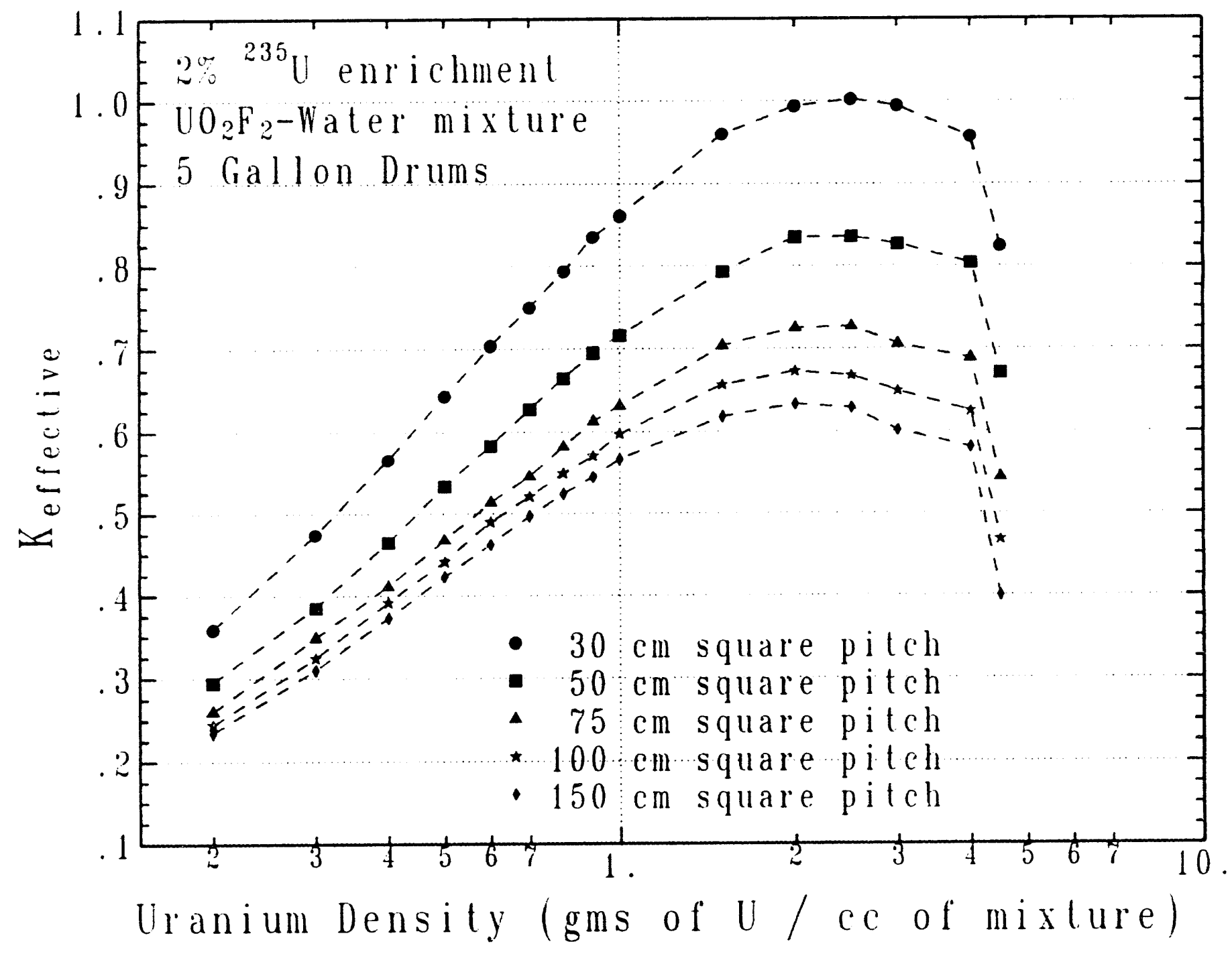

Figure XA-XF $K_{\text {effective }} v s$. Uranium Density 


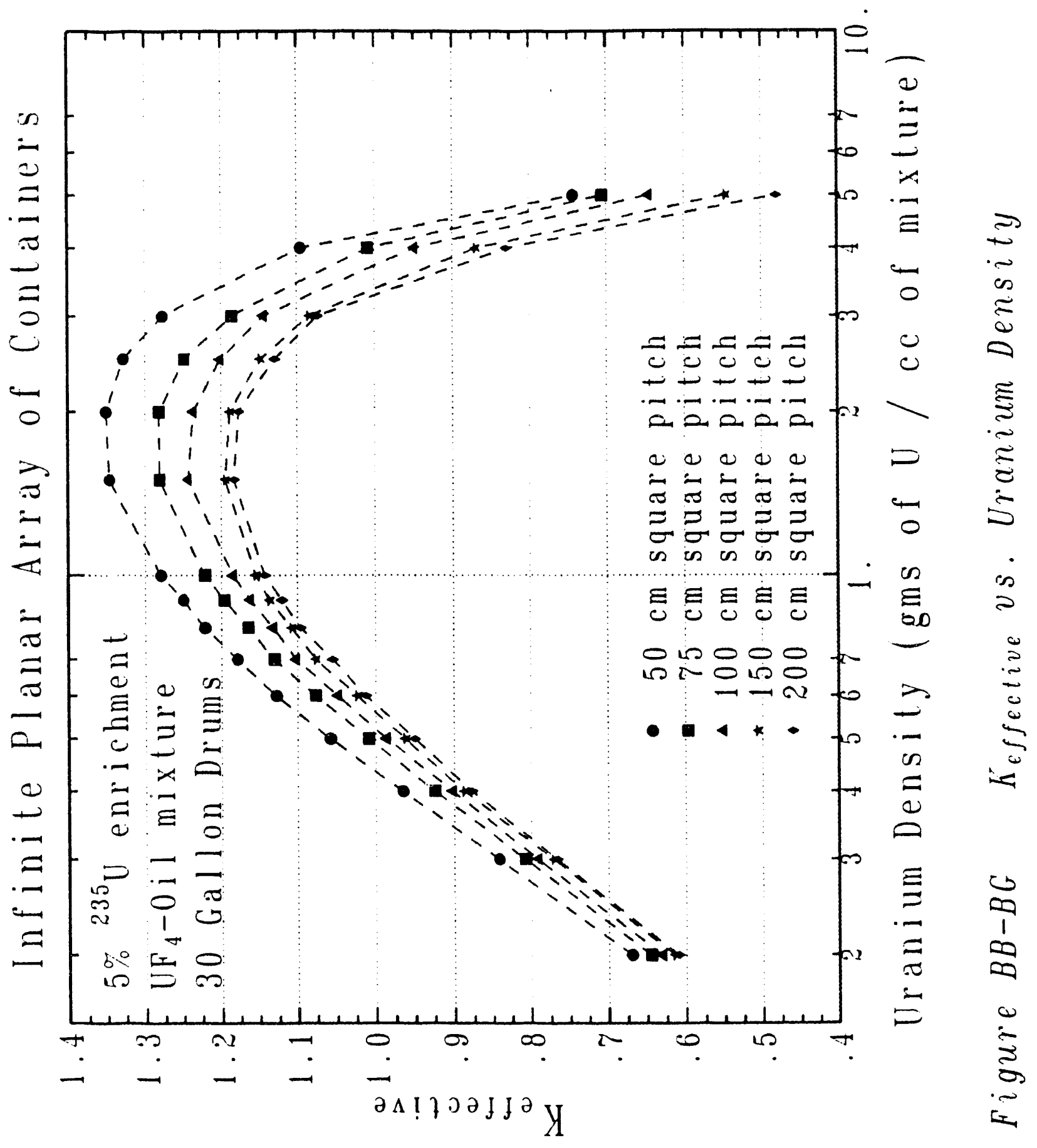




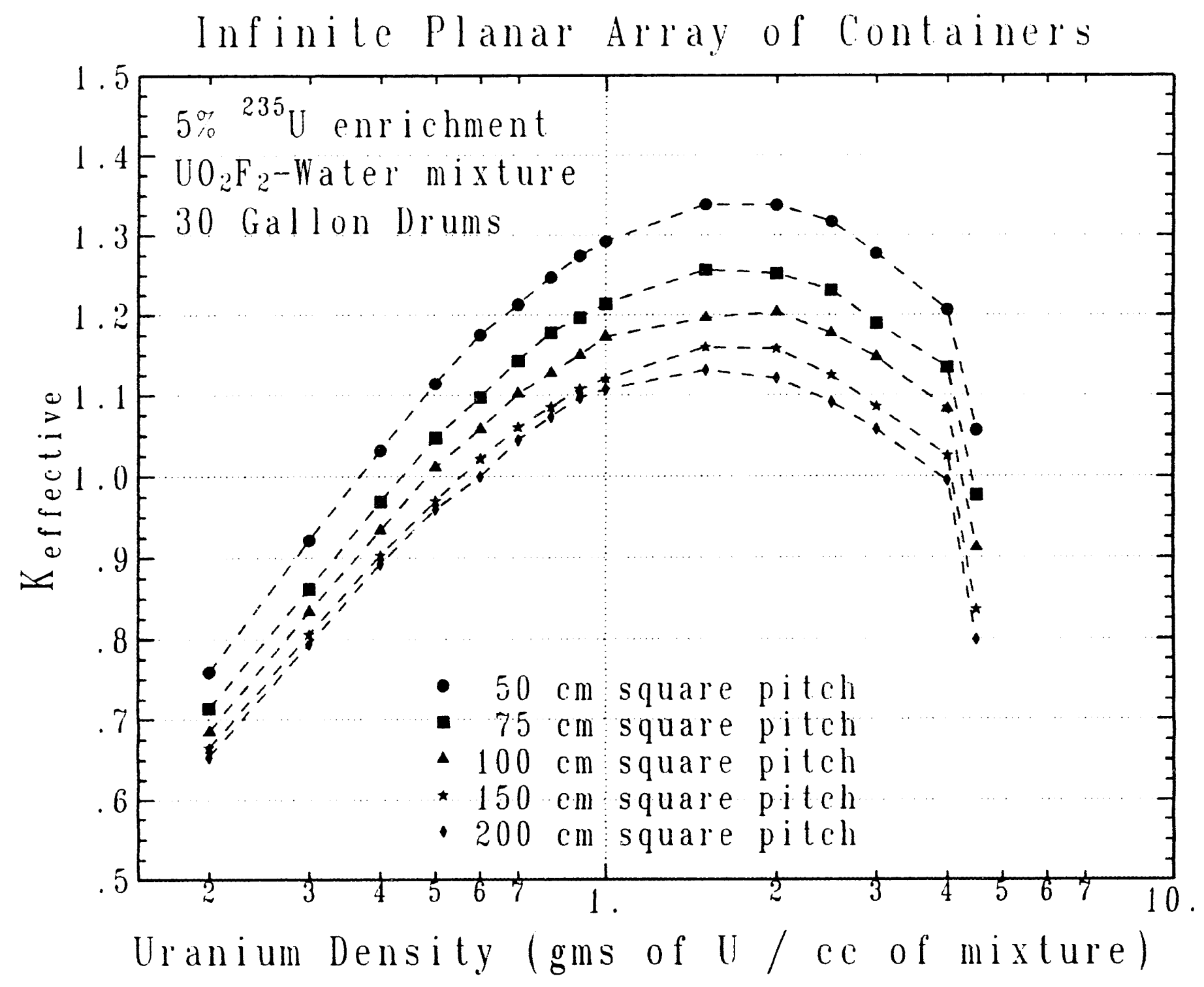

Figure EB-EG Keffective $v s$. Uranium Density 


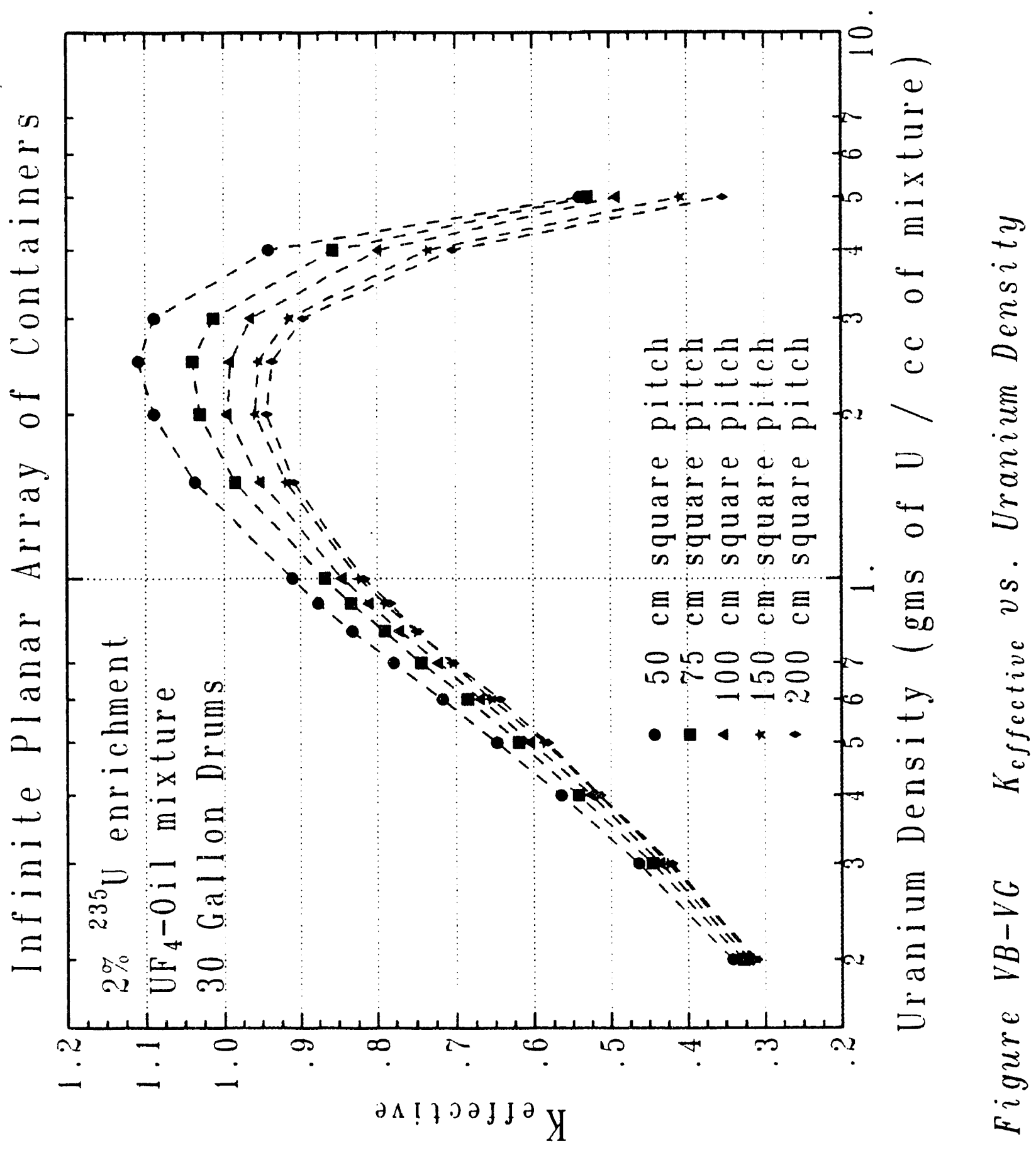




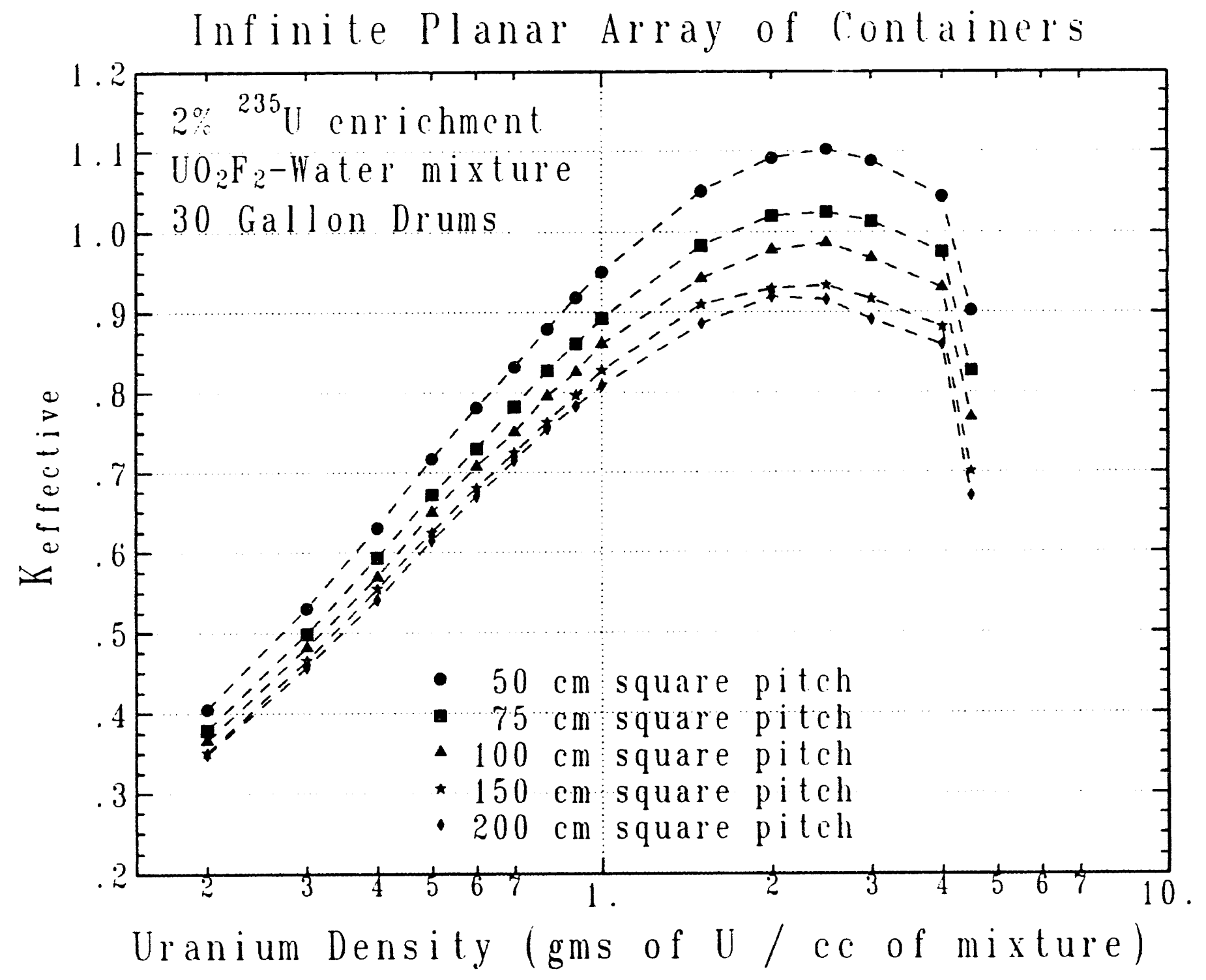

Figure $Y B-Y G \quad K_{\text {effective }} v s$. Uranium Density 


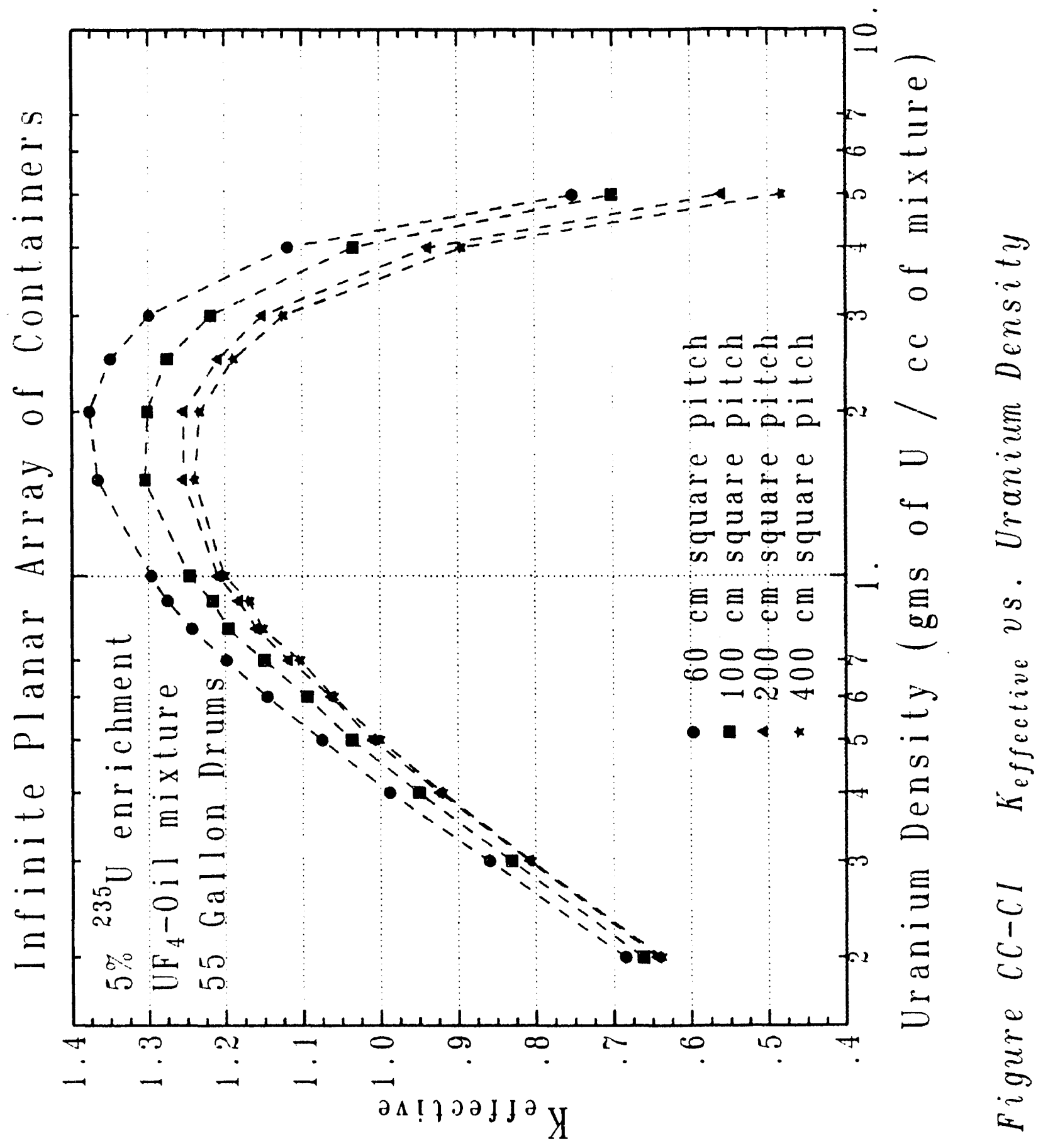




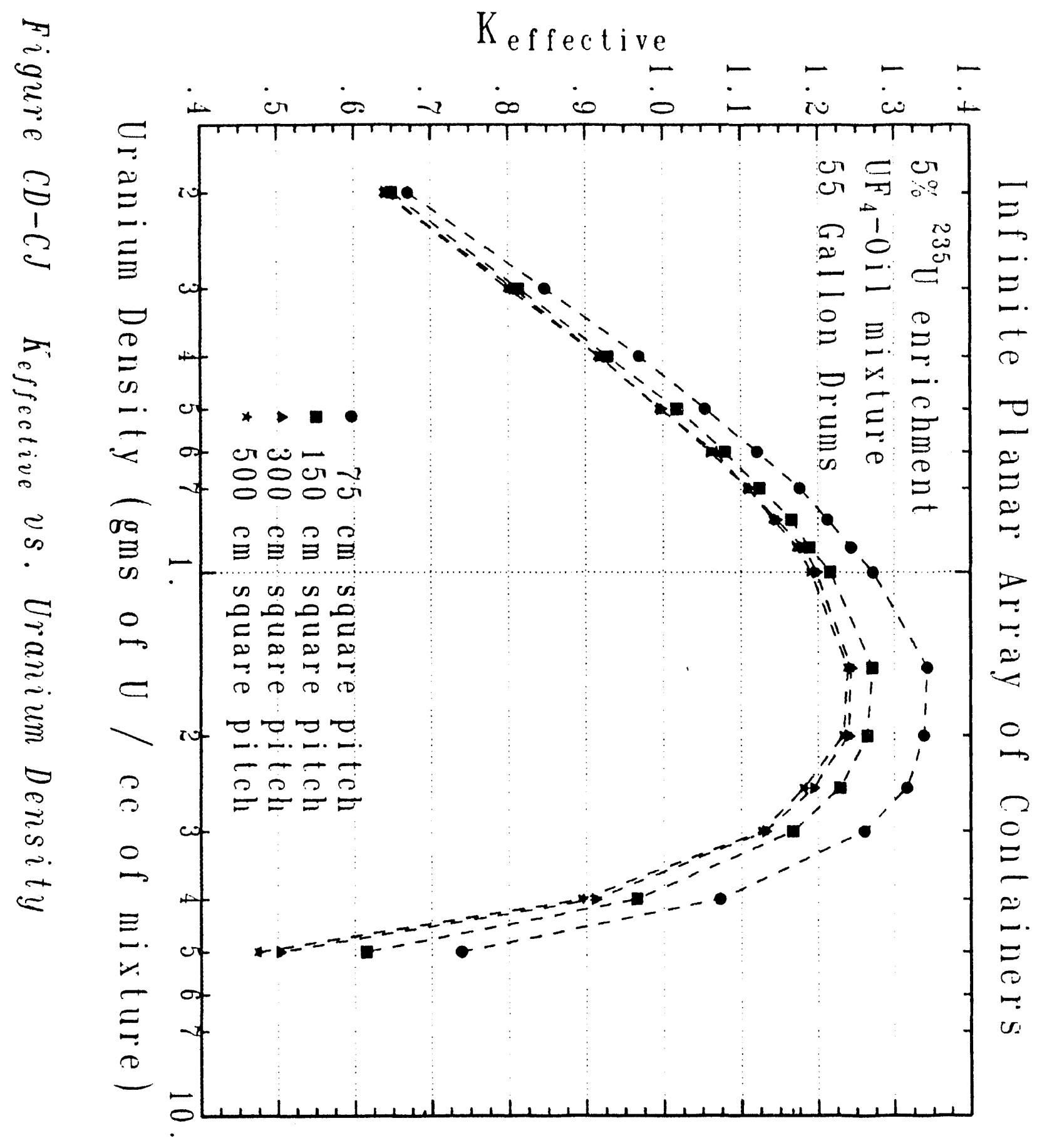




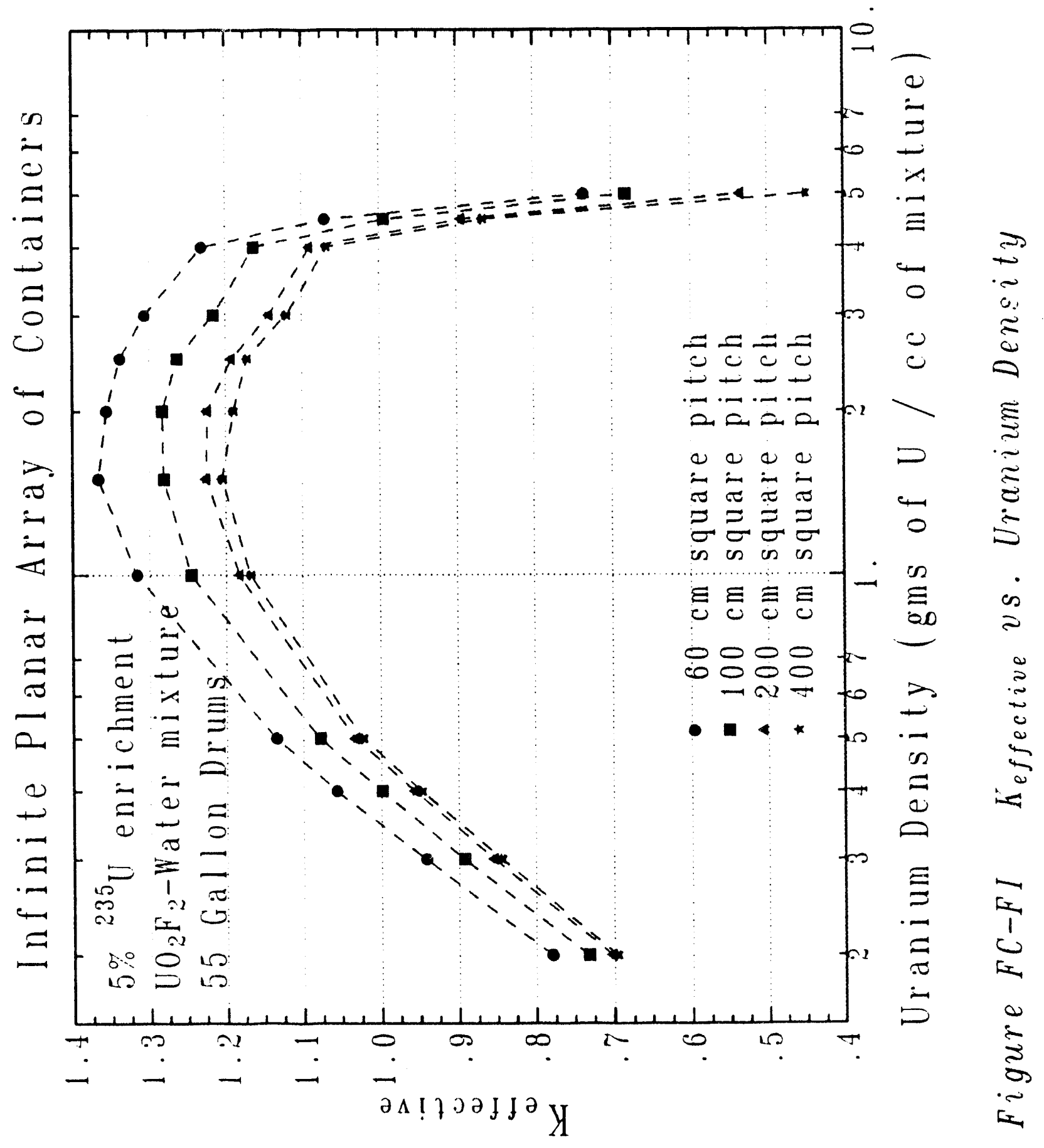




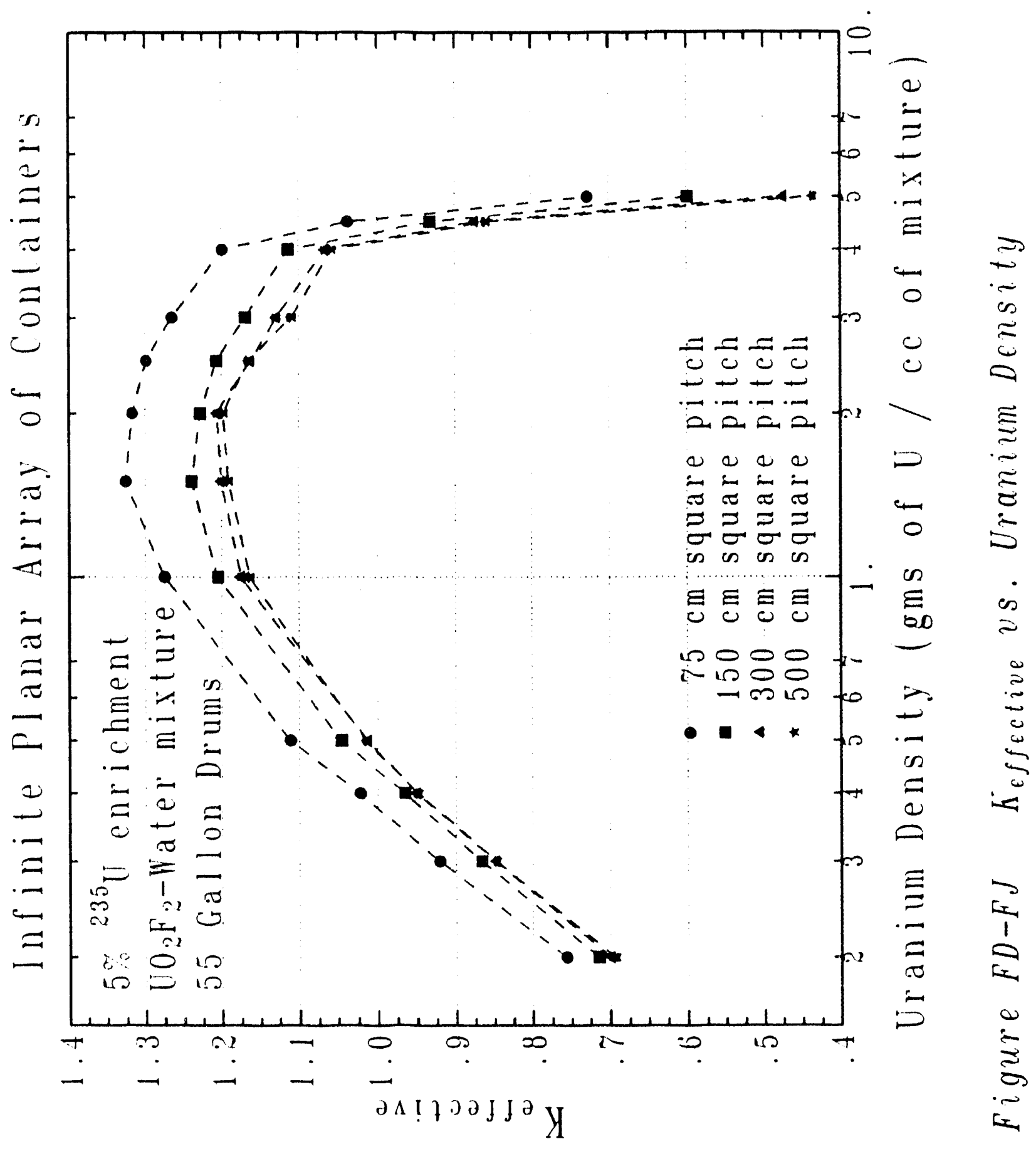




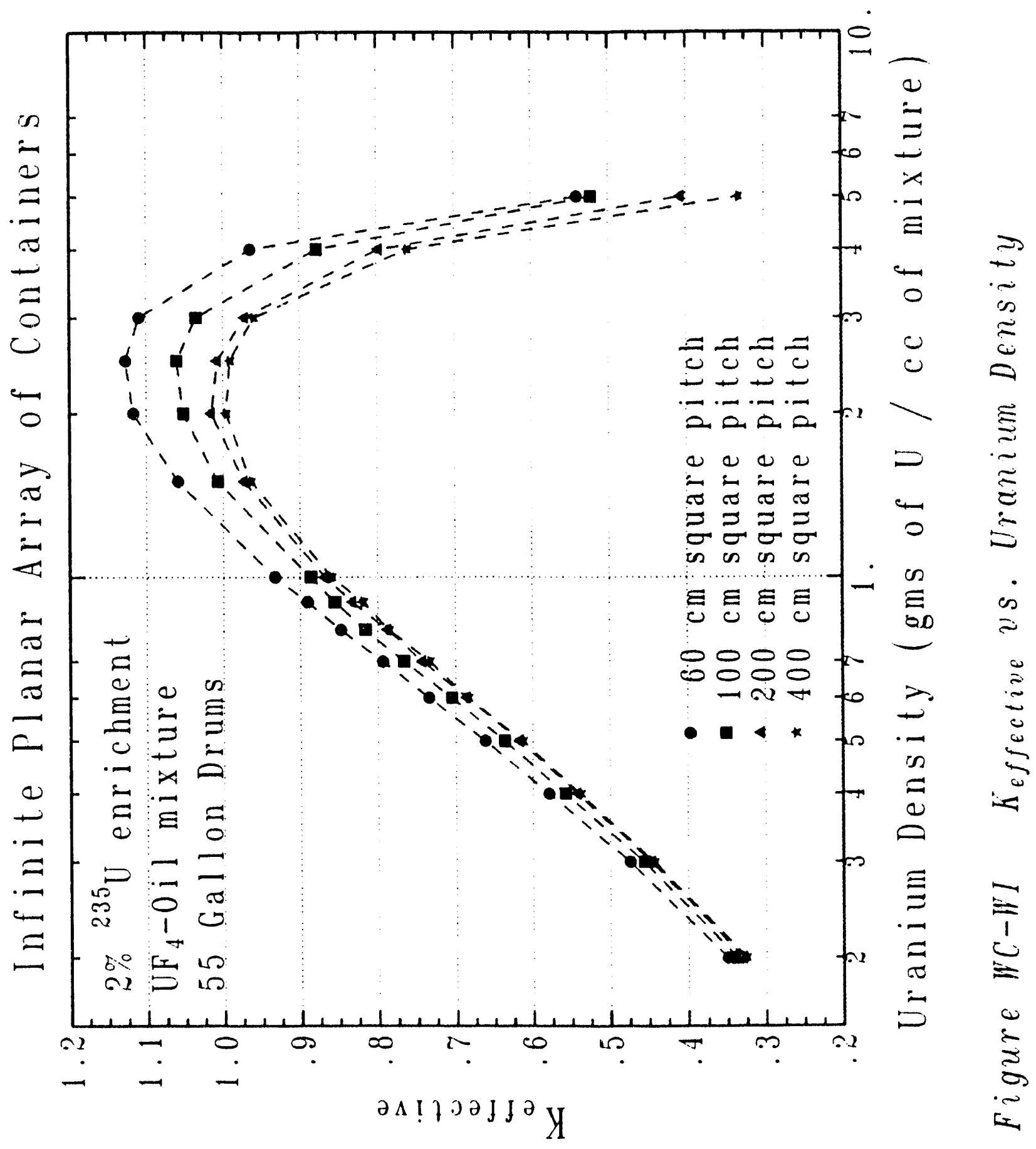




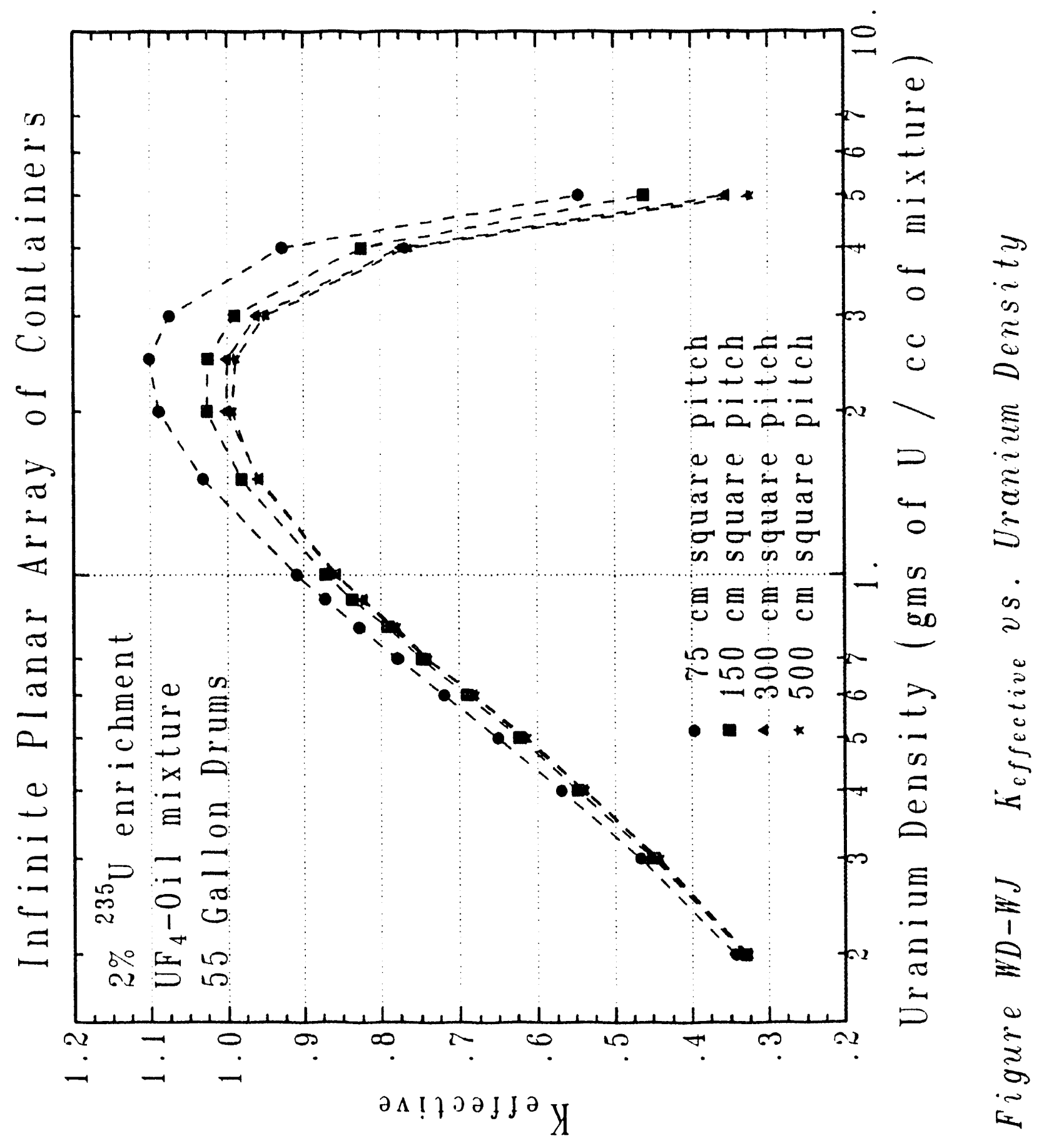




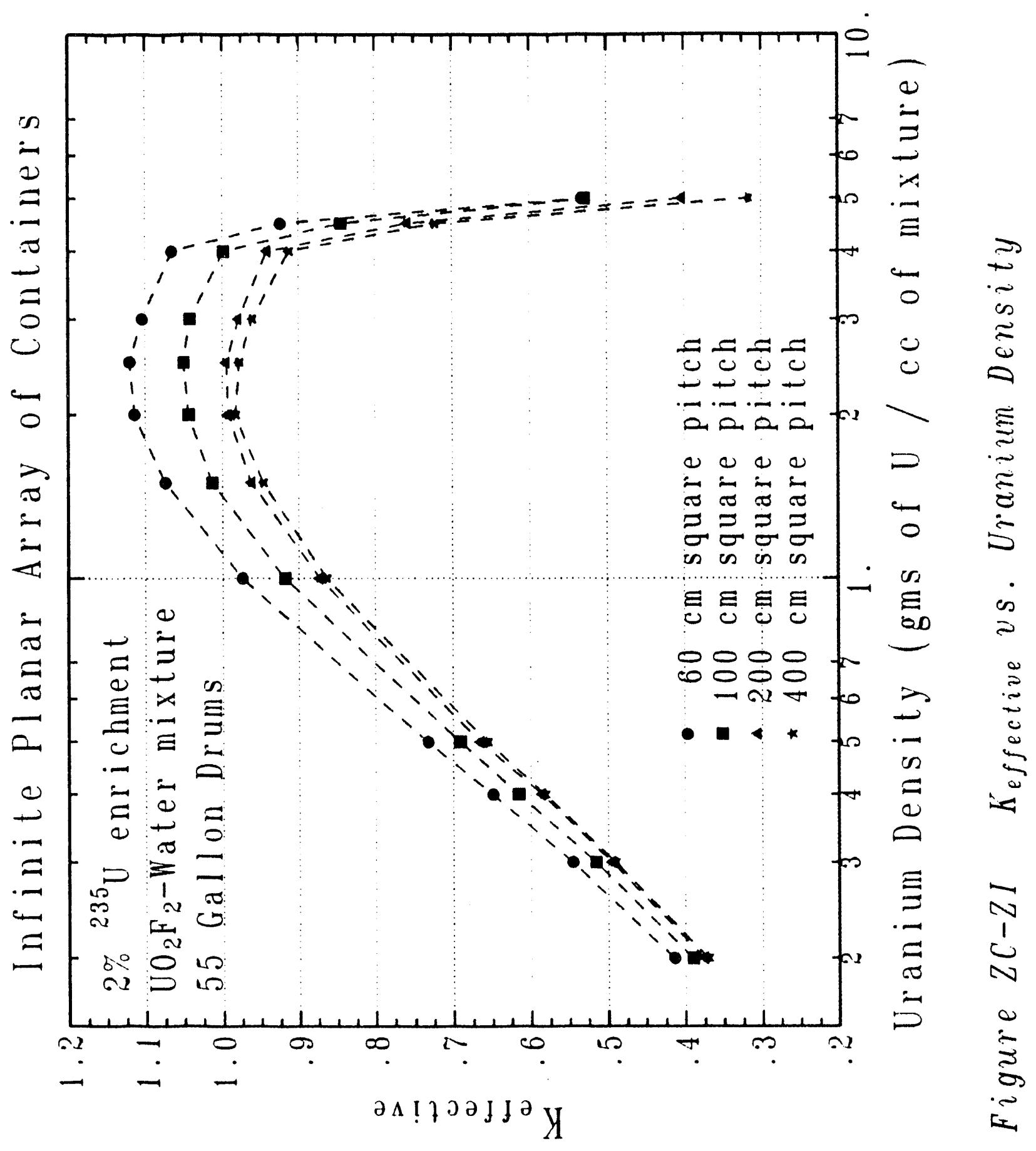




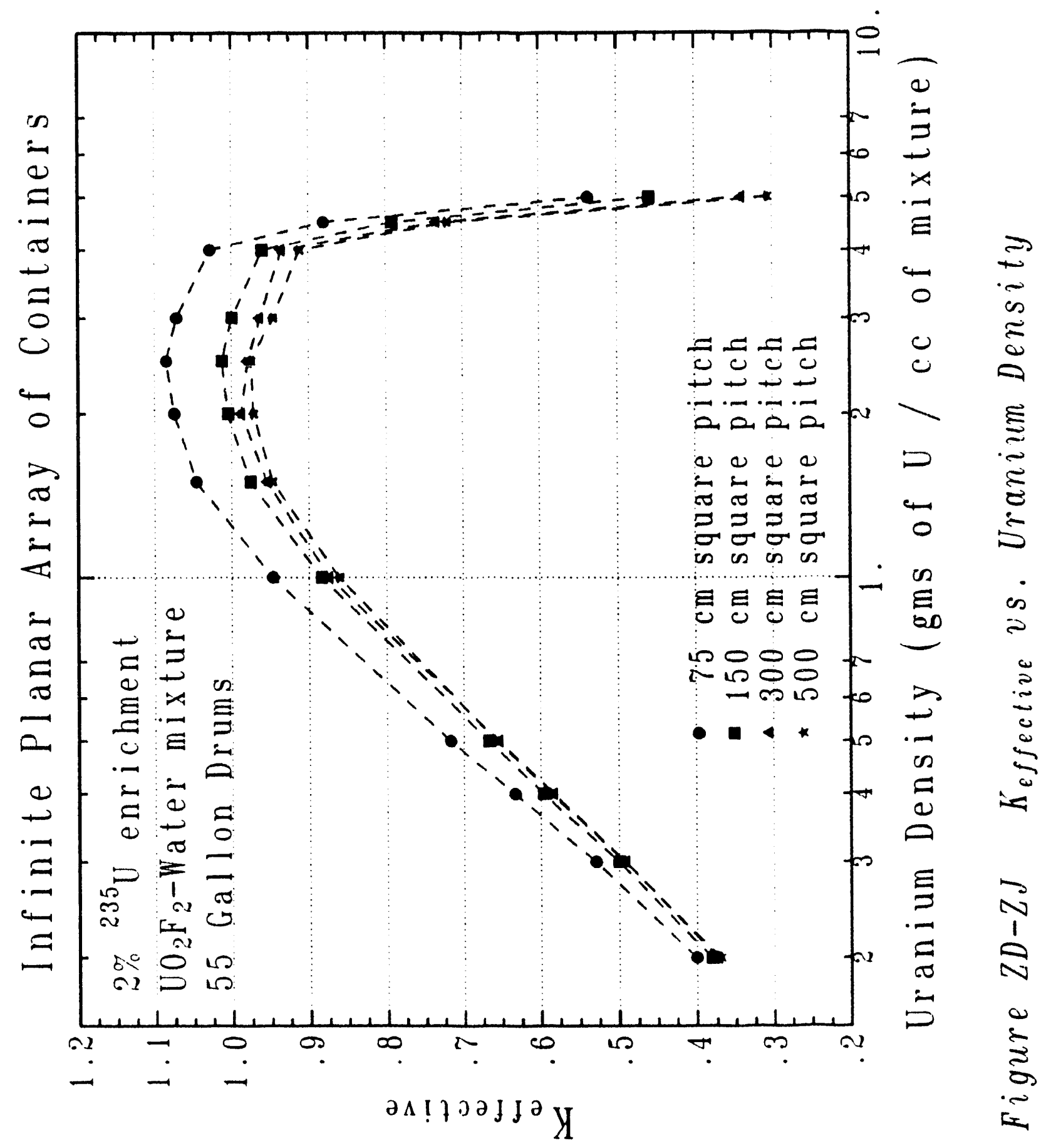


Appendix 1

\section{Material Specifications}




\section{Material Specifications for $\mathrm{UF}_{4}$ and $\mathrm{C}_{5} \mathrm{H}_{12}$ Mixture using KENO Density Multiplier Method of SCALE Section M7.5.5.4}

The KENO Density Multiplier is defined as the Actual density/Theoretical density. If we assume in this case that the volume of the mixture equals the sum of the $\mathrm{UF}_{4}$ volume and the $\mathrm{C}_{5} \mathrm{H}_{12}$ volume, than the density multiplier is the volume fraction.

Volume Fraction of $\mathrm{UF}_{4}+$ Volume Fraction of $\mathrm{C}_{5} \mathrm{H}_{12}=1.0$

Use an oil density of 0.87 gms of $\mathrm{C}_{5} \mathrm{H}_{12} / \mathrm{cc}$

Use KENO theoritical densities from SCALE Table M.8.2.1

\section{KENO Density Multiplier for UF 4}

let variable value of $\Phi=$ gms of $U / \operatorname{cc}$ of soln then :

Actual UF 4 density $=$ gms $\mathrm{UF}_{4} / \mathrm{cc}$ of soln $=\frac{\Phi \mathrm{gms} \text { of } U / \mathrm{cc} \text { of soln }}{\text { gms of } U / \text { gm of } U F 4}$

eq. $\{1\}$

For $5 \%$ enriched $\mathrm{UF}_{4} ; \quad$ gms of $U /$ gm of $\mathrm{UF}_{4}=\frac{237.89882}{237.89882+4 \cdot 18.99820}=0.7579$

For $2 \%$ enriched $\mathrm{UF}_{4} ; \quad$ gms of $\mathrm{U} / \mathrm{gm}$ of $\mathrm{UF}_{4}=\frac{237.99010}{237.98010+4 \cdot 18.99820}=0.7580$

KENO theoretical $\mathrm{UF}_{4}$ density $=6.7 \mathrm{gms}$ of $\mathrm{UF}_{4} / \mathrm{cc}$ of $\mathrm{UF}_{4}$

eq. $\{2\}$

KENO Density Multiplier for $\mathrm{UF}_{4}=$ eq. $\{1\} /$ eq. $\{2\}$

Since we assume that volume is conserved, the density multiplier is also the Volume Fraction (VF) representing ces of $\mathrm{UF}_{4} /$ cc of soln .

KENO Density Multiplier for C

The remaining Volume Fraction for the $\mathrm{C}_{5} \mathrm{H}_{12}=\left(1-\mathrm{VF}_{\text {of }} \mathrm{UF}_{4}\right)$

Actual C density $=$ gms of $\mathrm{C} / \mathrm{gm}$ of $\mathrm{C}_{5} \mathrm{H}_{12} \cdot \operatorname{gms} \mathrm{C}_{5} \mathrm{H}_{12} / \mathrm{cc} \bullet\left(1-\mathrm{VF}\right.$ of $\left.\mathrm{UF}_{4}\right)$ eq. $\{3\}$

For $\mathrm{C}_{5} \mathrm{H}_{12} ; \quad$ gms of $\mathrm{C} / \mathrm{gm}$ of $\mathrm{C}_{5} \mathrm{H}_{12}=\frac{8 \cdot 12.00000}{5 \cdot 12.0000+12 \cdot 1.00780}=0.832$

KENO theoretical $\mathrm{C}$ density $=2.3 \mathrm{gms}$ of $\mathrm{C} / \mathrm{cc}$ of $\mathrm{C}$

KENO Density Multiplier for $\mathrm{C}=$ eq. $\{3\} /$ eq. $\{4\}$

KENO Density Multiplier for $\mathrm{H}$

The remaining KENO Density Multiplier for the $\mathrm{C}_{5} \mathrm{H}_{12}=\left(1-\mathrm{VF}\right.$ of $\left.\mathrm{UF}_{4}\right)$

Actual $\mathrm{H}$ density $=$ gms of $\mathrm{H} / \mathrm{gm}$ of $\mathrm{C}_{5} \mathrm{H}_{12} \cdot \mathrm{gms} \mathrm{C}_{5} \mathrm{H}_{12} / \mathrm{cc} \bullet\left(1-\mathrm{VF}\right.$ of $\left.\mathrm{UF}_{4}\right)$ eq. $\{5\}$

For $\mathrm{C}_{5} \mathrm{H}_{12} ; \quad \mathrm{gms}$ of $\mathrm{H} / \mathrm{gm}$ of $\mathrm{C}_{5} \mathrm{H}_{12}=\frac{12 \cdot 1.00780}{3 \cdot 12.0000+12 \cdot 1.00780}=0.168$

KENO theoretical $\mathrm{H}$ density $=1.0 \mathrm{gm}$ of $\mathrm{H} / \mathrm{cc} \mathrm{of} \mathrm{H}$

eq. $\{6\}$

KENO Density Multiplier for $\mathrm{H}=$ eq. $\{5\} /$ eq. $\{6\}$ 
KENO Material Specifications for UF4-C5H12 Mixture

Using a density of 0.87 gms of $\mathrm{C5H} 12$ / cc

$2 \%$ enriched gms U / cc of soln

0.10

0.20

0.30

0.40

0.50

0.60

0.70

0.80

0.90

1.00

1.50

2.00

2.50

3.00

4.00

5.00

$5 \%$ enriched gms U / cc of soln

0.10

0.20

0.30

0.40

0.50

0.60

0.70

0.80

0.90

1.00

1.50

2.00

2.50

3.00

4.00

5.00
KENO Density Multiplier UF4

0.0197

0.0394

0.0591

0.0788

0.0985

0.1181

0.1378

0.1575

0.1772

0.1969

0.2954

0.3938

0.4923

0.5907

0.7876

0.9845

KENO Density Multiplier UF4

0.0197

0.0394

0.0591

0.0788

0.0985

0.1182

0.1379

0.1575

0.1772

0.1969

0.2954

0.3939

0.4923

0.5908

0.7877

0.9847
0.3085

0.3023

0.2961

0.2899

0.2837

0.2775

0.2713

0.2651

0.2589

0.2527

0.2218

0.1908

0.1598

0.1288

0.0668

0.0049
H

0.1433

0.1404

0.1375

0.1346

0.1318

0.1289

0.1260

0.1231

0.1203

0.1174

0.1030

0.0886

0.0742

0.0598

0.0310

0.0023 
UO2F2-H2O Densities using the Jordan/Turner Method 2 Percent Enriched UO2F2-H2O Mixture

Using KENO Masses

Mixed Uranium Molecular Weight=

Fluorine Molecular Weight=

Hydrogen Molecular Weight=

Oxygen Molecular Weight=

Water Molecular Weight=

KENO Theoretical Density for UO2F2=

KENO Theoretical Density for water=
U235 Mass $==235.04419$

U238 Mass $=238.05099$

For $H / U$ greater than 4.0

\begin{tabular}{ccccc} 
U density & H/U & UO2F2 Density & $\begin{array}{c}\text { KENO vf } \\
\text { for UO2F2 }\end{array}$ & H2O Density \\
\hline 0.2000 & 127.7908 & 0.259 & 0.041 & 0.967 \\
0.3000 & 83.8586 & 0.388 & 0.061 & 0.952 \\
0.4000 & 61.8925 & 0.518 & 0.081 & 0.937 \\
0.5000 & 48.7129 & 0.647 & 0.102 & 0.922 \\
0.6000 & 39.9265 & 0.776 & 0.122 & 0.906 \\
0.7000 & 33.6504 & 0.906 & 0.142 & 0.891 \\
0.8000 & 28.9434 & 1.035 & 0.163 & 0.876 \\
0.9000 & 25.2824 & 1.165 & 0.183 & 0.861 \\
1.0000 & 22.3536 & 1.294 & 0.203 & 0.846 \\
1.5000 & 13.5672 & 1.941 & 0.305 & 0.770 \\
2.0000 & 9.1740 & 2.588 & 0.406 & 0.694 \\
2.5000 & 6.5380 & 3.235 & 0.508 & 0.618 \\
3.0000 & 4.7807 & 3.882 & 0.609 & 0.543
\end{tabular}

For H/U less than 4.0

\begin{tabular}{llccc} 
U density & H/U & UO2F2 Density & $\begin{array}{c}\text { KENO vf } \\
\text { for UO2F2 }\end{array}$ & H2O Density \\
\hline 4.0000 & 3.0000 & 5.176 & 0.813 & 0.454 \\
4.5000 & 1.4375 & 5.823 & 0.914 & 0.245 \\
4.9600 & 0.0000 & 6.419 & 1.008 & 0.000
\end{tabular}


UO2F2-H2O Densities using the Jordan/Turner Method

5. Percent Enriched UO2F2-H2O Mixture

Using KENO Masses

Mixed Uranium Molecular Weight=

Fluorine Molecular Weight=

Hydrogen Molecular Weight=

Oxygen Molecular Weight $=$

Water Molecular Weight=

KENO Theoretical Density for UO2F2=

KENO Theoretical Density for water=
U235 Mass $==235.04419$

U238 Mass $==238.05099$

237.89882

18.9982

1.0078

15.9954

18.011

6.37

0.9982

For H/U greater than 4.0

\begin{tabular}{ccccc} 
U density & H/U & UO2F2 Density & $\begin{array}{c}\text { KENO vf } \\
\text { for UO2F2 }\end{array}$ & H2O Density \\
\hline 0.2000 & 127.7402 & 0.259 & 0.041 & 0.967 \\
0.3000 & 83.8249 & 0.388 & 0.061 & 0.952 \\
0.4000 & 61.8673 & 0.518 & 0.081 & 0.937 \\
0.5000 & 48.6927 & 0.647 & 0.102 & 0.922 \\
0.6000 & 39.9096 & 0.777 & 0.122 & 0.906 \\
0.7000 & 33.6360 & 0.906 & 0.142 & 0.891 \\
0.8000 & 28.9308 & 1.035 & 0.163 & 0.876 \\
0.9000 & 25.2712 & 1.165 & 0.183 & 0.861 \\
1.0000 & 22.3435 & 1.294 & 0.203 & 0.846 \\
1.5000 & 13.5604 & 1.941 & 0.305 & 0.770 \\
2.0000 & 9.1689 & 2.588 & 0.406 & 0.694 \\
2.5000 & 6.5340 & 3.235 & 0.508 & 0.618 \\
3.0000 & 4.7774 & 3.883 & 0.610 & 0.543
\end{tabular}

For H/U less than 4.0

\begin{tabular}{llccc} 
U density & H/U & UO2F2 Density & $\begin{array}{c}\text { KENO vf } \\
\text { for UO2F2 }\end{array}$ & H2O Density \\
\hline 4.0000 & 3.0000 & 5.177 & 0.813 & 0.454 \\
4.5000 & 1.4375 & 5.824 & 0.914 & 0.245 \\
4.9600 & 0.0000 & 6.419 & 1.008 & 0.000
\end{tabular}


Appendix 2

\section{Typical SCALE Input Files}


Scale Input for a 5 gallon container--Normal Conditions $5 \%$ enrichment $1.5 \mathrm{gm}$ of $\mathrm{U} / \mathrm{c} i$ of $\mathrm{UO}_{2} \mathrm{~F}_{2}$-Water Mixture $100 \mathrm{~cm}$ pitch

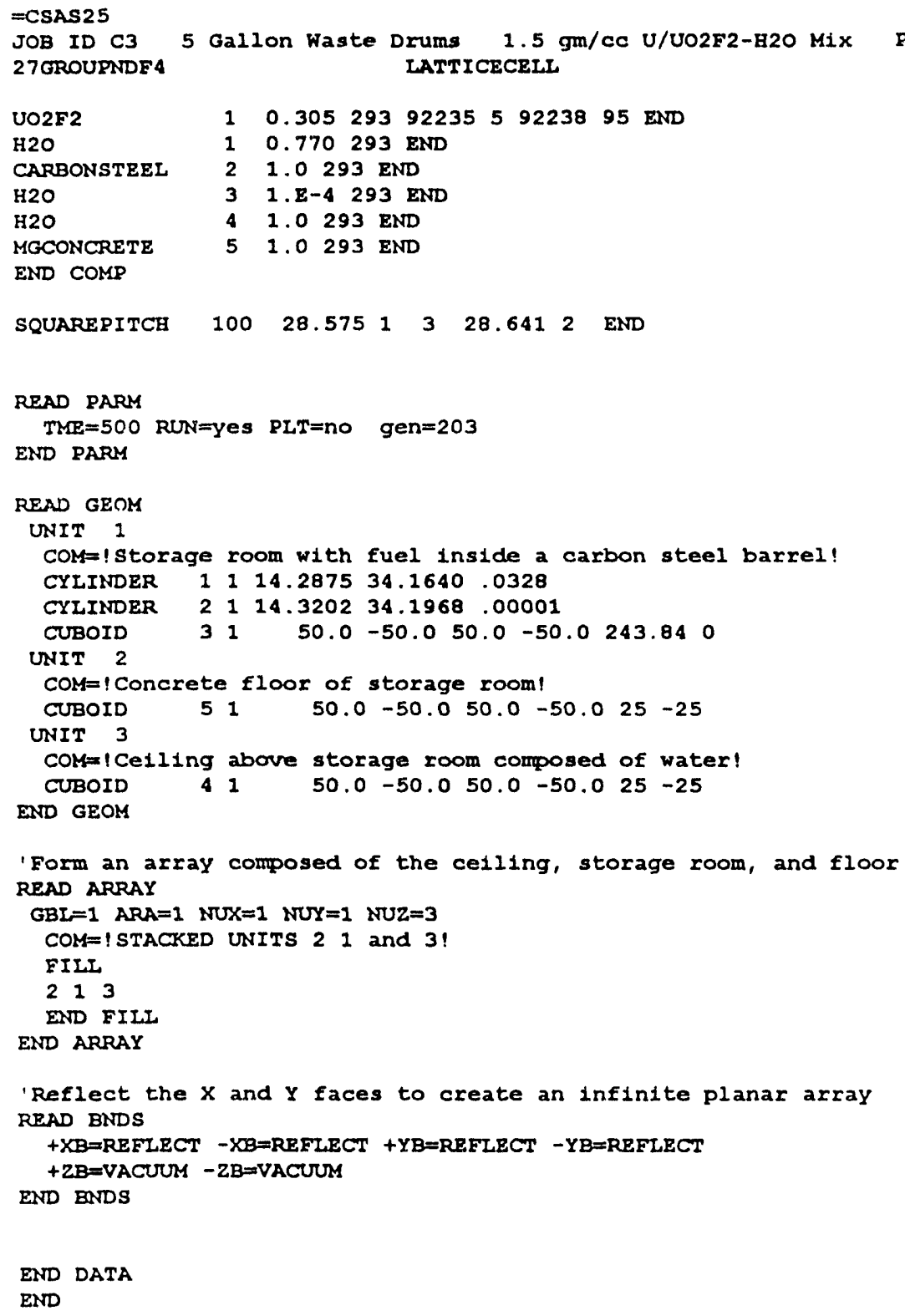




\section{Scale Input for a 5 gallon container-Normal Conditions $2 \%$ enrichment $1.5 \mathrm{gm}$ of U/cc of $\mathrm{UF}_{4}$-Oil Mixture $100 \mathrm{~cm}$ pitch}

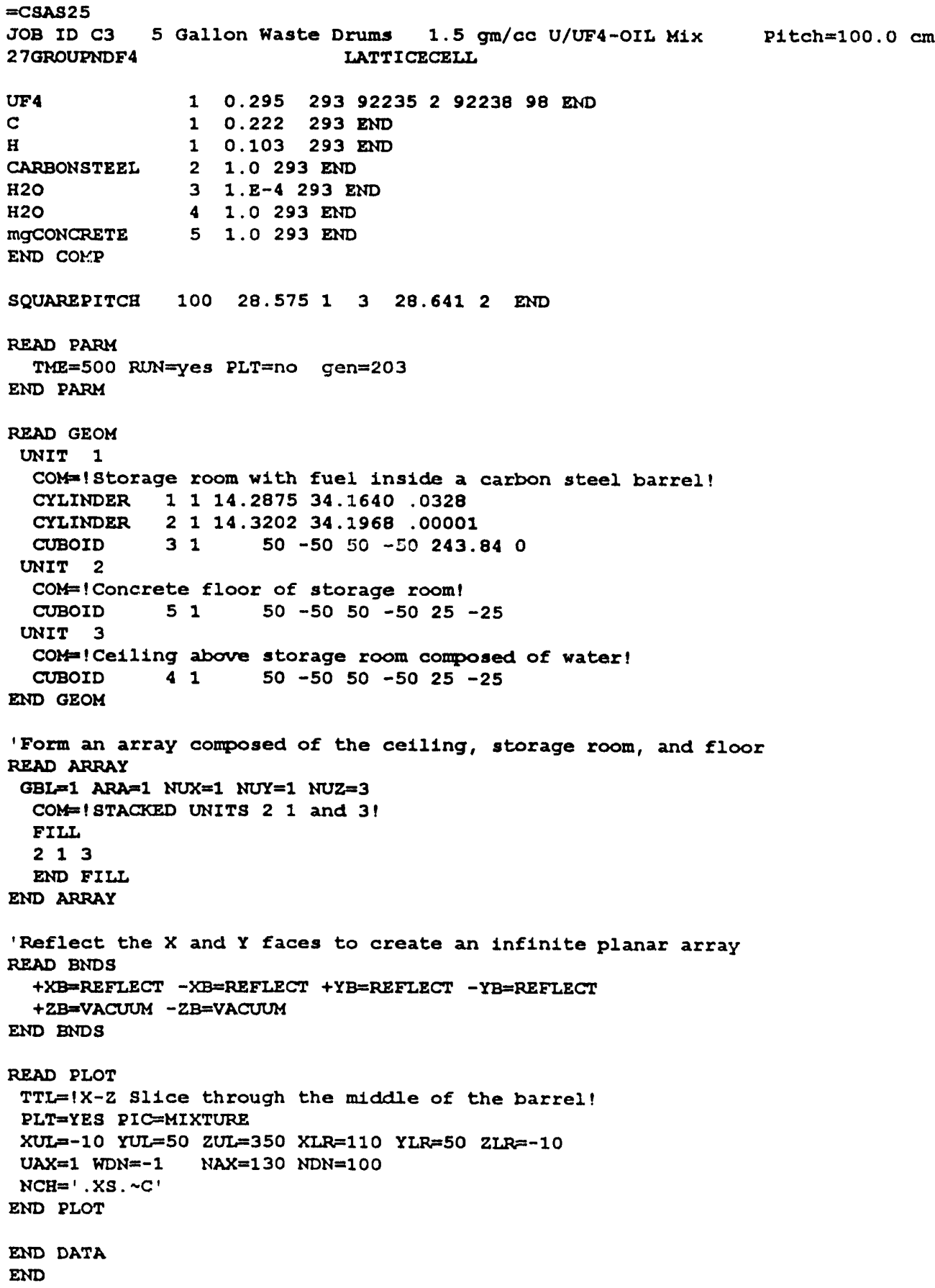


Scale Input for a 5 gallon container--Extra Interstitial Drum Accident Conditions $5 \%$ enrichment $0.4 \mathrm{gm}$ of $\mathrm{U} / \mathrm{cc}$ of $\mathrm{UO}_{2} \mathrm{~F}_{2}$-Water Mixture $100 \mathrm{~cm}$ pitch

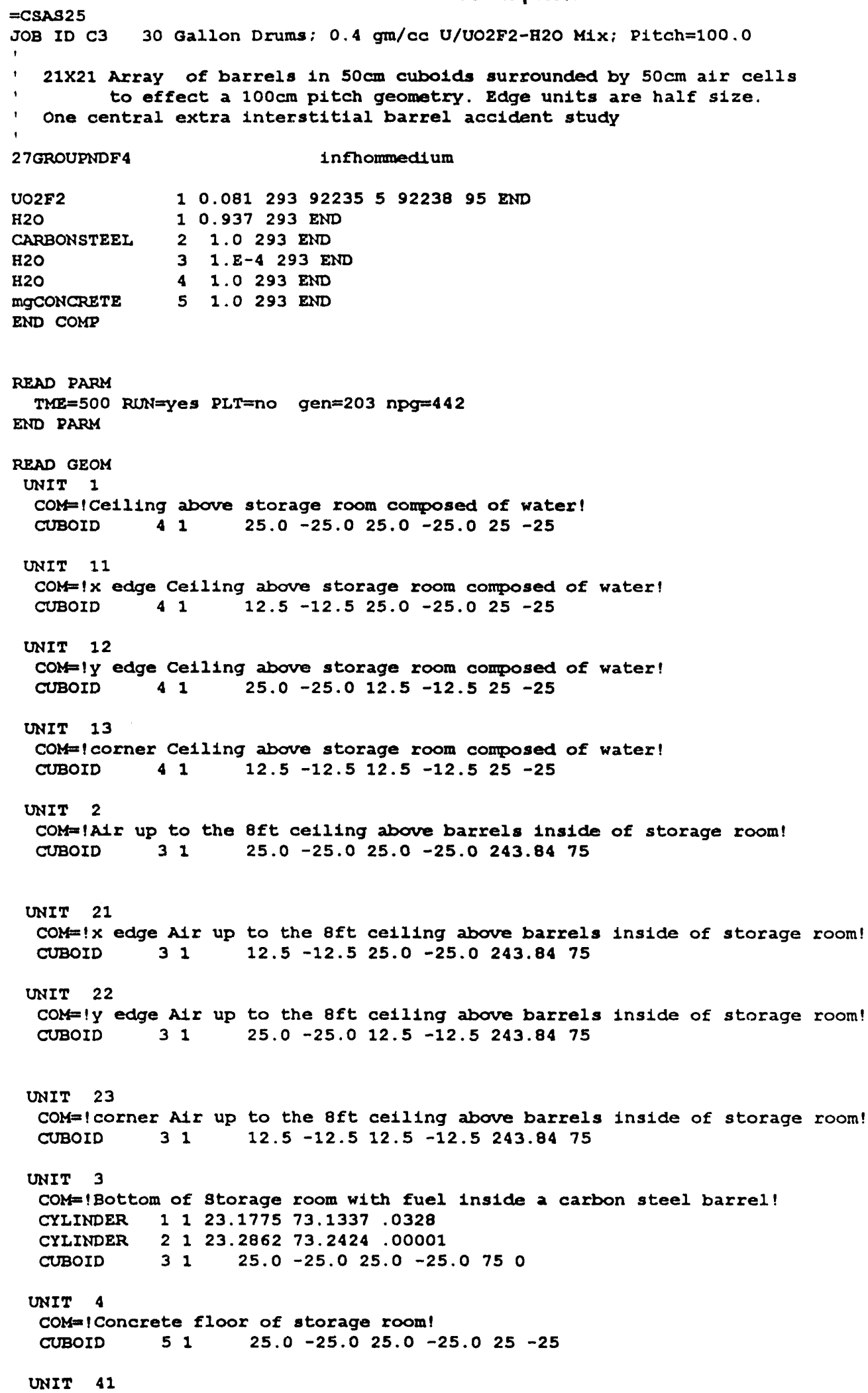




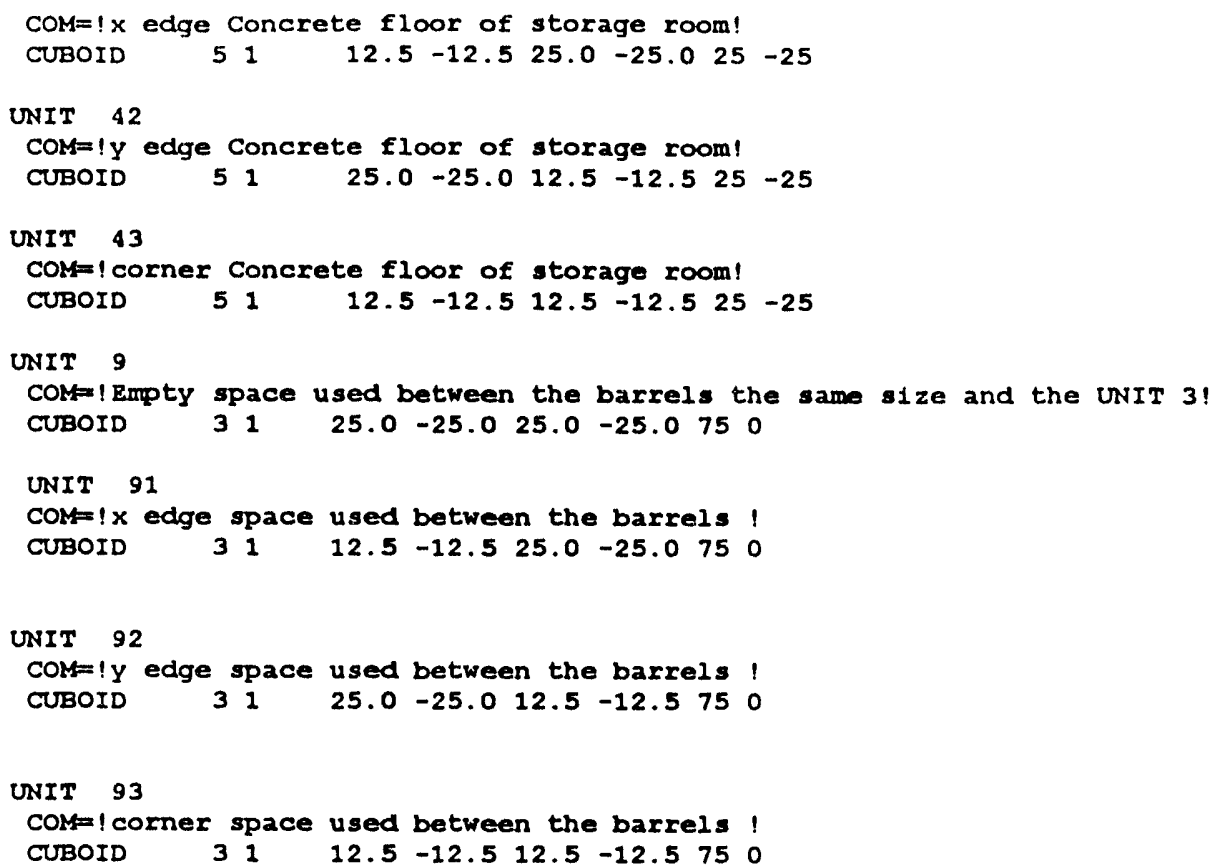

END GEOM

- Form an array composed of the celling, storage room, and floor

For the accident case substitute UNIT 3 for UNIT 9

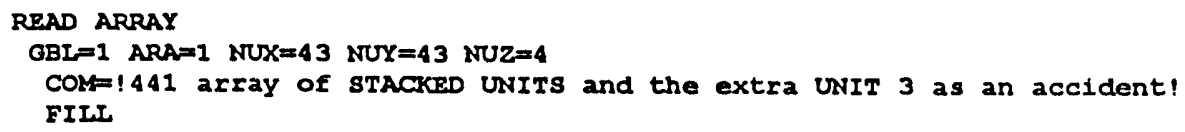


RRAD START NST $=6$

$N X S=2$ NYS $=2 \quad$ NZS $=2$

NXS $=2$ NYS $=4$ NZS $=2$

$\mathrm{NXS}=2$ NYS= $6 \mathrm{NZS}=2$

NXS $=2 \quad N Y S=8$ NZS $=2$

$\mathrm{NXS}=2 \quad \mathrm{NYS}=10 \quad \mathrm{NZS}=2$

NXS $=2$ NYS=12 NZS=2

NXS $=2$ NYS 14 NZS $=2$

$N X S=2$ NYS $=16$ NZS=2

$N X S=2 \quad N Y S=18 \quad N Z S=2$

NXS $=2$ NYS $=20 \quad$ NZS $=2$

NXS $=2$ NYS $=22 \quad N Z S=2$

NXS $=2 \quad N Y S=24 \quad N 2 S=2$

$\mathrm{NXS}=2 \quad \mathrm{NYS}=26 \quad \mathrm{NZS}=2$

$N X S=2 \quad N Y S=28 \quad$ NZS $=2$

$N X S=2$ NYS $=30 \quad$ NZS $=2$

NXS $=2$ NYS $=32 \quad$ NZS $=2$

$N X S=2$ NYS $=34 \quad N Z S=2$

NXS $=2$ NYS $=36 \quad$ NZS $=2$

$\mathrm{NXS}=2 \quad \mathrm{NYS}=38 \quad \mathrm{NZS}=2$

$N X S=2 \quad N Y S=40 \quad N Z S=2$

$\mathrm{NXS}=2$ NYS $=42 \quad \mathrm{NZS}=2$

NXS $=4 \quad$ NYS $=2 \quad$ NZS $=2$ $N X S=4$ NYS $=4$ NZS $=2$ $N X S=4 \quad N Y S=6 \quad N Z S=2$ NXS $=4$ NYS $=8$ NZS $=2$ NXS= 4 NYS=10 NZS $=2$ NXS= 4 NYS $=12$ NZS $=2$ NXS $=4 \quad$ NYS $=14 \quad$ NZS $=2$ $N X S=4 \quad N Y S=16 \quad N Z S=2$ NXS $=4 \quad N Y S=18$ NZS $=2$ $N X S=4 \quad N Y S=20$ NZS $=2$ NXS= $4 \quad N Y S=22$ NZS=2 NXS= 4 NYS=24 NZS=2 $N X S=4 N Y S=26 \quad N 2 S=2$ NXS $=4 \quad N Y S=28 \quad$ NZS $=2$ $N X 8=4 \quad N Y S=30 \quad N Z S=2$ $N X 8=4 \quad N Y S=32$ NZS $=2$

NXS $=4$ NY $8=34$ NZS=2 NXS $=4$ NYg $=36 \quad N Z S=2$ NXS $=4$ NYS $=38$ NZS $=2$ NXS $=4$ NYS $=40$ NZS $=2$ $N X S=4$ NYS $=42$ NZS $=2$

$N X S=6$ NYS $=2$ NZS $=2$ NXS- 6 NYS= 4 NZS $=2$ NXS $=6$ NYS= 6 NZS $=2$ NXS= 6 NYS $=6$ NZS $=2$ NXS $=6$ NY $=10$ NZS $=2$

$N X 8=6 \quad N Y 8=12 \quad N Z S=2$ $\mathrm{NXS}=6 \quad \mathrm{NYS}=14 \quad \mathrm{NZS}=2$ $\mathrm{NXS}=6 \quad \mathrm{NYS}=16 \quad \mathrm{NZS}=2$ $\mathrm{NXS}=6 \quad \mathrm{NYS}=18 \quad \mathrm{NZS}=2$ NXS $=6 \quad N Y S=20 \quad N Z S=2$

NXS $=6$ NXS $=22$ NZS $=2$ $\mathrm{NXS}=6 \quad \mathrm{NYS}=24 \quad \mathrm{NZS}=2$ $N X S=6 \quad N Y S=26 \quad N Z S=2$ NXS $=6 \quad$ NYS $=28 \quad$ NZS $=2$ NXS $=6$ NYS $=30 \quad \mathrm{NZS}=2$ NXS= 6 NYS $=32$ NZS $=2$ $N X 8=6 \quad N Y S=34 \quad N Z S=2$ NXS $=6 \quad$ NYS $=36 \quad$ NZS $=2$ $\mathrm{NXS}=6 \quad \mathrm{NYS}=38$ NZS $=2$ $\mathrm{NXS}=6 \quad \mathrm{NYS}=40 \quad \mathrm{NZS}=2$ NXS= 6 NYS=42 NZS=2

$N X S=8 \quad N Y 8=2 \quad N Z S=2$ NXS $=8 \quad N Y S=4 \quad N Z S=2$ $\mathrm{NX8}=8 \quad \mathrm{NY}=6 \mathrm{NZS=2}$ NX8 $=8$ NYS $=8$ NZS $=2$
$T F X=0 . T F Y=0 . T F Z=40$. LNU $=1$

$T F X=0 . T F Y=0 . T F Z=40 . \quad I N U=2$ $T F X=0 . T F Y=0 . T F Z=40 . \quad I N U=3$ $T F X=0 . T F Y=0 . T F Z=40 . \quad I N U=4$ $T F X=0$. TEY $=0 . T E Z=40$, INU $=5$ $T F X=0$. $T F Y=0$. TFZ $=40$. INU $=6$ TFX $=0$, TEY $=0$, TFZ $=40 . \quad$ INU $=7$ $T F X=0$. TFY $=0$. TFZ $=40$. LNU $=8$ $T F X=0, T F Y=0 . T F Z=40$. INUE=9 $T F X=0 . T F X=0 . T F Z=40 . \quad L N U=10$ $T F X=0$. TFY $=0$. TFZ $=40$. INU $=11$ $T F X=0 . T F Y=0 . \quad T F Z=40$. INU $=12$ $T F X=0 . T F Y=0 . T F Z=40$. INUE13 $T F X=0 . T F Y=0 . T F Z=40$. INU $=14$ $T F X=0 . T F Y=0 . T F Z=40 . \quad L N U=15$ $T F X=0$. TFY $=0$. TFZ $=40$. LNU $=16$ $T E X=0 . T F Y=0 . T E Z=40 . \quad L N U=17$ $T E X=0 . T E Y=0 . T F Z=40 . \quad$ LNUE=18 $T F X=0 . T F Y=0 . T F Z=40 . \quad L N U=19$ $T F X=0 . T F Y=0 . T F Z=40 . \quad L N U=20$ $T F X=0 . T F Y=0 . T E Z=40 . \quad I N U=21$

$T F X=0 . T F Y=0 . T F Z=40 . \quad L N U=22$ $T F X=0 . T F Y=0 . T F Z=40 . \quad I N U=23$ $T F X=0 . T F Y=0 . T F Z=40 . \quad I N U=24$ $T F X=0 . T F Y=0 . T F Z=40 . \quad I N U=25$ $T E X=0 . T F Y=0 . T F Z=40$. TNU=26 $T E X=0 . T F Y=0 . T F Z=40 . \quad I N U=27$ $T F X=0$. TFY $=0 . T F Z=40 . \quad I N U=28$ $T F X=0 . T F Y=0 . T F Z=40 . \quad I N U=29$ $T F X=0 . T F Y=0 . T F Z=40 . \quad I N U=30$ $T F X=0 . T F Y=0 . T F Z=40 . \quad I N U=31$ $T F X=0 . \quad T E Y=0 . T F Z=40 . \quad I N U=32$ $T E X=0 . T F Y=0 . T F Z=40 . \quad L N U=33$ TEX $=0 . T F Y=0 . T F Z=40 . \quad L N U=34$ $T F X=0 . T F Y=0 . T F Z=40 . \quad I N U=35$ $T F X=0 . T F Y=0 . T F Z=40$. INU $=36$ $T F X=0 . T F Y=0 . T F Z=40, \quad L N U=37$ $T F X=0$. TFY $=0 . T F Z=40 . \quad I N U=38$ $T F X=0 . T F Y=0 . T E Z=40 . \quad$ INU $=39$ $T E X=0 . T E Y=0 . T F Z=40 . \quad$ INTU 40 $T F X=0$. TFY $=0 . T F Z=40$. INU $=41$ $T F X=0 . T F Y=0 . T F Z=40 . \quad I N U=42$

$T E X=0 . T E Y=0 . T F Z=40 . \quad I N U=43$ $T F X=0 . T F Y=0 . T F Z=40 . \quad I N U=44$ $T F X=0 . T F Y=0 . T F Z=40 . \quad I N U=45$ $T F X=0 . T F Y=0 . T E Z=40 . \quad I N U=46$ $T E X=0 . T F Y=0 . T F 2=40 . \quad I N U=47$ $T F X=0 . T F Y=0 . T F Z=40 . \quad$ INU $=48$ $T F X=0 . T F Y=0, T F Z=40$. INU $=49$ $T E X=0 . T E Y=0 . T E Z=40, \quad$ LNU $=50$ $T F X=0 . T F Y=0 . T F Z=40 . \quad I N U=51$ $T F X=0 . \quad T F Y=0 . \quad T F Z=40 . \quad I N U=52$ $T E X=0 . T F Y=0 . T F Z=40 . \quad I N U=53$ $T E X=0 . T F Y=0 . T F 2=40 . \quad I N U=54$ TEX $=0$, TFY $=0 . T F Z=40 . \quad L N U=55$ $T E X=0 . T F Y=0 . T F Z=40 . \quad I N U=56$ $T E X=0 . T F Y=0 . T F Z=40 . \quad$ LNU $=57$ $T F X=0, T F Y=0 . T F Z=40 . \quad I N U=58$ $T E X=0 . T F Y=0$. TFZ $=40$. LNU $=59$ $T F X=0, T E Y=0, T E Z=40$. LNU $=60$ $T F X=0 . T F Y=0 . T E Z=40 . \quad$ LNU $=61$ $T F X=0 . T F Y=0 . T E Z=40$. INU 62 $T E X=0 . T F Y=0 . T F Z=40 . \quad L N U=63$

$T E X=0 . T F Y=0 . T F Z=40 . T N U=64$ TEX $=0 . T F Y=0 . T F Z=40 . \quad$ INU $=65$ $T F X=0$. TFY $=0$. TFZ $=40$. INU $=66$ $T E X=0 . T E Y=0 . T E Z=40 . \quad$ LNU $=67$ 
NXS $=8 \quad$ NYS $=10 \quad \mathrm{NZS}=2$ $N X S=8 \quad N Y S=12 \quad N Z S=2$ $N X S=8 \quad N Y S=14 \quad N Z S=2$ $\mathrm{NXS}=8 \quad \mathrm{NYS}=16 \quad \mathrm{NZS}=2$ $\mathrm{NXS}=\theta \quad \mathrm{NY} \mathrm{S}=18 \quad \mathrm{NZS}=2$ $N X S=B \quad N Y S=20 \quad N Z S=2$ $N X S=8 \quad N Y S=22 \quad N Z S=2$ NXS $=8$ NYS $=24$ NZS=2 $N X S=8 \quad N Y 8=26 \quad N Z S=2$ NXS= $B \quad$ NYS $=28 \quad N Z S=2$ NXS= 8 NYS=30 NZS=2 NXS $=8 \quad$ NYS=32 NZS=2 $\mathrm{NXS}=8 \quad \mathrm{NYS}=34 \quad \mathrm{NZS}=2$ NXS $=8 \quad$ NYS $=36 \quad N Z S=2$ NXS= $B \quad N Y S=38 \quad N Z S=2$ $N X S=8 \quad N Y S=40 \quad N Z S=2$ NXS= $8 \quad$ NYS $=42 \quad N Z S=2$

NXS $=10 \quad$ NYS $=2$ NZS $=2$ $N X S=10 \quad N Y S=4$ NZS=2 NXS $=10 \quad$ NYS= 6 NZS $=2$ $N X S=10 \quad N Y S=B \quad N Z S=2$ $N X S=10 \quad N Y S=10 \quad N Z S=2$ $N X S=10 \quad N Y S=12 \quad N Z S=2$ $N X S=10 \quad N Y S=14 \quad N Z S=2$ $N X S=10 \quad N Y S=16 \quad N Z S=2$ $N X S=10 \quad N Y S=18$ NZS $=2$ $N X S=10 \quad N Y 8=20 \quad N Z S=2$ $N X S=10 \quad N Y S=22 \quad N Z S=2$ NXS $=10 \quad$ NYS $=24 \quad$ NZS $=2$ NXS $=10 \quad N Y S=26 \quad N Z S=2$ $N X S=10 \quad N Y S=2 B \quad N Z S=2$ $N X S=10 \quad N Y S=30 \quad N Z S=2$ $N X S=10 \quad N Y S=32 \quad N Z S=2$ NXS $=10 \quad N Y S=34 \quad N Z S=2$ $N X S=10 \quad N Y S=36 \quad N Z S=2$ $N X S=10 \quad N Y g=38$ NZS=2 $N X S=10 \quad N Y S=40 \quad N Z S=2$ NXS $=10 \quad$ NYS $=42 \quad$ NZS $=2$

NXS $=12$ NYS= 2 NZS $=2$ $N X S=12$ NYS $=4$ NZS $=2$ $\mathrm{NXS}=12 \quad \mathrm{NYS}=6 \mathrm{NZS}=2$ $N X S=12$ NYS= $B$ NZS $=2$ $N X S=12 \quad N Y S=10 \quad N Z S=2$ $N X S=12$ NYS=12 NZS=2 $N X S=12$ NYS $=14 \quad N Z S=2$ $N X S=12$ NYS $=16 \quad N 2 S=2$ $N X S=12 \quad N Y S=18 \quad N Z S=2$ NXS $=12$ NYS $=20$ NZS $=2$ $N X S=12 \quad N Y S=22 \quad N Z S=2$ $N X S=12 \quad N Y S=24 \quad N Z S=2$ NXS $=12$ NYS $=26 \quad$ NZS=2 $N X S=12 \quad N Y S=28 \quad N Z S=2$ $N X S=12 \quad N Y S=30 \quad N Z S=2$ $N X S=12 \quad N Y S=32 \quad N Z S=2$ $N X S=12 \quad N Y S=34 \quad N Z S=2$ $N X S=12$ NYS $=36 \quad N Z S=2$ NXS $=12 \quad$ NYS $=38 \quad N Z S=2$ NXS $=12$ NYS $=40 \quad N Z S=2$ $N X S=12 \quad N Y S=42 \quad N Z S=2$

$N X S=14 \quad N Y S=2$ NZS=2 NXS=14 NYS= 4 NZS=2 $N X S=14 \quad N Y S=6 \quad N Z 8=2$ $N X S=14 \quad N Y S=8$ NZS=2 $N X S=14 \quad N Y S=10 \quad N Z S=2$ NXS=14 NYS=12 NZS=2 NX8=14 NYS=14 NZS=2 $N X S=14 \quad N Y S=16 \quad N Z S=2$ NXS=14 NYS=18 NZS=2 $N X S=14 \quad N Y S=20 \quad N Z S=2$
$T E X=0 . T F Y=0 . T F Z=40 . \quad L U U=68$ $T F X=0$. TFY $=0 . \quad T F Z=40 . \quad L N U=69$ $T F X=0$. TFY $=0$. TFZ $=40$. INNU $=70$ $T F X=0 . T F Y=0, T F Z=40 . \quad$ INU $=71$ TFX $=0$. TFY $=0$. TFZ $=40$. TNU $=72$ $T F X=0 . T F Y=0, T F Z=40 . \quad$ LNU $=73$ $T F X=0$. TEY $=0$. TFZ $=40$. LNU $=74$ $T F X=0$. TFY=0. TFZ $=40$. LNU=75 $T F X=0 . T F Y=0 . T F Z=40 . \quad L N U=76$ $T F X=0$. TFY $=0$. TFZ $=40$. LNUV=77 $T F X=0 . T E Y=0 . T F Z=40 . \quad L N U=78$ $T F X=0$. TFY $=0 . T F Z=40 . \quad L N U=79$ $T F X=0$. TFY $=0$. TFZ $=40$. LNUMBO $T F X=0, T F Y=0 . \quad T F Z=40 . \quad$ LNU $=81$ $T E X=0 . T F Y=0 . T F Z=40$. LNU=82 $T F X=0$. TFY $=0$. TFZ $T=40$. LNU $=83$ $T F X=0$. TFY $=0 . T F Z=40$. INUV=84

$T F X=0$, TFY $=0$. TFZ $=40$. INU $=85$ $T F X=0$. TEY $=0$. TFZ $=40$. LNU $=86$ $T E X=0 . T F Y=0 . T F Z=40 . \quad L N U=87$ $T F X=0$. TFY $=0 . T F Z=40 . \quad L N U=8 B$ $T F X=0$. TFY $=0$. TFZ $=40$. LNU $=89$ $T F X=0$. TFY $=0$. TFZ $=40$. LLNU $=90$ $T F X=0$. TFY $=0 . T F Z=40$. L LNU $=91$ $T F X=0$. TFY $=0$. TFZ $=40$. LNU=92 $T F X=0 . T F Y=0, T F Z=40$. LNU $=93$ $T F X=0$. TEY $=0 . T E Z=40$. LNU $=94$ $T F X=0$. TFY $=0$. TFZ $=40$. LNU $=95$ $T F X=0 . T F Y=0$. TFZ $=40$. LNU $=96$ $T F X=0$. TFY $=0$. TFZ $=40$. LNUU=97 $T F X=0$. TFY $=0 . T F Z=40 . \quad L N U=98$ $T F X=0 . T F Y=0 . T F Z=40 . \quad L N U=99$ $T F X=0$. TFY $=0 . T F Z=40 . \quad L N U=100$ $T F X=0 . T F Y=0 . T F Z=40 . \quad L N U=101$ $T F X=0$. TFY $=0$. TFZ $=40$. LNU=102 $T F X=0$. TFY $=0$. TFZ $=40 . \quad L N U=103$ $T F X=0$. $T F Y=0 . T F Z=40 . \quad I N U=104$ $T F X=0$. TFY $=0$. TFZ $=40$. LNU $=105$

$T E X=0$. TEY $=0 . T F Z=40 . \quad L N U=106$ $T F X=0$. TFY $=0$. TFZ $=40$. LNU $=107$ $T F X=0$. TFY $=0 . T F Z=40 . \quad L N U=108$ $T F X=0$. TFY=0. TFZ $=40$. LNU $=109$ $T F X=0$. TFY $=0$. TFZ $=40 . \quad L N U=110$ $T F X=0$. TEY $=0$. TFZ $=40$. LNU $=111$ $T F X=0$. TFY $=0$. TFZ $=40 . \quad L N U=112$ $T E X=0$. $T F Y=0$. TFZ=40. LNU=113 $T F X=0 . T F Y=0 . T F Z=40 . \quad L N U=114$ $T F X=0 . T F Y=0 . T F Z=40$. LNU=115 $T F X=0 . T F Y=0 . T F Z=40 . \quad L N U=116$ $T F X=0 . T F Y=0 . T F Z=40$. LNU=117 $T F X=0$. TFY $=0$. TFZ $=40$. LNU-118 $T F X=0 . T F Y=0 . T F Z=40$. LNU $=119$ $T F X=0 . T F Y=0 . T F Z=40 . \quad L N U=120$ $T F X=0 . T F Y=0 . T F Z=40 . \quad L N U=121$ $T F X=0 . T F Y=0 . T E Z=40 . \quad$ INU=122 $T F X=0$. TFY $=0 . T E Z=40$. LNU $=123$ $T E X=0 . T F Y=0 . T F Z=40$. INU $=124$ $T F X=0 . \quad T F Y=0 . \quad T F Z=40 . \quad L N U=125$ $T F X=0 . T F Y=0 . T F Z=40 . \quad I N U=126$

$T F X=0 . T F Y=0 . T F Z=40$. LNUE=127 $T F X=0 . T F Y=0 . T F Z=40 . \quad L N U=128$ $T F X=0, T F Y=0 . T F Z=40 . \quad L N U=129$ $T F X=0 . T F Y=0 . T F Z=40 . \quad L N U=130$ $T F X=0$. TFY $=0 . T F Z=40 . \quad L N U=131$ $T E X=0$. TFY $=0$. TFZ $=40$. LNU 132 $T E X=0 . T F X=0 . T F Z=40 . \quad$ LNU $=133$ $T F X=0 . T F Y=0 . T F Z=40 . \quad L N U=134$ $T F X=0$. TFY $=0$. TEZ $T=40$. LNU $T E X=0 . T F Y=0 . T F Z=40 . \quad$ LNU=136 
$N X S=14 \quad N Y S=22 \quad N Z S=2$ $N X S=14 \quad N Y S=24 \quad N Z S=2$ NXS=14 NYS=26 NZS $=2$ $N X S=14 \quad N Y S=2 B \quad N Z S=2$ $N X S=14 \quad N Y S=30 \quad N Z S=2$ $\mathrm{NXS}=14 \mathrm{NYS}=32 \mathrm{NZS}=2$ $N \times S=14 \quad N Y S=34 \quad N Z S=2$ $N X S=14$ NY $=36 \quad N Z S=2$ NXS=14 NYS $=38$ NZS=2 $N X S=14 \quad N Y S=40 \quad N Z S=2$ $N X S=14 \quad N Y 8=42 \quad N Z S=2$

$\mathrm{NXS}=16 \quad \mathrm{NYS}=2 \mathrm{NZS=2}$ $N X S=16 \quad N Y g=4 \quad N Z S=2$ $N \times S=16 \quad N Y g=6 \quad N Z S=2$ $N X 8=16 \quad N Y S=8 \quad N Z S=2$ $N X S=16 \quad N Y S=10 \quad N Z S=2$ NXS $=16 \quad N Y S=12 \quad N Z S=2$ NXS=16 NYS=14 NZS=2 $\mathrm{NXS}=16 \mathrm{NYS=16} \mathrm{NZS=2}$ $N X S=16 \quad N Y S=18 \quad N Z S=2$ NXS $=16$ NYS=20 NZS=2 $N X S=16 \quad N Y S=22 \quad N Z S=2$ NXS $=16$ NYS 24 NZS=2 $\mathrm{NXS}=16 \quad \mathrm{NYS}=26 \quad \mathrm{NZS}=2$ $\mathrm{NXS}=16 \quad \mathrm{NYS}=2.8 \mathrm{NZS}=2$ NXS $=16 \quad N Y S=30 \quad N Z S=2$ $N X S=16 \quad N Y S=32 \quad N Z S=2$ $N X 8=16 \quad N Y S=34 \quad N Z S=2$ $N X S=16 \quad N Y S=36 \quad N Z S=2$ NX8=16 NYS=38 NZS=2 $\mathrm{ITXS}=16 \quad \mathrm{NYS}=40 \quad \mathrm{NZS}=2$ $N X S=16 \quad N Y S=42 \quad N Z S=2$

$N X 8=18$ NYS $=2$ NZS $=2$ NXS $=18$ NY8 $=4$ NZS $=2$ $\mathrm{NXS}=18 \mathrm{NYS}=6 \mathrm{NZS=2}$ $N \times S=18 \quad N Y 8=8 \quad N Z S=2$ $N X S=18 \quad N Y S=10 \quad N Z S=2$ $N \times 8=18 \quad N Y 8=12 \quad N Z S=2$ $N \times S=18 \quad N Y S=14 \quad N Z S=2$ $\mathrm{NXS}=18 \quad \mathrm{NYS}=16 \quad \mathrm{NZS}=2$ $N \times S=18 \quad N Y S=18 \quad N Z S=2$ NXg=18 NY $=20 \quad N Z S=2$ $N X S=18 \quad N Y S=22 \quad N Z S=2$ NXS $=18 \quad$ NY $8=24$ NZS=2 $N \times 8=18$ NYS=26 NZS=2 NXS $=18$ NY $8=28 \quad N 2 S=2$ NXS=18 NYS=30 NZS=2 NXS $=18$ NY $=32$ NZS $=2$ NXS $=18$ NXg $=34 \quad \mathrm{NZS=}$ NXS $=18$ NYS $=36$ NZS=2 $\mathrm{NXS}=18 \quad \mathrm{NYS}=38 \quad \mathrm{NZS}=2$ $N X S=18 \quad N Y S=40 \quad N Z S=2$ $\mathrm{NXS}=18 \quad \mathrm{NYS}=42 \mathrm{NZS}=2$

$\mathrm{NXg}=20$ NYS= $2 \mathrm{NZS=2}$ $\mathrm{NXS}=20 \quad \mathrm{NYS}=4 \mathrm{NZS}=2$ $N \times S=20 \quad N Y S=6 \mathrm{NZS}=2$ $N X S=20$ NYS $=6$ NZS $=2$ $N X S=20 \quad N Y S=10 \quad N Z S=2$ $N X S=20 \quad N Y S=12 \quad N Z S=2$ $N \times 8=2.0 \quad N Y S=14$ NZS $=2$ $N \times 8=20 \quad N Y S=16 \quad N Z S=2$ $N X S=20 \quad N Y S=18 \quad N Z S=2$ $N \times S=20 \quad N X S=20$ NZS $=$ $N X S=20 \quad N Y S=22 \quad N Z S=2$ NXS $=20 \quad N Y G=24 \quad N Z S=2$ $N \times B=20 \quad N Y S=26 \quad N Z S=2$ $N X B=20 \quad$ NYS $=28 \quad N Z S=2$ $N X S=20 \quad N Y S=30$ NZS=2 $N X S=20 \quad N Y 8=32 \quad N Z S=2$
$T F X=0 . T F Y=0 . T F Z=40$. $T E X=0 . T F Y=0 . T F Z=40$. $T F X=0 . T F X=0 . T F Z=40$. $T F X=0, T E Y=0, T F Z=40$ $T F X=0 . T F Y=0 . T F Z=40$ $T F X=0$. TEY $=0, T F Z=40$ $T E X=0 . T E Y=0 . T E Z=40$ $T F X=0$, TFY $=0$. TFZ $=40$. $T F X=0, T F Y=0, T F Z=40$ $T F X=0$. TFY $=0$. TFZ $=40$ $T F X=0 . T F Y=0, T F Z=40$.

$T F X=0 . T F Y=0 . T F Z=40$. $T F X=0 . T F Y=0, T E Z=40$. $T F X=0 . T F Y=0, T F Z=40$ $T F X=0 . T F Y=0, T E Z=40$. $T F X=0 . T F Y=0, T E Z=40$. $T F X=0 . T F Y=0, T E Z=40$. $T E X=0 . T F Y=0 . T F Z=40$. $T F X=0 . T F Y=0 ， T F Z=40$ $T E X=0 . T F Y=0$. TFZ $=40$. $T E X=0, T E Y=0 \quad T F Z=40$ $T F X=0 . T F Y=0 . T F Z=40$ $T E X=0, T E Y=0, T E Z=40$. $T F X=0$. TFY $=0$. TFZ $=40$. $T E X=0 . T F Y=0 . T F Z=40$. $T E X=0 . T F Y=0$. TEZ $=40$ $T E X=0 . T F Y=0 . T E Z=40$ $T F X=0 . T F Y=0, T F Z=40$. $T F X=0, T F Y=0, T F Z=40$. TFX $=0$ ， TFY $=0, T E Z=40$ $T F X=0 . T F Y=0 . T F Z=40$ $T F X=0 . T F Y=0, T F Z=40$

$T F X=0 . T F Y=0, T F Z=40$ $T E X=0 . T F Y=0 . T F Z=40$. $T E X=0 . T F Y=0 . T E Z=40$ $T E X=0 . T F Y=0 . T F Z=40$ $T F X=0 . T F Y=0 . T F Z=40$. $T F X=0 . T E Y=0 . T E Z=40$ $T E X=0 . T F Y=0 . T E Z=40$ $T F X=0 . T F Y=0 . T F Z=40$. $T F X=0 . T F Y=0, T E Z=40$ $T E X=0 . T E Y=0 . T F Z=40$ $T F X=0 . T F Y=0 . T F Z=40$ $T E X=0 . T F Y=0 ， T F Z=40$ $T E X=0 . T F Y=0 . T F Z=40$ $T E X=0 . T E Y=0 . T E Z=40$ $T E X=0 . T F Y=0$. TEZ $=40$ $T F X=0 . T F Y=0 . T F Z=40$ $T E X=0, T F Y=0, T E Z=40$ $T E X=0 . T F Y=0, T F Z=40$ $T E X=0 \quad T F Y=0 . T F Z=40$ $T F X=0, T E Y=0, T F Z=40$ $T F X=0$. TFY $=0 . T F Z=40$

$T F X=0 . T F Y=0, T F Z=40$ $T E X=0 . T F Y=0, T E Z=40$ $T E X=0, T E Y=0, T E Z=40$ $T E X=0 . T F Y=0 . T F Z=40$ $T F X=0 . T F Y=0, T F Z=40$ $T F X=0$. TFY $=0 . T F Z=40$ $T E X=0, T F Y=0, T F Z=40$ $T E X=0, T F Y=0, T E Z=40$ $T F X=0 . T E Y=0$. TFZ $=40$ $T F X=0 \quad T E Y=0 \quad T F Z=40$ $T F X=0 . T E Y=0 . T F Z=40$ $T F X=0, T F Y=0, T F Z=40$ $T E X=0 . T F Y=0 . T F Z=40$ $T F X=0$. TFY $=0$. TFZ $=40$ $T F X=0 . T E Y=0, T E Z=40$ TEX $=0 . T F Y=0 . T F Z=40$.
LNU $=137$

$I N U=138$

LNU 139

INUE=140

INU 141

INU $=142$

INU 143

INU $=144$

INU $=145$

INU=146

INU 147

$L N U=148$

LNU $=149$

INU 150

INU 151

LNU 152

LANU 153

INU 154

INU 155

LNU=156

LNU $=157$

LNU 158

INU $=159$

INU $=160$

INU $=161$

INU $=162$

INU $=163$

INU 164

INU 165

INU 166

$L N U=167$

INU $=168$

$L N U=169$

$I N U=170$

INU 171

LNU $=172$

INU 173

LNU $=174$

LNU $=175$

INU $=176$

LNUE=177

LNU 178

INUE=179

$I N U=180$

LNU 181

LNU 182

LNU 183

LNU 184

LNU 185

INU $=186$

INUV $=187$

$\mathrm{LNU}=188$

$L N U=189$

LNU=190

LNU 191

LNU=192

LNU 193

INUE=194

INU $=195$

INU 196

LNU $=197$

LNU $=198$

INUE 199

INU 200

$L N U=201$

INU $=202$

LNU $=203$

INU 204

INU 205 
$N X S=20 \quad$ IYS $=34 \quad N Z S=2$ $N X S=20 \quad N Y S=36 \quad N Z S=2$ $N X S=20 \quad$ NYS $=38 \quad N Z S=2$ $N X S=20 \quad N Y 8=40 \quad N 2 S=2$ $N X S=20 \quad N Y S=42 \quad N Z S=2$

NXS=22 NYS= 2 NZS=2 $N X g=22 \quad N Y S=4 N Z S=2$ $N X S=22$ NYS -6 NZS=2 $N X 8=22 \quad N Y 8=8$ NZS=2 $N \times 8=22 \quad N Y 8=10 \quad N Z g=2$ NXS 22 NYS $=12$ NZS $=2$ $N X S=22 \quad N Y S=14 \quad N Z S=2$ $N X S=22 \quad N Y S=16 \quad N Z S=2$ $N X S=22 \quad N Y S=18 \quad N Z S=2$ $N X S=22 \quad N Y B=20 \quad N Z S=2$ NX8 22 NYS $=22$ NZS=2 $N X S=22 \quad N Y S=24 \quad N Z S=2$ $N X S=22 \quad N Y S=26 \quad N Z S=2$ $N X S=22 \quad N Y 8=28 \quad N Z S=2$ $N X S=22 \quad N Y S=30 \quad N Z S=2$ $N X S=22 \quad N Y S=32 \quad N Z S=2$ $N X S=22 \quad N Y S=34 \quad N Z S=2$ $N X S=22 \quad N Y S=36 \quad N Z S=2$ $N X S=22 \quad N Y S=38 \quad N Z S=2$ $N X S=22 \quad N Y S=40 \quad N Z S=2$ $N X S=22 \quad N X S=42 \quad N Z S=2$

NXS $=24$ NYS= 2 NZS=2 $N X S=24 \quad$ NYS $=4 \quad N Z S=2$ NXS $=24$ NYS $=6$ NZS $=2$ $N X S=24 \quad N Y S=\theta \quad N Z S=2$ NXS $=24 \quad$ NYS $=10 \quad$ NZS $=2$ $N X S=24 \quad N Y S=12 \quad N Z S=2$ $N X S=24 \quad N Y S=14 \quad N Z S=2$ $N X g=24 \quad N Y 8=16 \quad N Z S=2$ NXS $=24 \quad N X 8=18 \quad$ NZS $=2$ NXS $=24 \quad N Y 8=20 \quad N Z S=2$ $N X 8=24 \quad N Y g=22 \quad N Z S=2$ NXS $=24$ NYS=24 NZS=2 NX8 $=24 \quad N Y 8=26 \quad N Z 8=2$ NXS $=24 \quad N Y S=28 \quad N Z 8=2$ NXS $=24$ NYS $=30$ NZS $=2$ $N X S=24 \quad N Y S=32 \quad N Z 8=2$ NXS $=24 \quad$ NYS $=34 \quad$ NZS=2 $N X S=24 \quad N Y 8=36 \quad N 28=2$ NXS $=24$ NYS $=38$ NZS $=2$ $N X S=24 \quad N Y 8=40 \quad N Z 8=2$ NXS $=24$ NYS $=42 \quad N Z S=2$

$N X S=26 \quad N Y S=2 \quad N Z S=2$ $N X S=26 \quad N Y S=4 \quad N Z S=2$ $N X S=26 \quad N Y S=6 \quad N Z S=2$ NXS $=26 \quad$ NYS $=8$ NZS $=2$ NXS $=26 \quad N Y S=10 \quad$ NZS $=2$ $N X S=26 \quad N Y S=12 \quad N Z S=2$ NXS $=26 \quad N Y S=14 \quad N Z S=2$ NXS $=26$ NYS $=16 \quad$ NZS=2 $N X S=26 \quad N Y S=18 \quad N Z S=2$ $N X 8=26 \quad N Y 3=20 \quad N Z S=2$ NXS $=26 \quad N Y S=22 \quad N Z S=2$ NXS $=26 \quad$ NYS $=24 \quad$ NZS $=2$ NXS $=26 \quad$ NYS $=26 \quad$ NZS $=2$ $N X S=26 \quad N Y S=28 \quad N Z S=2$ $N X g=26 \quad N Y S=30 \quad N Z S=2$ $N X S=26 \quad N Y S=32 \quad N Z S=2$ $N X 8=26 \quad N Y 8=34 \quad N Z 8=2$ $N X S=26 \quad N Y S=36 \quad N Z 8=2$ $N X 8=26 \quad N Y 8=38 \quad \mathrm{NZS}=2$ $N X 8=26 \quad N Y S=40 \quad$ NZS $=2$ NXS $=26 \quad N Y S=42 \quad N Z S=2$
$T F X=0 . T F Y=0, T F Z=40$. $T F X=0$. TFY $=0$. TFZ=40. $T E X=0, T F Y=0 . T E Z=40$. $T E X=0 . T F Y=0 . T F Z=40$. $T F X=0$. TFY $=0$. TFZ $=40$.

$T E X=0$. TFY $=0 . T F Z=40$. $T E X=0 . T F Y=0 . T F Z=40$. $T F X=0$. TFY $=0, T E Z=40$. $T F X=0$. TFY $=0$. TFZ $=40$. $T F X=0 . T E Y=0 . T E Z=40$. $T F X=0 . T F Y=0 . T F Z=40$. $T F X=0 . T F Y=0 . T F Z=40$. $T F X=0 . T F Y=0 . T F Z=40$. $T F X=0$. TFY $=0$. TFZ $=40$. $T F X=0 . T F Y=0 . T F Z=40$ $T F X=0$. TFY $=0$. TFZ $=40$ $T F X=0$. TFX $=0$. TFZ $=40$. $T E X=0$. TFY $=0$. TFZ $=40$ $T F X=0, T F Y=0, T E Z=40$ $T F X=0$. TFY $=0$. TFZ $=40$. $T E X=0$. TFY $=0$. TFZ $=40$. $T F X=0 . T F Y=0 . T F Z=40$. $T F X=0$. TFY $=0$. TFZ $=40$ $T E X=0 . T F Y=0 . T E Z=40$ $T E X=0$. TFY=0. $T E Z=40$ $T E X=0, T E Y=0 . T E Z=40$.

$T F X=0$. TFY $=0$. TFZ $=40$. $T E X=0$. TFY $=0$. TFZ $=40$. $T E X=0$. TFY $=0$. TFZ=40. $T E X=0$. TFY $=0$. TEZ $T=40$. $T F X=0 . T F Y=0 . T F Z=40$. $T E X=0$. TEY $=0$. TFZ $=40$. $T E X=0 . T F Y=0 . T E Z=40$ $T F X=0 . T F Y=0$. TEZ $=40$. $T F X=0$. TFY $=0$. TFZ $=40$. $T F X=0 . T E Y=0 . T P Z=40$. $T F X=0 . T E Y=0 . T F Z=40$. $T E X=0$. TEY $=0 . T F Z=40$. $T E X=0 . T E Y=0 . T E Z=40$. $T E X=0$. TFY $=0$. TEZ $=40$. $T F X=0$. TFY $=0$. TPZ=40. $T E X=0$. TFY $=0$. TFZ $=40$. $T F X=0 . T F Y=0 . T F Z=40$. $T E X=0 . T F Y=0 . T F Z=40$. $T E X=0 . T F Y=0 . T F Z=40$. $T E X=0$. TFY $=0$. TEZ $=40$. $T F X=0$. TFY $=0$. TFZ $=40$.

$T F X=0 . T F Y=0 . T F Z=40$. $T F X=0 . T F Y=0 . T F Z=40$. $T F X=0 . T E Y=0$. TFZ $=40$. $T F X=0 . T F Y=0 . T F Z=40$.

$T E X=0 . T F Y=0$. TFZ $=40$. $T F X=0 . T F Y=0 . T F Z=40$. $T F X=0 . T F Y=0 . T F Z=40$. $T F X=0 . T F Y=0 . T F Z=40$. $T E X=0 . T E Y=0 . T E Z=40$ $T E X=0 . T E Y=0 . T F Z=40$ $T F X=0$. $T F Y=0 . T F Z=40$. $T E X=0 . T E Y=0 . T E Z=40$ $T E X=0$. TFY $=0 . \quad T E Z=40$. $T F X=0$. TFY $=0 . T E Z=40$. $T F X=0$. TFY $=0$. TFZ $=40$. $T E X=0$. TFY $=0$. TFZ $=40$. $T F X=0$. TFY $=0, T F Z=40$ $T F X=0 . T F Y=0 . T F Z=40$. $T F X=0 . T F Y=0 . T F Z=40$. $T F X=0 . T F Y=0 . \quad T F Z=40$. $T F X=0 . T F Y=0 . T F Z=40$.
LNU 206 LNU $=207$ $L N U=208$ LNU 209 LNU $=210$

LNU $=211$ LNU 212

$L N U=213$

LNU 214

LNU 215

LNU 216

LNU 217

LNU $=218$

LNU $=219$

LNU 220

LNU $=221$

LNU 222

$L N U=223$

LNU $=224$

INU $=225$

LNU $=226$

$L N U=227$

LNU $=228$

$I N U=229$

$I N U=230$

INU $=231$

$L . N U=232$

$L N U=233$

$L N U=234$

INU $=235$

LNU $=236$

$I N U=237$

LNU $=238$

LNU $=239$

LNU 240

INU $=241$

$I N U=242$

LNU 243

LNU $=244$

INU 245

LNU 246

INU 247

$L N U=248$

INU 249

$I N U=250$

INU 251

$L N U=252$

$L N U=253$

LNU $=254$

LNU $=255$

LNUE=256

L $U=257$

$L N U=258$

LNU $=259$

INU $=260$

LNU $=261$

INU $=262$

$L N U=263$

LNU 264

INU 265

LNU $=266$

INU $=267$

$L N U=268$

INU $=269$

INU $=270$

LNU $=271$

LNU 272

$L N U=273$ 
$N X g=28 \quad N Y S=2 N Z=2$ $N X g=28 \quad N Y g=4 N Z g=2$ $N X S=28 \quad N Y S=6 \quad N Z S=2$ NXg=28 NYS= $8 \mathrm{NZS=2}$ NXS $=28 \quad N Y 8=10 \quad N Z S=2$ $N X 8=28$ NYg 12 NZS=2 NX8 $=28 \quad$ NY $8=14$ NZ8=2 $N X g=28 \quad N Y g=16 \quad N 28=2$ NXg $=28$ NYS 18 NZS=2 NXg $=28 \quad N Y g=20$ NZ8 $=2$ $N \times S=28 \quad N Y g=22 \quad N 2 S=2$ $N X 8=28 \quad N Y g=24 \quad N Z S=2$ NXS $28 \quad N Y S=26 \quad N 2 S=2$ $N X g=28 \quad N Y g=28 \quad N Z S=2$ $N X 8=28 \quad N Y S=30 \quad N Z S=2$ NXS $=28 \quad N Y S=32$ NZS=2 $N X S=28 \quad N Y S=34 \quad N Z g=2$ NXS $=28 \quad N Y S=36 \quad N Z S=2$ NXS $=28$ NYS $=38$ NZS=2 $N \times g=28 \quad N Y S=40 \quad N Z S=2$ NXS $=28$ NYS $=42 N Z S=2$

NXS-30 NYS 2 NZB=2 $N X S=30 \quad N Y S=4 N Z S=2$ NXS=30 NYS= 6 NZS $=2$ NXS $=30$ NYS $=8$ NZS $=2$ NXS $=30 \quad N Y S=10 \quad N Z S=2$ NXS=30 NYS=12 NZS=2 $N X S=30 \quad N Y 8=14 \quad N 2 S=2$ $N X g=30 \quad N Y S=16 \quad N 2 S=2$ $N X 8=30 \quad N Y 8=18 \quad N Z g=2$ $N X S=30 \quad N Y S=20 \quad N Z S=2$ $N X 8=30 \quad N Y S=22 \quad N Z S=2$ $N X S=30 \quad N Y S=24 \quad N Z S=2$ NX8 $=30 \quad N Y S=26 \quad N Z S=2$ $N \times g=30 \quad N Y 8=28 \quad N Z S=2$ $N X s=30 \quad N Y s=30 \quad N Z S=2$ NXg $=30 \quad$ NY $8=32 \quad$ NZS $=2$ NXg=30 NYg=34 NZS=2 NXS $=30 \quad N Y 8=36$ NZS=2 NX8 $=30 \quad$ NY $8=38$ NZS=2 $N X S=30 \quad N Y 8=40 \quad N 2 s=2$ $N X g=30 \quad N Y S=42 \quad N Z s=2$

$\mathrm{NXS}=32 \mathrm{NYS}=2 \mathrm{NZS}=2$ $N \times S=32$ NYS $=4 N 2 S=2$ $\mathrm{NXS}=32 \mathrm{NYS}=6 \mathrm{NZS}=2$ NXS $=32$ NYS= 8 NZS $=2$ $N X S=32 \quad N Y S=10 \quad N Z S=2$ NXS $=32$ NYS $=12$ NZS $=2$ $N X S=32 \quad N Y S=14 \quad N Z S=2$ $N X S=32 \quad N Y S=16 \quad N Z S=2$ $\mathrm{NXS}=32 \mathrm{NYg}=18 \mathrm{NZS}=2$ $N X 8=32 \quad N Y S=20 \quad N Z S=2$ $\mathrm{NXS}=32 \mathrm{NYS}=22 \mathrm{NZS}=2$ NXS $=32 \quad N Y S=24 \quad N Z S=2$ $N X g=32 \quad N Y S=26 \quad N Z S=2$ $\mathrm{NXS}=32 \quad \mathrm{NY} 8=28 \quad \mathrm{NZS}=2$ $N \times 8=32 \quad N Y 8=30 \quad N 28=2$ $N \times 8=32 N Y g=32$ NZS $=2$ $N \times S=32 N \times g=34 \quad N 2 S=2$ NXS $=32 \quad N Y 8=36 \quad$ NZS $=2$ NXS $=32 \quad N Y S=38 \quad N 2 S=2$ $N \times 8=32 \quad N Y S=40 \quad N Z S=2$ $N \times g=32 N Y g=42 \quad N 2 g=2$

NXS $=34$ NYS $=2$ NZS $=2$ NXS $=34$ NYS= 4 NZS=2 NX8=34 NY8 6 NZSm2 NXS $=34$ NY $=8$ NZS $=2$ NXS=34 NYS=10 NZS=2 $N X S=34 \quad N Y 8=12 \quad N Z S=2$
$T E X=0 . T F Y=0 . T F Z=40$. $T F X=0, T F Y=0, T E Z=40$. $T F X=0 . T F Y=0, T F Z=40$. $T E X=0 . T F Y=0 . T E Z=40$. $T F X=0$. TFY $=0$. TFZ $=40$ $T F X=0 . T E Y=0$. TEZ $=40$. $T E X=0 . T E Y=0, T F Z=40$. $T E X=0 . T F Y=0 . T E 2=40$ $T E X=0 . T E Y=0 . T F Z=40$. $T E X=0, T E Y=0, T E Z=40$. $T E X=0 . T E Y=0 . T F Z=40$. $T E X=0$. TFY $=0$. TFZ $=40$. $T E X=0, T E Y=0 . T F Z=40$ $T F X=0$. TFY $=0$. TFZ $=40$. $T F X=0$. TEY $=0$. TFZ $=40$ $T F X=0, T F Y=0 . T F Z=40$ $T E X=0 . T E Y=0 . T F Z=40$. $T F X=0 . T E Y=0, T F Z=40$ $T E X=0 . T E Y=0 . T F Z=40$ $T E X=0 . T E Y=0, T E Z=40$ TEX $=0 . T E Y=0, T F Z=40$

$T E X=0 . T E Y=0 . T F Z=40$. $T F X=0, T F Y=0, T E Z=40$ $T F X=0 . T F Y=0 . T F Z=40$ $T E X=0, T E Y=0, T F Z=40$ $T E X=0 . T E Y=0 . T F Z=40$. $T E X=0 ， T E Y=0 ， T F Z=40$ $T F X=0 . T F Y=0 . T E Z=40$ $T E X=0 . T F Y=0 . T E Z=40$. $T E X=0 . T E Y=0, T E Z=40$. $T E X=0 . T E Y=0 . T E Z=40$ $T F X=0 ， T F Y=0 ， T E Z=40$ TFX $=0$. TFY $=0$. TFZ $=40$ $T F X=0 . T F Y=0 . T F Z=40$. $T F X=0 . T E Y=0, T E Z=40$. $T F X=0 . T E Y=0 . T F Z=40$. $T E X=0 . T F Y=0 . T E Z=40$ $T F X=0 . T F Y=0 . T F Z=40$ $T F X=0 . T E Y=0 . T F Z=40$ $T E X=0 . T F Y=0 . T F Z=40$. $T F X=0 . T F Y=0 . T F Z=40$. $T E X=0 . T F Y=0 . T F Z=40$

$T F X=0 . T F Y=0 . T F Z=40$. $T F X=0, T F Y=0 . T E Z=40$. TFX $=0$. TFY $=0$. TFZ $=40$. $T F X=0$, TFY $=0$, TFZ $=40$ $T E X=0 . T F Y=0, T E Z=40$ $T F X=0 . T F Y=0 . T F Z=40$. $T E X=0$. TEY $=0$, TFZ $=40$ $T E X=0, T E Y=0 . T F Z=40$ $T F X=0 . T F Y=0 . T F Z=40$. TFX $=0$, TEY $=0$. TEZ $=40$. $T E X=0 . T F Y=0 . T F Z=40$. $T E X=0 . T F Y=0, T F Z=40$ $T F X=0 . T F Y=0 . T F Z=40$ $T E X=0$. TEY $=0$. TEZ $=40$. $T F X=0 . T F Y=0 . T E Z=40$ $T F X=0 . T F Y=0 . T E Z=40$. $T F X=0, T E Y=0, T F Z=40$ $T F X=0 . T F Y=0 . T F Z=40$ $T F X=0, T F Y=0 . T F Z=40$ $T E X=0, T E Y=0, T F Z=40$ $T F X=0$. TFY $=0$. TFZ $=40$.

$T E X=0, T E Y=0 . T F Z=40$. $T F X=0 . T F Y=0 . T F Z=40$. $T F X=0, T E Y=0, T E Z=40$ $T F X=0 . T E X=0 . T E Z=40$. $T E X=0, T E Y=0 . T F Z=40$ $T F X=0$. TFY $=0$. TFZ $=40$.
LNU 274

LNO=275

INU 276

INU 277

INUM 278

LNU 279

INUW 280

INU 281

LNU 282

INU 283

INUE=284

LNU $=285$

LNU 286

INU 287

$I N U=288$

LNU 289

INU $=290$

INU $=291$

LNU 292

INU 293

LNU $=294$

LNU 295

INU=296

INU $=297$

INU $=298$

INU=299

LNU $=300$

INUm 301

INU 302

$L N U=303$

$L N U=304$

INU $=305$

INU $=306$

INU 307

LNU $=308$

INU 309

INU $=310$

LNU 311

INU 312

LNUD313

INUG=314

LNU $=315$

INU $=316$

LNOU=317

INU $=318$

INU=319

$I N U=320$

LNU $=321$

LNU=322

LNU $=323$

LNU 324

INU 325

INU $=326$

$I N U=327$

$L N U=328$

INU $=329$

$I N U=330$

INU $=331$

INU 332

LNO $=333$

LNU $=334$

LNU $=335$

LNU:336

LNO $=337$

LNU $=338$

LNU 339

INU $=340$

INTU-341

INU 342 
$\mathrm{NXS}=34 \quad \mathrm{NYS}=14 \mathrm{NZS=2}$ NXS $=34$ NYS $=16 \quad N 2 S=2$ $N X S=34 \quad N Y S=18 \quad N Z S=2$ $N X S=34 \quad N Y B=20 \quad N Z S=2$ NXS $=34 \quad N Y S=22 \quad N Z 8=2$ NXg $=34$ NYg= $24 \quad N Z S=2$ NXS=34 NY $8=26 \quad$ NZS=2 $N \times 8=34 \quad N Y 8=28 \quad N Z S=2$ $\mathrm{NX8}=34 \quad \mathrm{NYg}=30 \quad \mathrm{NZS}=2$ $N \times g=34 \quad N Y g=32 \quad N Z S=2$ NXS=34 NYg=34 NZS=2 $N X 8=34 \quad N Y S=36 \quad N Z S=2$ NXS $=34 \quad N Y S=38$ NZS $=2$ $\mathrm{NXS}=34 \quad \mathrm{~N} \times \mathrm{S}=40 \mathrm{NZS}=2$ $N X S=34 \quad N Y S=42 \quad N Z S=2$

INXS $=36$ NYS= 2 NZg=2 NXS=36 NYS= 4 NZS=2 NXS $=36$ NYS= 6 NZS $=2$ NXS=36 NYS= $\mathrm{NZS}=2$ $N X S=36 \quad N Y S=10 \quad N Z S=2$ NXS $=36 \quad N Y 8=12 \quad N 2 S=2$ $N X g=36 \quad N Y S=14$ NZS=2 $N X S=36 \quad N Y S=16 \quad N Z S=2$ NXS $=36 \quad N Y S=18 \quad N 2 S=2$ $N X S=36 \quad N Y S=20 \quad N Z S=2$ NXS=36 NYS=22 NZS=2 $N \times g=36 \quad N Y g=24 \quad N z S=2$ $N \times 8=36 \quad N Y S=26 \quad N Z S=2$ NXS=36 NX8=28 NZS=2 $\mathrm{NX8}=36 \quad \mathrm{NXg}=30 \mathrm{NZS}=2$ NXS $=36 \quad N Y S=32$ NZS $=2$ $\mathrm{NXS}=36 \quad \mathrm{NYS}=34 \mathrm{NZS}=2$ $N \times S=36 \quad N Y g=36 \quad N Z S=2$ NX8 $=36 \quad \mathrm{NYS}=38 \quad \mathrm{NZS}=2$ NXS $=36 \quad \mathrm{NYS}=40 \quad \mathrm{NZS}=2$ NXS $=36 \quad N Y S=42 \quad N Z S=2$

NXS $=38$ NY $=2$ NZ8=2 NX8 $=38$ NY8 $=4$ NZg=2 NXS $=38$ NYS $=6$ NZS $=2$ $N \times S=38 \quad N Y S=8 \quad N Z S=2$ $\mathrm{NXS}=38 \quad \mathrm{NY} 8=10 \quad \mathrm{NZS}=2$ NXS $=38 \quad N Y S=12 \quad N Z S=2$ $N X 8=38$ NY $8=14$ NZS=2 NXS $=38$ NXs $=16$ NZS=2 NXS $=38$ NY $=18 \quad$ NZS $=2$ NXS $=38$ NY $8=20$ NZS $=2$ NXS $=38$ NY $8=22 \quad$ NZS $=2$ NXS-38 NY8 $=24$ NZg $=2$ NX8 $=38 \quad N Y 8=26$ NZS $=2$ NX $8=38$ NY $8=28$ NZS $=2$ NXS $=38$ NY8 $=30$ NZS=2 NXS $=38$ NYS $=32$ NZS $=2$ NXS $=38$ NY $8=34 \quad N Z S=2$ NXS=38 NXS=36 NZS=2 NXS $=38 \quad N Y S=38 \quad N Z S=2$ NXS $=38 \quad N Y 8=40 \quad N Z S=2$ $N X S=38 \quad N Y S=42 \quad N Z g=2$

$N \times 8=40 \quad N Y B=2 N Z S=2$ $N \times 8=40$ NY $=4$ NZS $=2$ $N \times S=40$ NYS= 6 NZS $=2$ $N X S=40 \quad N Y g=8$ NZS $=2$ $N X 8=40 \quad N Y 8=10 \quad N Z S=2$ $N X S=40 \quad N Y S=12 \quad \mathrm{NZS}=2$ $N X S=40 \quad N Y S=14 \quad N Z S=2$ $N \times S=40 \quad N \times S=16 \quad N 2 S=2$ $N X S=40 \quad N Y S=18 \quad N Z g=2$ $N \times 8=40 \quad N Y S=20 \quad N Z S=2$ $N \times 8=40 \quad N Y g=22$ NZS=2 $N X s-40$ NY $8=24 \quad N Z S=2$
$T F X=0 . T F Y=0 . T F Z=40$. $T F X=0 . T F Y=0 . T F Z=40$. $T F X=0 . T F Y=0 . T F Z=40$. $T F X=0, T E Y=0 . T F Z=40$. $T F X=0, T E Y=0$. TEZ $=40$. $T E X=0 . T E Y=0 . T E Z=40$. $T E X=0, T E Y=0 . T F Z=40$. $T E X=0$. TFY $=0$. TFZ $=40$ $T E X=0 . T F Y=0 . T F Z=40$. $T F X=0 . T F Y=0 . T F Z=40$. $T F X=0 . T F Y=0 . T F Z=40$. $T E X=0 . T E Y=0 . T E Z=40$ $T F X=0, T E Y=0 . T E Z=40$ $T E X=0$. TEY $=0$. TFZ $=40$ $T E X=0 . T F Y=0 . T E Z=40$.

$T E X=0 . T F Y=0, T E Z=40$ $T F X=0 . T E Y=0, T E Z=40$ $T F X=0 . T E Y=0 . T E Z=40$. $T E X=0, T E Y=0, T E Z=40$ $T F X=0 . T E Y=0$. TEZ $=40$. $T F X=0 . T F Y=0 . T F Z=40$ $T F X=0 . T E Y=0 . T F Z=40$ $T F X=0 . T F Y=0$. TFZ $=40$. $T F X=0 . T F Y=0, T F Z=40$ $T F X=0 . T F Y=0 . T F Z=40$ $T F X=0 . T F Y=0 . T E Z=40$ $T F X=0, T E Y=0$, TEZ $=40$. $T F X=0 . T F Y=0 . T F Z=40$. $T E X=0 . T F Y=0 . T F Z=40$ $T F X=0 . T F Y=0 . T F Z=40$ $T F X=0$. TFY $=0$. TFZ $=40$ $T E X=0, T E Y=0, T F Z=40$ $T F X=0 . T F Y=0, T F Z=40$ $T F X=0, T E Y=0 . T F Z=40$. $T F X=0 ， T E Y=0 ， T F Z=40$ $T F X=0$. TEY $=0$. TFZ=40.

$T F X=0 . T F Y=0 . T F Z=40$. $T F X=0 . T Y Y=0 . T F Z=40$. $T F X=0, T E Y=0, T E Z=40$ $T E X=0 . T E Y=0 . T E Z=40$. $T E X=0 . T E Y=0 . T E Z=40$. $T E X=0 . T F Y=0 . T F Z=40$ $T E X=0$. TEY $=0$. TEZ $=40$. $T F X=0$. TFY $=0$. TEZ $=40$. $T E X=0 . T E Y=0 . T F Z=40$. TFX=0. TEY $=0$. TFZ=40. $T E X=0 . T F Y=0 . T E Z=40$ TFX=0. TEY $=0$. TFZ=40. $T F X=0 . T E Y=0$. TFZ=40. $T E X=0 . T E Y=0 . T F Z=40$ $T E X=0 . T F Y=0 . T E Z=40$. $T E X=0 . T E Y=0 . T E Z=40$ $T F X=0 . T E Y=0 . T E Z=40$. $T F X=0, T F Y=0 . T F Z=40$. $T F X=0 . T E Y=0 . T E Z=40$ $T E X=0 . T F Y=0$. TEZ $=40$ TEX $=0$, TEY $=0$. TEZ $=40$.

$T F X=0 . T F Y=0 . T F Z=40$ $T E X=0 . T F Y=0, T E Z=40$ $T E X=0 . T E Y=0 . T E Z=40$. $T F X=0 . T F Y=0 . T F Z=40$. $T E X=0 . T E Y=0 . T F Z=40$ $T F X=0 . T E Y=0 . T F Z=40$ $T E X=0, T E Y=0 . T E Z=40$ $T F X=0 . T F Y=0, T F Z=40$ $T F X=0 . T F Y=0, T F Z=40$ $T F X=0 . T F Y=0 . T F Z=40$ TEX $=0$. TFY $=0$. TFZ $=40$. $T F X=0, T F Y=0 . T F Z=40$.
LNU $=343$

$L N U=344$ $L N U=345$ LNU 346 INU $=347$ LNU $=348$ LNU $=349$ INU 350 INU $=351$ INU $=352$ LNU 353 $I N U=354$ LNU $=355$ LNU=356 LNU 357

LNU $=358$ INUM 359 INU $=360$ INU $=361$ LNUT=362 LNU $=363$ $I N U=364$ INU $=365$ INU=366 INU $=367$ LNV=368 LNU 369 $I N U=370$ LNU $=371$ INU 372 INU 373 LNU $=374$ INU $=375$ พNU=376 LNU $=377$ INU $=378$

LNO=379 LrU $=380$ LNU $=381$ LNU 382 INU 383 LNU $=384$ LNU $=385$ LNU 386 INU 387 LNU 388 LNU 389 LNU 390 WNU 391 LNU $=392$ LNU 393 LNU $=394$ LNU $=395$ LNU $=396$ LNU $=397$ LNU $=398$ INU $=399$

$\operatorname{LN}=400$ INU 401 INU 402 $L N U=403$ $L N U=404$ LNU $=405$ LNU 406 LNU $=407$ LNU $=408$ INU $=409$ LNU 410 LNU $=411$ 


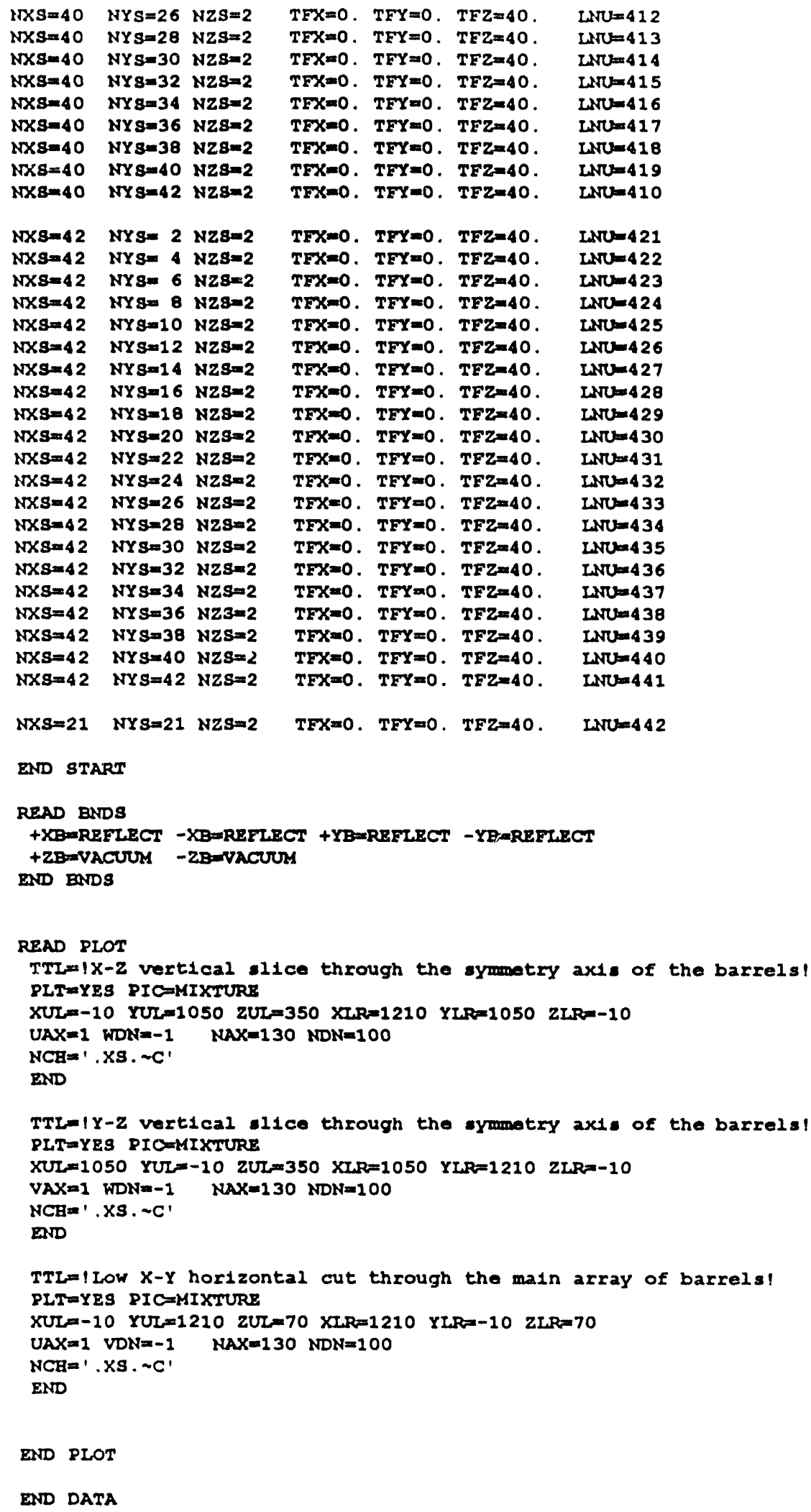

END START

READ BNDS

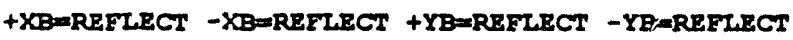

$+\mathrm{ZB}=\mathrm{VACUTM}-\mathrm{ZB}$-VACUTM

END ENDS

READ PLOT

TTI $1 X-2$ vertical slce through the symatry axd of the barrels! PLT=YES PICMIXIURE

$X U L=-10 \quad Y U L=1050 \quad Z U L=350 \quad X L R=1210 \quad Y L R=1050 \quad Z L R=-10$

$U A X=1$ WDN $=-1 \quad N A X=130 \quad N D N=100$

$\mathrm{NCA}={ }^{\prime} \cdot \mathrm{XS}, \sim \mathrm{C}^{\prime}$

BND

TTL-|Y-Z vertical al1ce through the aymatry axis of the barrels! PLTEYES PICMIXTUR:

$X U I=1050 \quad Y U L=-10 \quad Z U I=350 \quad X I R=1050 \quad Y L R=1210 \quad Z L R=-10$

$V A X=1$ WDN $=-1 \quad N A X=130$ NDN $=100$

NCE $=1 \times 9, \sim C^{\prime}$

BND

TTL=lLow $X-Y$ horizontal cut through the main array of barrels! PLTEYES PICMIXTURE

$X U L=-10 \quad Y U L=1210 \quad Z U L=70 \quad X L R=1210 \quad Y L R=-10 \quad Z L R=70$

$U A X=1$ VDN $=-1 \quad N A X=130 \quad N D N=100$

$\mathrm{NCH}={ }^{\prime} . \mathrm{XS} \cdot \sim \mathrm{C}^{\prime}$

END

END PIOT

END DATA

END 


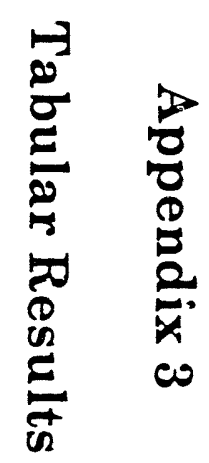




\section{Table CON-1}

\section{K-effective Vs Concrete Type}

5 Gallon Steel Drums filled with Uranyl Fluoride - Water Mixture $30 \mathrm{~cm}$ Pitch 5\% Enrichment $1.5 \mathrm{gm}$ of $U / \mathrm{cc}$ of solution

$\begin{array}{lcc}\begin{array}{c}\text { KENO V.a Standard } \\ \text { Concrete Type }\end{array} & \begin{array}{c}\text { K-effective } \\ \text { mean value }\end{array} & \begin{array}{c}\text { 1 Sigma } \\ \text { value }\end{array} \\ \text { OR-concrete } & 1.22036 & 0.00197 \\ \text { RF-concrete } & 1.21440 & 0.00184 \\ \text { MG-concrete } & 1.22901 & 0.00185 \\ \text { REG-concrete } & 1.21530 & 0.00198\end{array}$

\section{Table Con-2}

K-effective Vs Concrete Type

5 Gallon Steel Drums filled with Uranyl Fluoride - Water Mixture $100 \mathrm{~cm}$ Pitch $5 \%$ Enrichment $1.5 \mathrm{gm}$ of $U / \mathrm{cc}$ of solution

$\begin{array}{ccc}\begin{array}{c}\text { KENO V.a Standard } \\ \text { Concrete Type }\end{array} & \begin{array}{c}\text { K-effective } \\ \text { mean value }\end{array} & \begin{array}{c}\text { 1 Sigma } \\ \text { value }\end{array} \\ \text { OR-concrete } & 0.85349 & 0.00221 \\ \text { RF-concrete } & 0.84916 & 0.00211 \\ \text { MG-concrete } & 0.85674 & 0.00218 \\ \text { REG-concrete } & 0.84921 & 0.00220\end{array}$


Table MD-1

K-effective Vs Mist Density

Infinite Array of 5 Gallon Waste Containers

$1.5 \mathrm{Gms} \mathrm{U/cc} \mathrm{of} \mathrm{UO}_{2} \mathrm{~F}_{2}-\mathrm{H}_{2} \mathrm{O} \quad 5 \%$ enrichment

$30 \mathrm{~cm}$ Pitch

Mist Density

Grams H2O/cc Air-Water Mix

1.e-0

1.e-1

1.e-1

1.e-3

1.e-4

1.e-5

1.e-6

K-effective
mean value
1.14477
1.22690
1.22141
1.22123
1.21656
1.21686
1.22375

1 Sigma

value

0.00280

0.00268

0.00266

0.00267

0.00266

0.00280

0.00253

Table MD-2

K-effective Vs Mist Density

Infinite Array of 5 Gallon Waste Containers

$1.5 \mathrm{Gms} \mathrm{U} / \mathrm{cc}$ of $\mathrm{UO}_{2} \mathrm{~F}_{2}-\mathrm{H}_{2} \mathrm{O} \quad 5 \%$ enrichment $50 \mathrm{~cm}$ Pitch

Mist Density

Grams H2O/cc Air-Water Mix

1.e-0

1.e-1

1.e-1

1.e-3

1.e-4

1.e-5

1.e-6

$\begin{array}{cc}\text { K-effective } & \begin{array}{c}\text { Sigma } \\ \text { value }\end{array} \\ 0.91668 & 0.00323 \\ 1.00063 & 0.00277 \\ 1.03335 & 0.00322 \\ 1.03877 & 0.00267 \\ 1.03615 & 0.00314 \\ 1.02961 & 0.00301 \\ 1.02734 & 0.00301\end{array}$




\section{Table MD-3}

\section{K-effective Vs Mist Density \\ Infinite Array of 5 Gallon Waste Containers \\ $1.5 \mathrm{Gms} \mathrm{U/cc}$ of $\mathrm{UO}_{2} \mathrm{~F}_{2}-\mathrm{H}_{2} \mathrm{O} \quad 5 \%$ enrichment \\ $75 \mathrm{~cm}$ Pitch}

$\begin{array}{ccc}\begin{array}{c}\text { Mist Density } \\ \text { Grams H2O/cc Air-Water Mix }\end{array} & \begin{array}{c}\text { K-effective } \\ \text { mean value }\end{array} & \begin{array}{c}\text { 1 Sigma } \\ \text { value }\end{array} \\ \text { 1.e-0 } & 0.90772 & 0.00330 \\ 1 . e-1 & 0.86407 & 0.00345 \\ 1 . e-1 & 0.91783 & 0.00289 \\ 1 . e-3 & 0.91086 & 0.00320 \\ 1 . e-4 & 0.91783 & 0.00289 \\ 1 . e-5 & 0.90966 & 0.00301 \\ 1 . e-6 & 0.91470 & 0.00323\end{array}$

Table MD-4

K-ffective Vs Mist Density

Infinite Array of 5 Gallon Waste Containers

$1.5 \mathrm{Gms} \mathrm{U} / \mathrm{cc}$ of $\mathrm{UO}_{2} \mathrm{~F}_{2}-\mathrm{H}_{2} \mathrm{O} \quad 5 \%$ enrichment $100 \mathrm{~cm}$ Pitch

$\begin{array}{ccc}\begin{array}{c}\text { Mist Density } \\ \text { Grams H2O/ce Air-Water Mix }\end{array} & \begin{array}{c}\text { K-effective } \\ \text { mean value }\end{array} & \begin{array}{c}\text { 1 Sigma } \\ \text { value }\end{array} \\ \text { 1.e-0 } & 0.90989 & 0.00344 \\ 1 . e-1 & 0.83192 & 0.00378 \\ 1 . e-1 & 0.84604 & 0.00348 \\ 1 . e-3 & 0.84647 & 0.00329 \\ 1 . e-4 & 0.85285 & 0.00317 \\ 1 . e-5 & 0.85060 & 0.00327 \\ \text { 1.e-6 } & 0.85333 & 0.00314\end{array}$


Table AA

K-effective Vs Uranium Concentration

5 Gallon Steel Drums filled with $\mathrm{UF}_{4}$ - Oil Mixture

MG type concrete $\quad 10^{-4}$ (gm water/cc of air) mist density $30 \mathrm{~cm}$ Pitch 5\% Enrichment

$\begin{array}{ccc}\begin{array}{c}\text { Uranium Concentration } \\ \text { Grams U/cc Solution }\end{array} & \begin{array}{c}\text { K-effective } \\ \text { mean value } \\ 0.61623\end{array} & \begin{array}{c}\text { 1 Sigma } \\ \text { value }\end{array} \\ 0.2 & 0.00147 \\ 0.3 & 0.89364 & 0.00170 \\ 0.4 & 0.97991 & 0.00194 \\ 0.5 & 1.04325 & 0.00207 \\ 0.6 & 1.09347 & 0.00200 \\ 0.7 & 1.13489 & 0.00198 \\ 0.8 & 1.15998 & 0.00234 \\ 0.9 & 1.18604 & 0.00230 \\ 1.0 & 1.25158 & 0.00255 \\ 1.5 & 1.26092 & 0.00268 \\ 2.0 & 1.22632 & 0.00297 \\ 2.5 & 1.17814 & 0.00290 \\ 3.0 & 1.00422 & 0.00265 \\ 4.0 & 0.72161 & 0.00220\end{array}$

Table UA

K-effective Vs Uranium Concentration

5 Gallon Steel Drums filled with $\mathrm{UF}_{4}$ - Oil Mixture

MG type concrete $\quad 10^{-4}$ (gm water/cc of air) mist density $30 \mathrm{~cm}$ Pitch 2\% Enrichment

$\begin{array}{ccc}\begin{array}{c}\text { Uranium Concentration } \\ \text { Grams U/cc Solution }\end{array} & \begin{array}{c}\text { K-effective } \\ \text { mean value } \\ 0.31127\end{array} & \begin{array}{c}\text { Sigma } \\ \text { value }\end{array} \\ 0.2 & 0.42446 & 0.00099 \\ 0.3 & 0.51827 & 0.00110 \\ 0.4 & 0.59491 & 0.00110 \\ 0.5 & 0.65957 & 0.00174 \\ 0.6 & 0.71405 & 0.00155 \\ 0.7 & 0.76600 & 0.00155 \\ 0.8 & 0.80416 & 0.00169 \\ 0.9 & 0.84010 & 0.00175 \\ 1.0 & 0.95430 & 0.00187 \\ 1.5 & 1.00104 & 0.00215 \\ 2.0 & 1.01293 & 0.00236 \\ 2.5 & 0.99583 & 0.00230 \\ 3.0 & 0.85390 & 0.00230 \\ 4.0 & 0.56283 & 0.00216 \\ 5.0 & & \end{array}$


Table AB

K-effective Vs Uranium Concentration

5 Gallon Steel Drums filled with $\mathrm{UF}_{4}$ - Oil Mixture

MG type concrete $\quad 10^{-4}$ (gm water/cc of air) mist density

$50 \mathrm{~cm}$ Pitch 5\% Enrichment

$\begin{array}{ccc}\begin{array}{c}\text { Uranium Concentration } \\ \text { Grams U/cc Solution }\end{array} & \begin{array}{c}\text { K-effective } \\ \text { mean value }\end{array} & \begin{array}{c}\text { 1 Sigma } \\ \text { value }\end{array} \\ 0.2 & 0.53573 & 0.00182 \\ 0.3 & 0.67677 & 0.00210 \\ 0.4 & 0.78197 & 0.00216 \\ 0.5 & 0.85325 & 0.00222 \\ 0.6 & 0.91345 & 0.00253 \\ 0.7 & 0.95573 & 0.00260 \\ 0.8 & 0.99459 & 0.00273 \\ 0.9 & 1.01533 & 0.00278 \\ 1.0 & 1.04357 & 0.00250 \\ 1.5 & 1.08574 & 0.00294 \\ 2.0 & 1.08954 & 0.00295 \\ 2.5 & 1.04899 & 0.00296 \\ 3.0 & 0.99436 & 0.00281 \\ 4.0 & 0.83098 & 0.00298 \\ 5.0 & 0.62693 & 0.00266\end{array}$

Table UB

K-effective Vs Uranium Concentration

5 Gallon Steel Drums filled with UF 4 - Oil Mixture

MG type concrete $10^{-4}$ (gm water/cc of air) mist density $50 \mathrm{~cm}$ Pitch 2\% Enrichment

$\begin{array}{ccc}\begin{array}{c}\text { Uranium Concentration } \\ \text { Grams U/cc Solution }\end{array} & \begin{array}{c}\text { Keffective } \\ \text { mean value }\end{array} & \begin{array}{c}\text { 1 Sigma } \\ \text { value }\end{array} \\ 0.2 & 0.26950 & 0.00103 \\ 0.3 & 0.36835 & 0.00123 \\ 0.4 & 0.44692 & 0.00144 \\ 0.5 & 0.51298 & 0.00147 \\ 0.6 & 0.56713 & 0.00179 \\ 0.7 & 0.61870 & 0.00187 \\ 0.8 & 0.65867 & 0.00209 \\ 0.9 & 0.69077 & 0.00215 \\ 1.0 & 0.72357 & 0.00226 \\ 1.5 & 0.82014 & 0.00230 \\ 2.0 & 0.85712 & 0.00247 \\ 2.5 & 0.84939 & 0.00228 \\ 3.0 & 0.82410 & 0.00256 \\ 4.0 & 0.69023 & 0.00223 \\ 5.0 & 0.50230 & 0.00227\end{array}$


Table AD

K-effective Vs Uranium Concentration

5 Gallon Steel Drums filled with $\mathrm{UF}_{4}$ - Oil Mixture

MG type concrete $\quad 10^{-4}$ (gm water/cc of air) mist density

$75 \mathrm{~cm}$ Pitch $5 \%$ Enrichment

$\begin{array}{ccc}\begin{array}{c}\text { Uranium Concentration } \\ \text { Grams U/cc Solution }\end{array} & \begin{array}{c}\text { Keffective } \\ \text { mean value }\end{array} & \begin{array}{c}\text { 1 Sigma } \\ \text { value }\end{array} \\ 0.2 & 0.49651 & 0.00148 \\ 0.3 & 0.62870 & 0.00178 \\ 0.4 & 0.72153 & 0.00220 \\ 0.5 & 0.78866 & 0.00243 \\ 0.6 & 0.84007 & 0.00237 \\ 0.7 & 0.87797 & 0.00267 \\ 0.8 & 0.91229 & 0.00271 \\ 0.9 & 0.93650 & 0.00289 \\ 1.0 & 0.95061 & 0.00293 \\ 1.5 & 0.98397 & 0.00315 \\ 2.0 & 0.96923 & 0.00338 \\ 2.5 & 0.91686 & 0.00317 \\ 3.0 & 0.86488 & 0.00300 \\ 4.0 & 0.69285 & 0.00273 \\ 5.0 & 0.50830 & 0.00224\end{array}$

Table UD

K-effective Vs Uranium Concentration

5 Gallon Steel Drums filled with $\mathrm{UF}_{4}$ - Oil Mixture

MG type concrete $10^{-4}$ (gm water/cc of air) mist density $75 \mathrm{~cm}$ Pitch $2 \%$ Enrichment

$\begin{array}{ccc}\begin{array}{c}\text { Uranium Concentration } \\ \text { Grams U/cc Solution }\end{array} & \begin{array}{c}\text { K-effective } \\ \text { mean value } \\ 0.24844\end{array} & \begin{array}{c}\text { 1 Sigma } \\ \text { value }\end{array} \\ 0.2 & 0.00076 \\ 0.3 & 0.33910 & 0.00103 \\ 0.4 & 0.41435 & 0.00123 \\ 0.5 & 0.47227 & 0.00138 \\ 0.6 & 0.52043 & 0.00166 \\ 0.7 & 0.56789 & 0.00163 \\ 0.8 & 0.60146 & 0.00185 \\ 0.9 & 0.63242 & 0.00185 \\ 1.0 & 0.66464 & 0.00210 \\ 1.5 & 0.74480 & 0.00239 \\ 2.0 & 0.76191 & 0.00246 \\ 2.5 & 0.74647 & 0.00252 \\ 3.0 & 0.70915 & 0.00259 \\ 4.0 & 0.56199 & 0.00228 \\ 5.0 & 0.40176 & 0.00198\end{array}$


Table AE

K-effective Vs Uranium Concentration

5 Gallon Steel Drums filled with $\mathrm{UF}_{4}$ - Oil Mixture

MG type concrete $\quad 10^{-4}$ (gm water/cc of air) mist density $100 \mathrm{~cm}$ Pitch 5\% Enrichment

$\begin{array}{ccc}\begin{array}{c}\text { Uranium Concentration } \\ \text { Grams U/cc Solution }\end{array} & \begin{array}{c}\text { K-effective } \\ \text { mean value }\end{array} & \begin{array}{c}\text { Sigma } \\ \text { value }\end{array} \\ 0.2 & 0.47671 & 0.00172 \\ 0.3 & 0.60160 & 0.00248 \\ 0.4 & 0.69619 & 0.00234 \\ 0.5 & 0.76445 & 0.00254 \\ 0.6 & 0.81404 & 0.00314 \\ 0.7 & 0.85061 & 0.00267 \\ 0.8 & 0.87432 & 0.00297 \\ 0.9 & 0.89448 & 0.00296 \\ 1.0 & 0.91293 & 0.00308 \\ 1.5 & 0.92905 & 0.00318 \\ 2.0 & 0.91755 & 0.00319 \\ 2.5 & 0.86556 & 0.00297 \\ 3.0 & 0.78939 & 0.00312 \\ 4.0 & 0.60935 & 0.00332 \\ 5.0 & 0.40941 & 0.00228\end{array}$

Table UE

K-ffective Vs Uranium Concentration

5 Gallon Steel Drums filled with UF 4 - Oil Mixture

MG type concrete $10^{-4}$ (gm water/cc of air) mist density $100 \mathrm{~cm}$ Pitch 2\% Enrichment

$\begin{array}{ccc}\begin{array}{c}\text { Uranium Concentration } \\ \text { Grams U/cc Solution }\end{array} & \begin{array}{c}\text { Keffective } \\ \text { mean value } \\ 0.2\end{array} & \begin{array}{c}\text { 1 Sigma } \\ \text { value }\end{array} \\ 0.3 & 0.24010 & 0.00114 \\ 0.4 & 0.32900 & 0.00144 \\ 0.5 & 0.39849 & 0.00163 \\ 0.6 & 0.45609 & 0.00177 \\ 0.7 & 0.50418 & 0.00194 \\ 0.8 & 0.54511 & 0.00231 \\ 0.9 & 0.58381 & 0.00209 \\ 1.0 & 0.60636 & 0.00232 \\ 1.5 & 0.63250 & 0.00209 \\ 2.0 & 0.70519 & 0.00254 \\ 2.5 & 0.71647 & 0.00262 \\ 3.0 & 0.69349 & 0.00267 \\ 4.0 & 0.64989 & 0.00265 \\ 5.0 & 0.49275 & 0.00260 \\ & 0.31987 & 0.00210\end{array}$


Table AF

Keffective Vs Uranium Concentration

5 Gallon Steel Drums filled with $\mathrm{UF}_{4}$ - Oil Mixture

MG type concrete $\quad 10^{-4}$ (gm water/cc of air) mist density $150 \mathrm{~cm}$ Pitch 5\% Enrichment

$\begin{array}{ccc}\begin{array}{c}\text { Uranium Concentration } \\ \text { Grams U/cc Solution }\end{array} & \begin{array}{c}\text { K-effective } \\ \text { mean value } \\ 0.46738\end{array} & \begin{array}{c}\text { 1 Sigma } \\ \text { value }\end{array} \\ 0.2 & 0.58446 & 0.00161 \\ 0.3 & 0.67848 & 0.00192 \\ 0.4 & 0.73161 & 0.00229 \\ 0.5 & 0.77802 & 0.00259 \\ 0.6 & 0.82424 & 0.00284 \\ 0.7 & 0.84975 & 0.00297 \\ 0.8 & 0.86835 & 0.00197 \\ 0.9 & 0.88384 & 0.00314 \\ 1.0 & 0.89717 & 0.00306 \\ 1.5 & 0.87022 & 0.00295 \\ 2.0 & 0.81094 & 0.00309 \\ 2.5 & 0.73694 & 0.00329 \\ 3.0 & 0.52788 & 0.00237 \\ 4.0 & 0.30787 & 0.00168\end{array}$

Table UF

K-effective Vs Uranium Concentration

5 Gallon Steel Drums filled with UF 4 - Oil Mixture

MG type concrete $10^{-4}$ (gm water/cc of air) mist density $150 \mathrm{~cm}$ Pitch 2\% Enrichment

$\begin{array}{ccc}\begin{array}{c}\text { Uranium Concentration } \\ \text { Grams U/cc Solution }\end{array} & \begin{array}{c}\text { K-effective } \\ \text { mean value }\end{array} & \begin{array}{c}\text { 1 Sigma } \\ \text { value }\end{array} \\ 0.2 & 0.23434 & 0.00075 \\ 0.3 & 0.31884 & 0.00105 \\ 0.4 & 0.38847 & 0.00123 \\ 0.5 & 0.44362 & 0.00154 \\ 0.6 & 0.48930 & 0.00156 \\ 0.7 & 0.53071 & 0.00185 \\ 0.8 & 0.56292 & 0.00192 \\ 0.9 & 0.58488 & 0.00192 \\ 1.0 & 0.61341 & 0.00213 \\ 1.5 & 0.67494 & 0.00227 \\ 2.0 & 0.68598 & 0.00265 \\ 2.5 & 0.65501 & 0.00278 \\ 3.0 & 0.60475 & 0.00236 \\ 4.0 & 0.42250 & 0.00211 \\ 5.0 & 0.23784 & 0.00145\end{array}$


Table DA

K-effective Vs Uranium Concentration

5 Gallon Steel Drums filled with $\mathrm{UO}_{2} \mathrm{~F}_{2}$ - Water Mixture MG type concrete $10^{-4}$ (gm water/cc of air) mist density $30 \mathrm{~cm}$ Pitch $5 \%$ Enrichment

$\begin{array}{ccc}\begin{array}{c}\text { Uranium Concentration } \\ \text { Grams U/cc Solution }\end{array} & \begin{array}{c}\text { Keffective } \\ \text { mean value }\end{array} & \begin{array}{c}\text { 1 Sigma } \\ \text { value }\end{array} \\ 0.2 & 0.68242 & 0.00184 \\ 0.3 & 0.83369 & 0.00190 \\ 0.4 & 0.93676 & 0.00202 \\ 0.5 & 1.01706 & 0.00211 \\ 0.6 & 1.07169 & 0.00211 \\ 0.7 & 1.11195 & 0.00216 \\ 0.8 & 1.13866 & 0.00242 \\ 0.9 & 1.16538 & 0.00261 \\ 1.0 & 1.18639 & 0.00245 \\ 1.5 & 1.22999 & 0.00257 \\ 2.0 & 1.22528 & 0.00266 \\ 2.5 & 1.21163 & 0.00292 \\ 3.0 & 1.17589 & 0.00295 \\ 4.0 & 1.12269 & 0.00261 \\ 4.5 & 0.97011 & 0.00266\end{array}$

Table XA

K-effective Vs Uranium Concentration

5 Gallon Steel Drums filled with $\mathrm{UO}_{2} \mathrm{~F}_{2}$ - Water Mixture MG type concrete $10^{-4}$ (gm water/cc of air) mist density $30 \mathrm{~cm}$ Pitch $2 \%$ Enrichment

$\begin{array}{ccc}\begin{array}{c}\text { Uranium Concentration } \\ \text { Grams U/cc Solution }\end{array} & \begin{array}{c}\text { K-effective } \\ \text { mean value }\end{array} & \begin{array}{c}\text { 1 Sigma } \\ \text { value }\end{array} \\ 0.2 & 0.35863 & 0.00102 \\ 0.3 & 0.47422 & 0.00125 \\ 0.4 & 0.56631 & 0.00144 \\ 0.5 & 0.64327 & 0.00164 \\ 0.6 & 0.70379 & 0.00183 \\ 0.7 & 0.74997 & 0.00187 \\ 0.8 & 0.79382 & 0.00184 \\ 0.9 & 0.83537 & 0.00174 \\ 1.0 & 0.86050 & 0.00187 \\ 1.5 & 0.95966 & 0.00208 \\ 2.0 & 0.99361 & 0.00210 \\ 2.5 & 1.00180 & 0.00225 \\ 3.0 & 0.99422 & 0.00236 \\ 4.0 & 0.95680 & 0.00231 \\ 4.5 & 0.82418 & 0.00237\end{array}$


Table DB

K-effective Vs Uranium Concentration

5 Gallon Steel Drums filled with $\mathrm{UO}_{2} \mathrm{~F}_{2}$ - Water Mixture MG type concrete $\quad 10^{-4}$ (gm water/cc of air) mist density $50 \mathrm{~cm}$ Pitch $5 \%$ Enrichment

$\begin{array}{ccc}\begin{array}{c}\text { Uranium Concentration } \\ \text { Grams U/cc Solution }\end{array} & \begin{array}{c}\text { K-effective } \\ \text { mean value }\end{array} & \begin{array}{c}\text { 1 Sigma } \\ \text { value }\end{array} \\ 0.2 & 0.56337 & 0.00206 \\ 0.3 & 0.69350 & 0.00230 \\ 0.4 & 0.78132 & 0.00246 \\ 0.5 & 0.85279 & 0.00246 \\ 0.6 & 0.89524 & 0.00270 \\ 0.7 & 0.93174 & 0.00262 \\ 0.8 & 0.96329 & 0.00270 \\ 0.9 & 0.98804 & 0.00261 \\ 1.0 & 1.00489 & 0.00256 \\ 1.5 & 1.04363 & 0.00289 \\ 2.0 & 1.04262 & 0.00306 \\ 2.5 & 1.02380 & 0.00293 \\ 3.0 & 0.98766 & 0.00303 \\ 4.0 & 0.95104 & 0.00270 \\ 4.5 & 0.80826 & 0.00292\end{array}$

Table XB

K-effective Vs Uranium Concentration

5 Gallon Steel Drums filled with $\mathrm{UO}_{2} \mathrm{~F}_{2}$ - Water Mixture MG type concrete $10^{-4}$ (gm water/cc of air) mist density $50 \mathrm{~cm}$ Pitch $2 \%$ Enrichment

$\begin{array}{ccc}\begin{array}{c}\text { Uranium Concentration } \\ \text { Grams U/cc Solution }\end{array} & \begin{array}{c}\text { K-effective } \\ \text { mean value }\end{array} & \begin{array}{c}\text { 1 Sigma } \\ \text { value }\end{array} \\ 0.2 & 0.29497 & 0.00127 \\ 0.3 & 0.38536 & 0.00131 \\ 0.4 & 0.46471 & 0.00180 \\ 0.5 & 0.53340 & 0.00196 \\ 0.6 & 0.58348 & 0.00212 \\ 0.7 & 0.62743 & 0.00222 \\ 0.8 & 0.66502 & 0.00208 \\ 0.9 & 0.69532 & 0.00213 \\ 1.0 & 0.71597 & 0.00222 \\ 1.5 & 0.79335 & 0.00234 \\ 2.0 & 0.83482 & 0.00241 \\ 2.5 & 0.83590 & 0.00234 \\ 3.0 & 0.82699 & 0.00258 \\ 4.0 & 0.80405 & 0.00226 \\ 4.5 & 0.67105 & 0.00261\end{array}$


Table DD

K-effective Vs Uranium Concentration

5 Gallon Steel Drums filled with $\mathrm{UO}_{2} \mathrm{~F}_{2}$ - Water Mixture

MG type concrete $10^{-4}$ (gm water/cc of air) mist density $75 \mathrm{~cm}$ Pitch $5 \%$ Enrichment

$\begin{array}{ccc}\begin{array}{c}\text { Uranium Concentration } \\ \text { Grams U/cc Solution }\end{array} & \begin{array}{c}\text { K-effective } \\ \text { mean value }\end{array} & \begin{array}{c}\text { 1 Sigma } \\ \text { value }\end{array} \\ 0.2 & 0.49944 & 0.00154 \\ 0.3 & 0.61393 & 0.00202 \\ 0.4 & 0.69837 & 0.00226 \\ 0.5 & 0.75629 & 0.00260 \\ 0.6 & 0.80149 & 0.00281 \\ 0.7 & 0.83184 & 0.00295 \\ 0.8 & 0.85162 & 0.00289 \\ 0.8 & 0.87316 & 0.00302 \\ 1.0 & 0.88599 & 0.00308 \\ 1.5 & 0.92723 & 0.00301 \\ 2.0 & 0.92003 & 0.00300 \\ 2.5 & 0.89457 & 0.00334 \\ 3.0 & 0.86429 & 0.00307 \\ 4.0 & 0.82425 & 0.00300 \\ 4.5 & 0.66677 & 0.00270\end{array}$

Table XD

K-effective Vs Uranium Concentration

5 Gallon Steel Drums filled with $\mathrm{UO}_{2} \mathrm{~F}_{2}$ - Water Mixture MG type concrete $10^{-4}$ (gm water/cc of air) mist density $75 \mathrm{~cm}$ Pitch 2\% Enrichment

$\begin{array}{ccc}\begin{array}{c}\text { Uranium Concentration } \\ \text { Grams U/cc Solution }\end{array} & \begin{array}{c}\text { Keffective } \\ \text { mean value }\end{array} & \begin{array}{c}\text { 1 Sigma } \\ \text { value }\end{array} \\ 0.2 & 0.25947 & 0.00091 \\ 0.3 & 0.34473 & 0.00119 \\ 0.4 & 0.41231 & 0.00139 \\ 0.5 & 0.46822 & 0.00150 \\ 0.6 & 0.51471 & 0.00177 \\ 0.7 & 0.55599 & 0.00185 \\ 0.8 & 0.58289 & 0.00193 \\ 0.9 & 0.61135 & 0.00197 \\ 1.0 & 0.62784 & 0.00213 \\ 1.5 & 0.70384 & 0.00224 \\ 2.0 & 0.72244 & 0.00260 \\ 2.5 & 0.71920 & 0.00245 \\ 3.0 & 0.70468 & 0.00241 \\ 4.0 & 0.67919 & 0.00246 \\ 4.5 & 0.54608 & 0.00242\end{array}$


Table DE

Keffective Vs Uranium Concentration

5 Gallon Steel Drums filled with $\mathrm{UO}_{2} \mathrm{~F}_{2}$ - Water Mixture MG type concrete $10^{-4}$ (gm water/cc of air) mist density $100 \mathrm{~cm}$ Pitch $5 \%$ Enrichment

\begin{tabular}{|c|c|c|}
\hline $\begin{array}{l}\text { Uranium Concentration } \\
\text { Grams U/cc Solution }\end{array}$ & $\begin{array}{l}\text { K-effective } \\
\text { mean value }\end{array}$ & $\begin{array}{c}1 \text { Sigma } \\
\text { value } \\
0.00184\end{array}$ \\
\hline 0.2 & $\begin{array}{l}0.47233 \\
0.57995\end{array}$ & $\begin{array}{l}0.00184 \\
0.00192\end{array}$ \\
\hline $\begin{array}{l}0.3 \\
0.4\end{array}$ & 0.65891 & 0.00250 \\
\hline $\begin{array}{l}0.4 \\
0.5\end{array}$ & 0.71243 & 0.00251 \\
\hline 0.6 & 0.75442 & 0.00247 \\
\hline 0.7 & 0.77588 & 0.00277 \\
\hline 0.8 & 0.80775 & 0.00276 \\
\hline 0.9 & 0.81824 & 0.00316 \\
\hline 1.0 & 0.83668 & 0.00312 \\
\hline 1.5 & 0.86329 & 0.00322 \\
\hline 2.0 & 0.85027 & 0.00333 \\
\hline 2.5 & 0.82776 & 0.00314 \\
\hline 3.0 & 0.78644 & 0.00290 \\
\hline 4.0 & 0.76143 & 0.00293 \\
\hline 4.5 & 0.57866 & 0.00259 \\
\hline
\end{tabular}

Table XE

K-effective Vs Uranium Concentration

5 Gallon Steel Drums filled with $\mathrm{UO}_{2} \mathrm{~F}_{2}$ - Water Mixture MG type concrete $10^{-4}$ (gm water/cc of air) mist density $100 \mathrm{~cm}$ Pitch 2\% Enrichment

$\begin{array}{ccc}\begin{array}{c}\text { Uranium Concentration } \\ \text { Grams U/cc Solution }\end{array} & \begin{array}{c}\text { Keffective } \\ \text { mean value }\end{array} & \begin{array}{c}\text { 1 Sigma } \\ \text { value }\end{array} \\ 0.2 & 0.24652 & 0.00082 \\ 0.3 & 0.32586 & 0.00130 \\ 0.4 & 0.38863 & 0.00140 \\ 0.5 & 0.44239 & 0.00157 \\ 0.6 & 0.48394 & 0.00184 \\ 0.7 & 0.51784 & 0.00182 \\ 0.8 & 0.54880 & 0.00205 \\ 0.9 & 0.57426 & 0.00212 \\ 1.0 & 0.59392 & 0.00223 \\ 1.5 & 0.65621 & 0.00246 \\ 2.0 & 0.67353 & 0.00265 \\ 2.5 & 0.67063 & 0.00268 \\ 3.0 & 0.65418 & 0.00256 \\ 4.0 & 0.62728 & 0.00244 \\ 4.5 & 0.46491 & 0.00218\end{array}$


Table DF

K-ffective Vs Uranium Concentration

5 Gallon Steel Drums filled with $\mathrm{UO}_{2} \mathrm{~F}_{2}$ - Water Mixture MG type concrete $10^{-4}$ (gm water/cc of air) mist density $150 \mathrm{~cm}$ Pitch $5 \%$ Enrichment

$\begin{array}{ccc}\begin{array}{c}\text { Uranium Concentration } \\ \text { Grams U/cc Solution }\end{array} & \begin{array}{c}\text { Keffective } \\ \text { mean value } \\ 0.45250\end{array} & \begin{array}{c}\text { Sigma } \\ \text { value }\end{array} \\ 0.2 & 0.00176 \\ 0.3 & 0.55602 & 0.00200 \\ 0.4 & 0.62343 & 0.00259 \\ 0.5 & 0.68013 & 0.00250 \\ 0.6 & 0.71857 & 0.00264 \\ 0.7 & 0.74745 & 0.00275 \\ 0.8 & 0.76636 & 0.00301 \\ 0.9 & 0.78140 & 0.00324 \\ 1.0 & 0.78882 & 0.00324 \\ 1.5 & 0.81224 & 0.00356 \\ 2.0 & 0.80215 & 0.00341 \\ 2.5 & 0.77766 & 0.00323 \\ 3.0 & 0.72927 & 0.00307 \\ 4.0 & 0.70203 & 0.00316 \\ 4.5 & 0.50376 & 0.00250\end{array}$

Table XF

K-ffective Vs Uranium Concentration

5 Gallon Steel Drums filled with $\mathrm{UO}_{2} \mathrm{~F}_{2}$ - Water Mixture MG type concrete $\quad 10^{-4}$ (gm water/cc of air) mist density $150 \mathrm{~cm}$ Pitch $2 \%$ Enrichment

$\begin{array}{ccc}\begin{array}{c}\text { Uranium Concentration } \\ \text { Grams U/cc Solution }\end{array} & \begin{array}{c}\text { K-effective } \\ \text { mean value }\end{array} & \begin{array}{c}\text { 1 Sigma } \\ \text { value }\end{array} \\ 0.2 & 0.23480 & 0.00099 \\ 0.3 & 0.30999 & 0.00125 \\ 0.4 & 0.37250 & 0.00147 \\ 0.5 & 0.42318 & 0.00172 \\ 0.6 & 0.46196 & 0.00178 \\ 0.7 & 0.49688 & 0.00187 \\ 0.8 & 0.52359 & 0.00205 \\ 0.9 & 0.54475 & 0.00207 \\ 1.0 & 0.56673 & 0.00230 \\ 1.5 & 0.61831 & 0.00258 \\ 2.0 & 0.63289 & 0.00263 \\ 2.5 & 0.62873 & 0.00267 \\ 3.0 & 0.60193 & 0.00274 \\ 4.0 & 0.58092 & 0.00266 \\ 4.5 & 0.39940 & 0.00194\end{array}$


Table BB

K-effective Vs Uranium Concentration

30 Gallon Steel Drums filled with $\mathrm{UF}_{4}$ - Oil Mixture

MG type concrete $\quad 10^{-4}$ (gm water/ce of air) mist density $50 \mathrm{~cm}$ Pitch 5\% Enrichment

$\begin{array}{ccc}\begin{array}{c}\text { Uranium Concentration } \\ \text { Grams U/cc Solution }\end{array} & \begin{array}{c}\text { K-effective } \\ \text { mean value }\end{array} & \begin{array}{c}\text { Sigma } \\ \text { value }\end{array} \\ 0.2 & 0.66980 & 0.00121 \\ 0.3 & 0.84210 & 0.00140 \\ 0.4 & 0.96622 & 0.00162 \\ 0.5 & 1.05820 & 0.00167 \\ 0.6 & 1.12811 & 0.00174 \\ 0.7 & 1.17893 & 0.00199 \\ 0.8 & 1.22145 & 0.00213 \\ 0.9 & 1.24984 & 0.00193 \\ 1.0 & 1.28003 & 0.00208 \\ 1.5 & 1.34687 & 0.00236 \\ 2.0 & 1.35145 & 0.00240 \\ 2.5 & 1.32797 & 0.00239 \\ 3.0 & 1.27772 & 0.00255 \\ 4.0 & 1.09559 & 0.00243 \\ 5.0 & 0.74377 & 0.00177\end{array}$

Table VB

K-effective Vs Uranium Concentration

30 Gallon Steel Drums filled with $\mathrm{UF}_{4}$ - Oil Mixture

MG type concrete $10^{-4}$ (gm water/cc of air) mist density $50 \mathrm{~cm}$ Pitch $2 \%$ Enrichment

$\begin{array}{ccc}\begin{array}{c}\text { Uranium Concentration } \\ \text { Grams U/cc Solution }\end{array} & \begin{array}{c}\text { Keffective } \\ \text { mean value }\end{array} & \begin{array}{c}\text { 1 Sigma } \\ \text { value }\end{array} \\ 0.2 & 0.34169 & 0.00080 \\ 0.3 & 0.46349 & 0.00091 \\ 0.4 & 0.56490 & 0.00116 \\ 0.5 & 0.64775 & 0.00119 \\ 0.6 & 0.71717 & 0.00129 \\ 0.7 & 0.77800 & 0.00128 \\ 0.8 & 0.83232 & 0.00153 \\ 0.9 & 0.87620 & 0.00127 \\ 1.0 & 0.91115 & 0.00164 \\ 1.5 & 1.03649 & 0.00173 \\ 2.0 & 1.08908 & 0.00201 \\ 2.5 & 1.10870 & 0.00213 \\ 3.0 & 1.08867 & 0.00203 \\ 4.0 & 0.94118 & 0.00211 \\ 5.0 & 0.54040 & 0.00162\end{array}$


Table BD

K-effective Vs Uranium Concentration

30 Gallon Steel Drums filled with $U_{4}$ - Oil Mixture

MG type concrete $\quad 10^{-4}$ (gm water/cc of air) mist density $75 \mathrm{~cm}$ Pitch 5\% Enrichment

Uranium Concentration
Grams U/cc Solution
0.2
0.3
0.4
0.5
0.6
0.7
0.8
0.9
1.0
1.5
2.0
2.5
3.0
4.0
5.0

$\begin{array}{cc}\begin{array}{c}\text { K-effective } \\ \text { mean value }\end{array} & \begin{array}{c}\text { Sigma } \\ \text { value }\end{array} \\ 0.64496 & 0.00129 \\ 0.80768 & 0.00154 \\ 0.92486 & 0.00199 \\ 1.00875 & 0.00186 \\ 1.07669 & 0.00189 \\ 1.13060 & 0.00226 \\ 1.16433 & 0.00219 \\ 1.19629 & 0.00228 \\ 1.22125 & 0.00205 \\ 1.28176 & 0.00274 \\ 1.28254 & 0.00256 \\ 1.24840 & 0.00265 \\ 1.18570 & 0.00262 \\ 1.00883 & 0.00284 \\ 0.70541 & 0.00206\end{array}$

Table VD

K-effective Vs Uranium Concentration

30 Gallon Steel Drums filled with $\mathrm{UF}_{4}$ - Oil Mixture

MG type concrete $\quad 10^{-4}$ (gm water/cc of air) mist density $75 \mathrm{~cm}$ Pitch $2 \%$ Enrichment

$\begin{array}{ccc}\begin{array}{c}\text { Uranium Concentration } \\ \text { Grams U/cc Solution }\end{array} & \begin{array}{c}\text { K-effective } \\ \text { mean value } \\ 0.32793\end{array} & \begin{array}{c}\text { 1 Sigma } \\ \text { value }\end{array} \\ 0.2 & 0.00084 \\ 0.3 & 0.44542 & 0.00102 \\ 0.4 & 0.54223 & 0.00137 \\ 0.5 & 0.61913 & 0.00147 \\ 0.6 & 0.68498 & 0.00134 \\ 0.7 & 0.74391 & 0.00148 \\ 0.8 & 0.78971 & 0.00159 \\ 0.9 & 0.83407 & 0.00162 \\ 1.0 & 0.86754 & 0.00167 \\ 1.5 & 0.98563 & 0.00197 \\ 2.0 & 1.03011 & 0.00210 \\ 2.5 & 1.03947 & 0.00225 \\ 3.0 & 1.01310 & 0.00237 \\ 4.0 & 0.85643 & 0.00245 \\ 5.0 & 0.52911 & 0.00188\end{array}$


Table BE

K-effective Vs Uranium Concentration

30 Gallon Steel Drums filled with $\mathrm{UF}_{4}$ - Oil Mixture

MG type concrete $\quad 10^{-4}$ (gm water/cc of air) mist density

$100 \mathrm{~cm}$ Pitch $5 \%$ Enrichment

$\begin{array}{ccc}\begin{array}{c}\text { Uranium Concentration } \\ \text { Grams U/cc Solution }\end{array} & \begin{array}{c}\text { K-effective } \\ \text { mean value } \\ 0.63059\end{array} & \begin{array}{c}\text { 1 Sigma } \\ \text { value }\end{array} \\ 0.2 & 0.79155 & 0.00160 \\ 0.3 & 0.90211 & 0.00174 \\ 0.4 & 0.98628 & 0.00197 \\ 0.5 & 1.04869 & 0.00213 \\ 0.6 & 1.10202 & 0.00208 \\ 0.7 & 1.13170 & 0.00237 \\ 0.8 & 1.16161 & 0.00251 \\ 0.9 & 1.18453 & 0.00262 \\ 1.0 & 1.24248 & 0.00299 \\ 1.5 & 1.23601 & 0.00267 \\ 2.0 & 1.20121 & 0.00295 \\ 2.5 & 1.14373 & 0.00298 \\ 3.0 & 0.94936 & 0.00291 \\ 4.0 & 0.64522 & 0.00229 \\ 5.0 & & \end{array}$

Table VE

K-effective Vs Uranium Concentration

30 Gallon Steel Drums filled with $\mathrm{UF}_{4}$ - Oil Mixture

MG type concrete $\quad 10^{-4}$ (gm water/cc of air) mist density $100 \mathrm{~cm}$ Pitch 2\% Enrichment

$\begin{array}{ccc}\begin{array}{c}\text { Uranium Concentration } \\ \text { Grams U/cc Solution }\end{array} & \begin{array}{c}\text { K-effective } \\ \text { mean value } \\ 0.31996\end{array} & \begin{array}{c}\text { Sigma } \\ \text { value }\end{array} \\ 0.2 & 0.00086 \\ 0.3 & 0.53448 & 0.00112 \\ 0.4 & 0.60358 & 0.00129 \\ 0.5 & 0.66808 & 0.00149 \\ 0.6 & 0.72174 & 0.00180 \\ 0.7 & 0.76953 & 0.00156 \\ 0.8 & 0.80996 & 0.00176 \\ 0.9 & 0.84435 & 0.00183 \\ 1.0 & 0.95109 & 0.00204 \\ 1.5 & 0.99531 & 0.00246 \\ 2.0 & 0.99172 & 0.00261 \\ 2.5 & 0.96399 & 0.00246 \\ 3.0 & 0.79780 & 0.00251 \\ 4.0 & 0.49056 & 0.00199\end{array}$




\section{Table BF}

K-effective Vs Uranium Concentration

30 Gallon Steel Drums filled with UF 4 - Oil Mixture

MG type concrete $10^{-4}$ (gm water/cc of air) mist density $150 \mathrm{~cm}$ Pitch 5\% Enrichment

$\begin{array}{ccc}\begin{array}{c}\text { Uranium Concentration } \\ \text { Grams U/cc Solution }\end{array} & \begin{array}{c}\text { Keffective } \\ \text { mean value }\end{array} & \begin{array}{c}\text { 1 Sigma } \\ \text { value }\end{array} \\ 0.2 & 0.61484 & 0.00166 \\ 0.3 & 0.77151 & 0.00204 \\ 0.4 & 0.88712 & 0.00212 \\ 0.5 & 0.96308 & 0.00202 \\ 0.6 & 1.02378 & 0.00206 \\ 0.7 & 1.07662 & 0.00257 \\ 0.8 & 1.10808 & 0.00235 \\ 0.9 & 1.13641 & 0.00245 \\ 1.0 & 1.15458 & 0.00281 \\ 1.5 & 1.19481 & 0.00270 \\ 2.0 & 1.18868 & 0.00314 \\ 2.5 & 1.14771 & 0.00292 \\ 3.0 & 1.08445 & 0.00308 \\ 4.0 & 0.87038 & 0.00292 \\ 5.0 & 0.54657 & 0.00213\end{array}$

Table VF

Keffective Vs Uranium Concentration

30 Gallon Steel Drums filled with UF 4 - Oil Mixture

MG type concrete $10^{-4}$ (gm water/cc of air) mist density $150 \mathrm{~cm}$ Pitch 2\% Enrichment

$\begin{array}{ccc}\begin{array}{c}\text { Uranium Concentration } \\ \text { Grams U/cc Solution }\end{array} & \begin{array}{c}\text { Keffective } \\ \text { mean value } \\ 0.31348\end{array} & \begin{array}{c}\text { 1 Sigma } \\ \text { value }\end{array} \\ 0.2 & 0.00103 \\ 0.3 & 0.42465 & 0.00122 \\ 0.4 & 0.51641 & 0.00130 \\ 0.5 & 0.58603 & 0.00147 \\ 0.6 & 0.65525 & 0.00162 \\ 0.7 & 0.70517 & 0.00166 \\ 0.8 & 0.74962 & 0.00198 \\ 0.9 & 0.78928 & 0.00184 \\ 1.0 & 0.82302 & 0.00188 \\ 1.5 & 0.91884 & 0.00222 \\ 2.0 & 0.95833 & 0.00245 \\ 2.5 & 0.95403 & 0.00253 \\ 3.0 & 0.91497 & 0.00280 \\ 4.0 & 0.73475 & 0.00244 \\ 5.0 & 0.40870 & 0.00183\end{array}$




\section{Table BG}

K-effective Vs Uranium Concentration

30 Gallon Steel Drums filled with UF 4 - Oil Mixture

MG type concrete $\quad 10^{-4}$ (gm water/cc of air) mist density $200 \mathrm{~cm}$ Pitch 5\% Enrichment

$\begin{array}{ccc}\begin{array}{c}\text { Uranium Concentration } \\ \text { Grams U/cc Solution }\end{array} & \begin{array}{c}\text { K-effective } \\ \text { mean value } \\ 0.61020\end{array} & \begin{array}{c}\text { 1 Sigma } \\ \text { value }\end{array} \\ 0.2 & 0.76682 & 0.00145 \\ 0.3 & 0.87757 & 0.00192 \\ 0.4 & 0.95043 & 0.00219 \\ 0.5 & 1.01258 & 0.00231 \\ 0.6 & 1.05502 & 0.00228 \\ 0.7 & 1.09646 & 0.00248 \\ 0.8 & 1.12058 & 0.00252 \\ 0.9 & 1.14245 & 0.00269 \\ 1.0 & 1.18304 & 0.00289 \\ 1.5 & 1.17662 & 0.00337 \\ 2.0 & 1.12954 & 0.00312 \\ 2.5 & 1.07337 & 0.00314 \\ 3.0 & 0.83029 & 0.00267 \\ 4.0 & 0.48024 & 0.00185 \\ 5.0 & & \end{array}$

Table VG

Keffective Vs Uranium Concentration

30 Gallon Steel Drums filled with UF 4 - Oil Mixture

MG type concrete $\quad 10^{-4}$ (gm water/cc of air) mist density $200 \mathrm{~cm}$ Pitch 2\% Enrichment

$\begin{array}{ccc}\begin{array}{c}\text { Uranium Concentration } \\ \text { Grams U/cc Solution }\end{array} & \begin{array}{c}\text { Keffective } \\ \text { mean value }\end{array} & \begin{array}{c}\text { 1 Sigma } \\ \text { value }\end{array} \\ 0.2 & 0.31135 & 0.00065 \\ 0.3 & 0.42062 & 0.00095 \\ 0.4 & 0.51399 & 0.00117 \\ 0.5 & 0.58249 & 0.00136 \\ 0.6 & 0.64423 & 0.00151 \\ 0.7 & 0.70329 & 0.00163 \\ 0.8 & 0.74821 & 0.00164 \\ 0.9 & 0.78271 & 0.00196 \\ 1.0 & 0.81724 & 0.00200 \\ 1.5 & 0.90853 & 0.00229 \\ 2.0 & 0.94338 & 0.00254 \\ 2.5 & 0.93637 & 0.00250 \\ 3.0 & 0.89630 & 0.00261 \\ 4.0 & 0.70378 & 0.00255 \\ 5.0 & 0.35277 & 0.00155\end{array}$


Table EB

K-effective Vs Uranium Concentration

30 Gallon Steel Drums filled with $\mathrm{UO}_{2} \mathrm{~F}_{2}$ - Water Mixture MG type concrete $\quad 10^{-4}$ (gm water/cc of air) mist density $50 \mathrm{~cm}$ Pitch $5 \%$ Enrichment

$\begin{array}{ccc}\begin{array}{c}\text { Uranium Concentration } \\ \text { Grams U/cc Solution }\end{array} & \begin{array}{c}\text { Keffective } \\ \text { mean value } \\ 0.75879\end{array} & \begin{array}{c}\text { 1 Sigma } \\ \text { value }\end{array} \\ 0.2 & 0.92177 & 0.00116 \\ 0.3 & 1.03216 & 0.00154 \\ 0.4 & 1.11422 & 0.00187 \\ 0.5 & 1.17557 & 0.00193 \\ 0.6 & 1.21316 & 0.00205 \\ 0.7 & 1.24740 & 0.00197 \\ 0.8 & 1.27436 & 0.00229 \\ 0.9 & 1.29242 & 0.00211 \\ 1.0 & 1.33824 & 0.00234 \\ 1.5 & 1.33754 & 0.00245 \\ 2.0 & 1.31725 & 0.00260 \\ 2.5 & 1.27729 & 0.00240 \\ 3.0 & 1.20676 & 0.00233 \\ 4.0 & 1.05633 & 0.00245\end{array}$

Table YB

Keffective Vs Uranium Concentration

30 Gallon Steel Drums filled with $\mathrm{UO}_{2} \mathrm{~F}_{2}$ - Water Mixture MG type concrete $10^{-4}$ (gm water/cc of air) mist density $50 \mathrm{~cm}$ Pitch $2 \%$ Enrichment

$\begin{array}{ccc}\begin{array}{c}\text { Uranium Concentration } \\ \text { Grams U/cc Solution }\end{array} & \begin{array}{c}\text { K-effective } \\ \text { mean value } \\ 0.40485\end{array} & \begin{array}{c}\text { 1 Sigma } \\ \text { value }\end{array} \\ 0.2 & 0.00070 \\ 0.3 & 0.63082 & 0.00093 \\ 0.4 & 0.71696 & 0.00100 \\ 0.5 & 0.78101 & 0.00129 \\ 0.6 & 0.83198 & 0.00150 \\ 0.7 & 0.87957 & 0.00150 \\ 0.8 & 0.91845 & 0.00165 \\ 0.9 & 0.95044 & 0.00156 \\ 1.0 & 1.05025 & 0.00184 \\ 1.5 & 1.09116 & 0.00200 \\ 2.0 & 1.10243 & 0.00204 \\ 2.5 & 1.08776 & 0.00221 \\ 3.0 & 1.04389 & 0.00222 \\ 4.0 & 0.90229 & 0.00233\end{array}$


Table ED

Keffective Vs Uranium Concentration

30 Gallon Steel Drums filled with $\mathrm{UO}_{2} \mathrm{~F}_{2}$ - Water Mixture MG type concrete $10^{-4}$ (gm water/cc of air) mist density $75 \mathrm{~cm}$ Pitch $5 \%$ Enrichment

Uranium Concentration
Grams U/cc Solution
0.2
0.3
0.4
0.5
0.6
0.7
0.8
0.9
1.0
1.5
2.0
2.5
3.0
4.0
4.5

$\begin{array}{cc}\begin{array}{c}\text { K-effective } \\ \text { mean value }\end{array} & \begin{array}{c}\text { 1 Sigma } \\ \text { value }\end{array} \\ 0.71378 & 0.00161 \\ 0.86213 & 0.00177 \\ 0.96890 & 0.00197 \\ 1.04704 & 0.00214 \\ 1.09737 & 0.00217 \\ 1.14296 & 0.00240 \\ 1.17832 & 0.00234 \\ 1.19724 & 0.00257 \\ 1.21433 & 0.00240 \\ 1.25684 & 0.00265 \\ 1.25232 & 0.00259 \\ 1.23128 & 0.00277 \\ 1.19010 & 0.00269 \\ 1.13494 & 0.00272 \\ 0.97681 & 0.00250\end{array}$

Table YD

Keffective Vs Uranium Concentration

30 Gallon Steel Drums filled with $\mathrm{UO}_{2} \mathrm{~F}_{2}$ - Water Mixture MG type concrete $10^{-4}$ (gm water/cc of air) mist density $75 \mathrm{~cm}$ Pitch 2\% Enrichment

$\begin{array}{ccc}\begin{array}{c}\text { Uranium Concentration } \\ \text { Grams U/cc Solution }\end{array} & \begin{array}{c}\text { K-effective } \\ \text { mean value } \\ 0.37903\end{array} & \begin{array}{c}\text { 1 Sigma } \\ \text { value }\end{array} \\ 0.2 & 0.00076 \\ 0.3 & 0.59344 & 0.00097 \\ 0.4 & 0.67108 & 0.00122 \\ 0.5 & 0.72977 & 0.00150 \\ 0.6 & 0.78220 & 0.00153 \\ 0.7 & 0.82749 & 0.00160 \\ 0.8 & 0.86111 & 0.00162 \\ 0.9 & 0.89241 & 0.00197 \\ 1.0 & 0.98316 & 0.00201 \\ 1.5 & 1.01998 & 0.00236 \\ 2.0 & 1.02477 & 0.00247 \\ 2.5 & 1.01346 & 0.00250 \\ 3.0 & 0.97526 & 0.00255 \\ 4.0 & 0.82725 & 0.00239\end{array}$


Table EE

K-effective Vs Uranium Concentration

30 Gallon Steel Drums filled with $\mathrm{UO}_{2} \mathrm{~F}_{2}$ - Water Mixture MG type concrete $10^{-4}$ (gm water/cc of air) mist density $100 \mathrm{~cm}$ Pitch 5\% Enrichment

$\begin{array}{ccc}\begin{array}{c}\text { Uranium Concentration } \\ \text { Grams U/cc Solution }\end{array} & \begin{array}{c}\text { K-effective } \\ \text { mean value }\end{array} & \begin{array}{c}\text { Sigma } \\ \text { value }\end{array} \\ 0.2 & 0.68507 & 0.00162 \\ 0.3 & 0.83384 & 0.00168 \\ 0.4 & 0.93366 & 0.00201 \\ 0.5 & 1.01132 & 0.00224 \\ 0.6 & 1.05763 & 0.00247 \\ 0.7 & 1.10156 & 0.00233 \\ 0.8 & 1.12734 & 0.00265 \\ 0.9 & 1.14995 & 0.00273 \\ 1.0 & 1.17325 & 0.00256 \\ 1.5 & 1.19681 & 0.00298 \\ 2.0 & 1.20360 & 0.00318 \\ 2.5 & 1.17769 & 0.00289 \\ 3.0 & 1.14748 & 0.00303 \\ 4.0 & 1.08255 & 0.00304 \\ 4.5 & 0.91245 & 0.00259\end{array}$

Table YE

K-effective Vs Uranium Concentration

30 Gallon Steel Drums filled with $\mathrm{UO}_{2} \Sigma_{2}$ - Water Mixture MG type concrete $\quad 10^{-4}$ (gm water/cc of air) mist density $100 \mathrm{~cm}$ Pitch 2\% Enrichment

$\begin{array}{ccc}\begin{array}{c}\text { Uranium Concentration } \\ \text { Grams U/cc Solution }\end{array} & \begin{array}{c}\text { K-effective } \\ \text { mean value } \\ 0.36554\end{array} & \begin{array}{c}\text { 1 Sigma } \\ \text { value }\end{array} \\ 0.2 & 0.00082 \\ 0.3 & 0.48134 & 0.00111 \\ 0.4 & 0.56904 & 0.00127 \\ 0.5 & 0.64861 & 0.00149 \\ 0.6 & 0.70741 & 0.00148 \\ 0.7 & 0.74974 & 0.00164 \\ 0.8 & 0.79518 & 0.00183 \\ 0.9 & 0.82530 & 0.00189 \\ 1.0 & 0.86048 & 0.00188 \\ 1.5 & 0.94230 & 0.00215 \\ 2.0 & 0.97738 & 0.00252 \\ 2.5 & 0.98622 & 0.00239 \\ 3.0 & 0.96715 & 0.00251 \\ 4.0 & 0.93035 & 0.00247 \\ 4.5 & 0.76847 & 0.00237\end{array}$


Table EF

K-effective Vs Uranium Concentration

30 Gallon Steel Drums filled with $\mathrm{UO}_{2} \mathrm{~F}_{2}$ - Water Mixture MG type concrete $\quad 10^{-4}$ (gm water/cc of air) mist density $150 \mathrm{~cm}$ Pitch $5 \%$ Enrichment

$\begin{array}{ccc}\begin{array}{c}\text { Uranium Concentration } \\ \text { Grams U/cc Solution }\end{array} & \begin{array}{c}\text { K-effective } \\ \text { mean value } \\ 0.66445\end{array} & \begin{array}{c}\text { 1 Sigma } \\ \text { value } \\ 0.2\end{array} \\ 0.3 & 0.80594 & 0.00155 \\ 0.4 & 0.90326 & 0.00233 \\ 0.5 & 0.96951 & 0.00241 \\ 0.6 & 1.02157 & 0.00261 \\ 0.7 & 1.05983 & 0.00280 \\ 0.8 & 1.08514 & 0.00194 \\ 0.9 & 1.10727 & 0.00242 \\ 1.0 & 1.12031 & 0.00284 \\ 1.5 & 1.15975 & 0.00295 \\ 2.0 & 1.15786 & 0.00305 \\ 2.5 & 1.12476 & 0.00292 \\ 3.0 & 1.08571 & 0.00297 \\ 4.0 & 1.02511 & 0.00284 \\ 4.5 & 0.83552 & 0.00265\end{array}$

Table YF

Keffective Vs Uranium Concentration

30 Gallon Steel Drums filled with $\mathrm{UO}_{2} \mathrm{~F}_{2}$ - Water Mixture MG type concrete $\quad 10^{-4}$ (gm water/cc of air) mist density $150 \mathrm{~cm}$ Pitch 2\% Enrichment

$\begin{array}{ccc}\begin{array}{c}\text { Uranium Concentration } \\ \text { Grams U/cc Solution }\end{array} & \begin{array}{c}\text { K-effective } \\ \text { mean value } \\ 0.2\end{array} & \begin{array}{c}\text { 1 Sigma } \\ \text { value }\end{array} \\ 0.3 & 0.35096 & 0.00088 \\ 0.4 & 0.46498 & 0.00121 \\ 0.5 & 0.55446 & 0.00140 \\ 0.6 & 0.62489 & 0.00159 \\ 0.7 & 0.67991 & 0.00180 \\ 0.8 & 0.72437 & 0.00194 \\ 0.9 & 0.76234 & 0.00181 \\ 1.0 & 0.79632 & 0.00188 \\ 1.5 & 0.82765 & 0.00198 \\ 2.0 & 0.90973 & 0.00228 \\ 2.5 & 0.92819 & 0.00252 \\ 3.0 & 0.93328 & 0.00258 \\ 4.0 & 0.91655 & 0.00268 \\ 4.5 & 0.88167 & 0.00262 \\ & 0.70079 & 0.00256\end{array}$


Table EG

K-effective Vs Uranium Concentration

30 Gallon Steel Drums filled with $\mathrm{UO}_{2} \mathrm{~F}_{2}$ - Water Mixture MG type concrete $10^{-4}$ (gm water/cc of air) mist density $200 \mathrm{~cm}$ Pitch 5\% Enrichment

\section{Uranium Concentration Grams U/ce Solution}

$$
0.2
$$

0.3

0.4

0.5

0.6

0.7

0.8

0.9

1.0

1.5

2.0

2.5

3.0

4.0

4.5

K-effective
mean value
0.65356
0.79378
0.89267
0.95946
0.99961
1.04452
1.07314
1.09672
1.10717
1.13105
1.12097
1.09107
1.05719
0.99436
0.79874

1 Sigma value 0.00177 0.00210 0.00247 0.00240 0.00278 0.00269 0.00275 0.00289 0.00267 0.00290 0.00319 0.00298 0.00319 0.00304 0.00295

Table YG

K-ffective Vs Uranium Concentration

30 Gallon Steel Drums filled with $\mathrm{UO}_{2} \mathrm{~F}_{2}$ - Water Mixture MG type concrete $10^{-4}$ (gm water/cc of air) mist density $200 \mathrm{~cm}$ Pitch 2\% Enrichment

$\begin{array}{ccc}\begin{array}{c}\text { Uranium Concentration } \\ \text { Grams U/cc Solution }\end{array} & \begin{array}{c}\text { Keffective } \\ \text { mean value } \\ 0.34859\end{array} & \begin{array}{c}\text { 1 Sigma } \\ \text { value } \\ 0.2\end{array} \\ 0.3 & 0.45677 & 0.00092 \\ 0.4 & 0.54137 & 0.00115 \\ 0.5 & 0.61449 & 0.00141 \\ 0.6 & 0.66878 & 0.00163 \\ 0.7 & 0.71459 & 0.00191 \\ 0.8 & 0.75382 & 0.00207 \\ 0.9 & 0.78257 & 0.00193 \\ 1.0 & 0.80816 & 0.00232 \\ 1.5 & 0.88633 & 0.00242 \\ 2.0 & 0.91930 & 0.00173 \\ 2.5 & 0.91588 & 0.00257 \\ 3.0 & 0.89129 & 0.00264 \\ 4.0 & 0.85997 & 0.00262 \\ 4.5 & 0.66918 & 0.00255\end{array}$


Table CC

K-effective Vs Uranium Concentration

55 Gallon Steel Drums filled with $\mathrm{UF}_{4}$ - Oil Mixture

MG type concrete $\quad 10^{-4}$ (gm water/cc of air) mist density $60 \mathrm{~cm}$ Pitch 5\% Enrichment

$\begin{array}{ccc}\begin{array}{c}\text { Uranium Concentration } \\ \text { Grams U/cc Solution }\end{array} & \begin{array}{c}\text { Keffective } \\ \text { mean value }\end{array} & \begin{array}{c}\text { 1 Sigma } \\ \text { value }\end{array} \\ 0.2 & 0.68484 & 0.00102 \\ 0.3 & 0.85981 & 0.00125 \\ 0.4 & 0.98805 & 0.00144 \\ 0.5 & 1.07531 & 0.00168 \\ 0.6 & 1.14604 & 0.00180 \\ 0.7 & 1.19864 & 0.00197 \\ 0.8 & 1.24349 & 0.00199 \\ 0.9 & 1.27510 & 0.00197 \\ 1.0 & 1.29636 & 0.00227 \\ 1.5 & 1.36573 & 0.00210 \\ 2.0 & 1.37693 & 0.00225 \\ 2.5 & 1.34957 & 0.00243 \\ 3.0 & 1.29921 & 0.00253 \\ 4.0 & 1.11876 & 0.00238 \\ 5.0 & 0.75191 & 0.00176\end{array}$

Table WC

K-effective Vs Uranium Concentration

55 Gallon Steel Drums filled with UF 4 - Oil Mixture

MG type concrete $10^{-4}$ (gm water/cc of air) mist density $60 \mathrm{~cm}$ Pitch $2 \%$ Enrichment

$\begin{array}{ccc}\begin{array}{c}\text { Uranium Concentration } \\ \text { Grams U/cc Solution }\end{array} & \begin{array}{c}\text { Keffective } \\ \text { mean value }\end{array} & \begin{array}{c}\text { 1 Sigma } \\ \text { value }\end{array} \\ 0.2 & 0.35015 & 0.00052 \\ 0.3 & 0.47432 & 0.00078 \\ 0.4 & 0.57921 & 0.00088 \\ 0.5 & 0.66202 & 0.00102 \\ 0.6 & 0.73480 & 0.00111 \\ 0.7 & 0.79363 & 0.00129 \\ 0.8 & 0.84776 & 0.00123 \\ 0.9 & 0.89108 & 0.00142 \\ 1.0 & 0.93292 & 0.00156 \\ 1.5 & 1.05904 & 0.00180 \\ 2.0 & 1.11699 & 0.00184 \\ 2.5 & 1.12725 & 0.00195 \\ 3.0 & 1.10977 & 0.00198 \\ 4.0 & 0.96444 & 0.00224 \\ 5.0 & 0.54060 & 0.00162\end{array}$


Table CD

K-effective Vs Uranium Concentration

55 Gallon Steel Drums filled with UF 4 - Oil Mixture

MG type concrete $\quad 10^{-4}$ (gm water/cc of air) mist density $75 \mathrm{~cm}$ Pitch 5\% Enrichment

$\begin{array}{ccc}\begin{array}{c}\text { Uranium Concentration } \\ \text { Grams U/cc Solution }\end{array} & \begin{array}{c}\text { Keffective } \\ \text { mean value } \\ 0.67098\end{array} & \begin{array}{c}\text { Sigma } \\ \text { value }\end{array} \\ 0.2 & 0.84692 & 0.00110 \\ 0.3 & 0.96951 & 0.00157 \\ 0.4 & 1.05438 & 0.00167 \\ 0.5 & 1.12093 & 0.00200 \\ 0.6 & 1.17589 & 0.00201 \\ 0.7 & 1.21283 & 0.00221 \\ 0.8 & 1.24302 & 0.00203 \\ 0.9 & 1.27186 & 0.00199 \\ 1.0 & 1.34231 & 0.00257 \\ 1.5 & 1.33808 & 0.00256 \\ 2.0 & 1.31639 & 0.00268 \\ 2.5 & 1.26019 & 0.00264 \\ 3.0 & 1.07289 & 0.00226 \\ 4.0 & 0.73883 & 0.00192\end{array}$

Table WD

K-effective Vs Uranium Concentration

55 Gallon Steel Drums filled with UF 4 - Oil Mixture

MG type concrete $10^{-4}$ (gm water/cc of air) mist density

$75 \mathrm{~cm}$ Pitch $2 \%$ Enrichment

$\begin{array}{ccc}\begin{array}{c}\text { Uranium Concentration } \\ \text { Grams U/cc Solution }\end{array} & \begin{array}{c}\text { Keffective } \\ \text { mean value } \\ 0.34422\end{array} & \begin{array}{c}\text { 1 Sigma } \\ \text { value }\end{array} \\ 0.2 & 0.00060 \\ 0.3 & 0.46597 & 0.00081 \\ 0.4 & 0.56979 & 0.00091 \\ 0.5 & 0.65068 & 0.00112 \\ 0.6 & 0.71970 & 0.00120 \\ 0.7 & 0.77848 & 0.00130 \\ 0.8 & 0.82871 & 0.00149 \\ 0.9 & 0.87188 & 0.00148 \\ 1.0 & 0.90967 & 0.00158 \\ 1.5 & 1.03148 & 0.00175 \\ 2.0 & 1.08898 & 0.00214 \\ 2.5 & 1.10104 & 0.00205 \\ 3.0 & 1.07562 & 0.00217 \\ 4.0 & 0.92833 & 0.00249 \\ 5.0 & 0.54548 & 0.00176\end{array}$


Table CE

K-effective Vs Uranium Concentration

55 Gallon Steel Drums filled with UF 4 - Oil Mixture

MG type concrete $\quad 10^{-4}$ (gm water/cc of air) mist density $100 \mathrm{~cm}$ Pitch 5\% Enrichment

$\begin{array}{ccc}\begin{array}{c}\text { Uranium Concentration } \\ \text { Grams U/cc Solution }\end{array} & \begin{array}{c}\text { K-effective } \\ \text { mean value }\end{array} & \begin{array}{c}\text { 1 Sigma } \\ \text { value }\end{array} \\ 0.2 & 0.66202 & 0.00120 \\ 0.3 & 0.83120 & 0.00164 \\ 0.4 & 0.95024 & 0.00161 \\ 0.5 & 1.03636 & 0.00185 \\ 0.6 & 1.09462 & 0.00196 \\ 0.7 & 1.14984 & 0.00212 \\ 0.8 & 1.19600 & 0.00225 \\ 0.9 & 1.21610 & 0.00234 \\ 1.0 & 1.24684 & 0.00230 \\ 1.5 & 1.30444 & 0.00253 \\ 2.0 & 1.30150 & 0.00276 \\ 2.5 & 1.27543 & 0.00277 \\ 3.0 & 1.21900 & 0.00269 \\ 4.0 & 1.03426 & 0.00238 \\ 5.0 & 0.70110 & 0.00194\end{array}$

Table WE

K-effective Vs Uranium Concentration

55 Gallon Steel Drums filled with $\mathrm{UF}_{4}$ - Oil Mixture

MG type concrete $10^{-4}$ (gm water/cc of air) mist density $100 \mathrm{~cm}$ Pitch 2\% Enrichment

$\begin{array}{ccc}\begin{array}{c}\text { Uranium Concentration } \\ \text { Grams U/cc Solution }\end{array} & \begin{array}{c}\text { K-effective } \\ \text { mean value } \\ 0.33794\end{array} & \begin{array}{c}\text { 1 Sigma } \\ \text { value }\end{array} \\ 0.2 & 0.00062 \\ 0.3 & 0.45587 & 0.00081 \\ 0.4 & 0.55785 & 0.00112 \\ 0.5 & 0.63661 & 0.00117 \\ 0.6 & 0.70522 & 0.00126 \\ 0.7 & 0.76674 & 0.00141 \\ 0.8 & 0.81572 & 0.00152 \\ 0.9 & 0.85540 & 0.00172 \\ 1.0 & 0.88660 & 0.00167 \\ 1.5 & 1.00719 & 0.00189 \\ 2.0 & 1.05196 & 0.00235 \\ 2.5 & 1.06088 & 0.00231 \\ 3.0 & 1.03537 & 0.00253 \\ 4.0 & 0.87851 & 0.00227 \\ 5.0 & 0.52266 & 0.00191\end{array}$


Table CF

K-effective Vs Uranium Concentration

55 Gallon Steel Drums filled with $\mathrm{UF}_{4}$ - Oil Mixture

MG type concrete $\quad 10^{-4}$ (gm water/cc of air) mist density $150 \mathrm{~cm}$ Pitch 5\% Enrichment

$\begin{array}{ccc}\begin{array}{c}\text { Uranium Concentration } \\ \text { Grams U/cc Solution }\end{array} & \begin{array}{c}\text { K-effective } \\ \text { mean value } \\ 0.64993\end{array} & \begin{array}{c}\text { 1 Sigma } \\ \text { value }\end{array} \\ 0.2 & 0.01349 & 0.00169 \\ 0.3 & 0.92866 & 0.00181 \\ 0.4 & 1.01745 & 0.00204 \\ 0.5 & 1.08023 & 0.00212 \\ 0.6 & 1.12416 & 0.00237 \\ 0.7 & 1.16524 & 0.00267 \\ 0.8 & 1.18843 & 0.00229 \\ 0.9 & 1.21676 & 0.00264 \\ 1.0 & 1.27073 & 0.00278 \\ 1.5 & 1.26418 & 0.00284 \\ 2.0 & 1.22869 & 0.00272 \\ 2.5 & 1.16669 & 0.00311 \\ 3.0 & 0.96570 & 0.00270 \\ 4.0 & 0.61537 & 0.00214\end{array}$

Table WF

K-effective Vs Uranium Concentration

55 Gallon Steel Drums filled with $\mathrm{UF}_{4}$ - Oil Mixture

MG type concrete $10^{-4}$ (gm water/cc of air) mist density $150 \mathrm{~cm}$ Pitch 2\% Enrichment

$\begin{array}{ccc}\begin{array}{c}\text { Uranium Concentration } \\ \text { Grams U/cc Solution }\end{array} & \begin{array}{c}\text { K-effective } \\ \text { mean value } \\ 0.33129\end{array} & \begin{array}{c}\text { 1 Sigma } \\ \text { value }\end{array} \\ 0.2 & 0.04956 & 0.0067 \\ 0.3 & 0.54802 & 0.00109 \\ 0.4 & 0.62265 & 0.00117 \\ 0.5 & 0.69022 & 0.00132 \\ 0.6 & 0.74868 & 0.00148 \\ 0.7 & 0.79096 & 0.00149 \\ 0.8 & 0.83735 & 0.00178 \\ 0.9 & 0.87099 & 0.00163 \\ 1.0 & 0.98180 & 0.00222 \\ 1.5 & 1.02619 & 0.00241 \\ 2.0 & 1.02514 & 0.00238 \\ 2.5 & 0.99101 & 0.00225 \\ 3.0 & 0.82525 & 0.00242 \\ 4.0 & 0.46033 & 0.00167\end{array}$




\section{Table CG}

K-effective Vs Uranium Concentration

55 Gallon Steel Drums filled with UF 4 - Oil Mixture MG type concrete $\quad 10^{-4}$ (gm water/cc of air) mist density $200 \mathrm{~cm}$ Pitch 5\% Enrichment

$\begin{array}{ccc}\begin{array}{c}\text { Uranium Concentration } \\ \text { Grams U/cc Solution }\end{array} & \begin{array}{c}\text { Keffective } \\ \text { mean value } \\ 0.64345\end{array} & \begin{array}{c}\text { 1 Sigma } \\ \text { value }\end{array} \\ 0.2 & 0.00139 \\ 0.3 & 0.80771 & 0.00170 \\ 0.4 & 0.92328 & 0.00203 \\ 0.5 & 1.00968 & 0.00215 \\ 0.6 & 1.06293 & 0.00211 \\ 0.7 & 1.11795 & 0.00231 \\ 0.8 & 1.15954 & 0.00226 \\ 0.9 & 1.18185 & 0.00237 \\ 1.0 & 1.20931 & 0.00238 \\ 1.5 & 1.25416 & 0.00270 \\ 2.0 & 1.25383 & 0.00295 \\ 2.5 & 1.20829 & 0.00281 \\ 3.0 & 1.15172 & 0.00303 \\ 4.0 & 0.93712 & 0.00306 \\ 5.0 & 0.55986 & 0.00213\end{array}$

Table WG

Keffective $V_{\text {a }}$ Uranium Concentration

55 Gallon Steel Druns filled with $\mathrm{UF}_{4}$ - Oil Mixture

MG type concrete $10^{-4}$ (gm water/cc of air) mist density $200 \mathrm{~cm}$ Pitch 2\% Enrichment

$\begin{array}{ccc}\begin{array}{c}\text { Uranium Concentration } \\ \text { Grams U/cc Solution }\end{array} & \begin{array}{c}\text { K-effective } \\ \text { mean value } \\ 0.33008\end{array} & \begin{array}{c}\text { 1 Sigma } \\ \text { value }\end{array} \\ 0.2 & 0.44649 & 0.00066 \\ 0.3 & 0.54214 & 0.00088 \\ 0.4 & 0.61683 & 0.00129 \\ 0.5 & 0.68536 & 0.00139 \\ 0.6 & 0.74302 & 0.00159 \\ 0.7 & 0.78741 & 0.00180 \\ 0.8 & 0.83240 & 0.00181 \\ 0.9 & 0.86807 & 0.00179 \\ 1.0 & 0.97277 & 0.00214 \\ 1.5 & 1.01532 & 0.00249 \\ 2.0 & 1.00764 & 0.00265 \\ 2.5 & 0.97171 & 0.00275 \\ 3.0 & 0.79726 & 0.00258 \\ 4.0 & 0.40642 & 0.00168 \\ 5.0 & & \end{array}$




\section{Table CH}

Keffective Vs Uranium Concentration

55 Gallon Steel Drums filled with UF 4 - Oil Mixture

MG type concrete $\quad 10^{-4}$ (gm water/cc of air) mist density $300 \mathrm{~cm}$ Pitch 5\% Enrichment

$\begin{array}{ccc}\begin{array}{c}\text { Uranium Concentration } \\ \text { Grams U/cc Solution }\end{array} & \begin{array}{c}\text { Keffective } \\ \text { mean value }\end{array} & \begin{array}{c}\text { 1 Sigma } \\ \text { value }\end{array} \\ 0.2 & 0.64059 & 0.00122 \\ 0.3 & 0.80682 & 0.00164 \\ 0.4 & 0.91756 & 0.00202 \\ 0.5 & 0.99652 & 0.00214 \\ 0.6 & 1.06067 & 0.00233 \\ 0.7 & 1.10925 & 0.00246 \\ 0.8 & 1.14551 & 0.00248 \\ 0.9 & 1.18001 & 0.00265 \\ 1.0 & 1.19825 & 0.00264 \\ 1.5 & 1.24271 & 0.00278 \\ 2.0 & 1.23979 & 0.00303 \\ 2.5 & 1.19369 & 0.00295 \\ 3.0 & 1.13124 & 0.00313 \\ 4.0 & 0.91115 & 0.00280 \\ 5.0 & 0.50260 & 0.00175\end{array}$

Table WH

K-effective Vs Uranium $\mathrm{C}$, tration 55 Gallon Steel Drums filled wi Oil Mixture

MG type concrete $\quad 10^{-4}$ (gm wate ir) mist density $300 \mathrm{~cm}$ Pitch $2 \%$ Enx. went

$\begin{array}{ccc}\begin{array}{c}\text { Uranium Concentration } \\ \text { Grams U/cc Solution }\end{array} & \begin{array}{c}\text { Keffective } \\ \text { mean value }\end{array} & \begin{array}{c}\text { 1 Sigma } \\ \text { value }\end{array} \\ 0.2 & 0.32814 & 0.00073 \\ 0.3 & 0.44496 & 0.00086 \\ 0.4 & 0.53941 & 0.00110 \\ 0.5 & 0.61817 & 0.00130 \\ 0.6 & 0.68197 & 0.00150 \\ 0.7 & 0.74167 & 0.00155 \\ 0.8 & 0.78662 & 0.00166 \\ 0.9 & 0.82280 & 0.00183 \\ 1.0 & 0.85697 & 0.00195 \\ 1.5 & 0.95737 & 0.00233 \\ 2.0 & 1.00131 & 0.00256 \\ 2.5 & 1.00057 & 0.00253 \\ 3.0 & 0.96109 & 0.00262 \\ 4.0 & 0.77237 & 0.00262 \\ 5.0 & 0.35451 & 0.00150\end{array}$




\section{Table CI}

Keffective Vs Uranium Concentration

55 Gallon Steel Drums filled with $\mathrm{UF}_{4}$ - Oil Mixture

MG type concrete $\quad 10^{-4}$ (gm water/cc of air) mist density $400 \mathrm{~cm}$ Pitch 5\% Enrichment

$\begin{array}{ccc}\begin{array}{c}\text { Uranium Concentration } \\ \text { Grams U/cc Solution }\end{array} & \begin{array}{c}\text { K-effective } \\ \text { mean value } \\ 0.63828\end{array} & \begin{array}{c}1 \text { Sigma } \\ \text { value }\end{array} \\ 0.2 & 0.80531 & 0.00134 \\ 0.3 & 0.91973 & 0.00160 \\ 0.4 & 1.00023 & 0.00206 \\ 0.5 & 1.06009 & 0.00220 \\ 0.6 & 1.10364 & 0.00251 \\ 0.7 & 1.15175 & 0.00246 \\ 0.8 & 1.16832 & 0.00254 \\ 0.9 & 1.20064 & 0.00249 \\ 1.0 & 1.23975 & 0.00269 \\ 1.5 & 1.23297 & 0.00302 \\ 2.0 & 1.18824 & 0.00294 \\ 2.5 & 1.12439 & 0.00306 \\ 3.0 & 0.89398 & 0.00282 \\ 4.0 & 0.48295 & 0.00175\end{array}$

\section{Table WI}

Keffective Vs Uranium Concentration

55 Gallon Steel Drums filled with UF 4 - Oil Mixture

MG type concrete $\quad 10^{-4}$ (gm water/cc of air) mist density $400 \mathrm{~cm}$ Pitch 2\% Enrichment

$\begin{array}{ccc}\begin{array}{c}\text { Uranium Concentration } \\ \text { Grams U/cc Solution }\end{array} & \begin{array}{c}\text { Keffective } \\ \text { mean value }\end{array} & \begin{array}{c}\text { Sigma } \\ \text { value }\end{array} \\ 0.2 & 0.32604 & 0.00070 \\ 0.3 & 0.44354 & 0.00094 \\ 0.4 & 0.53833 & 0.00108 \\ 0.5 & 0.61338 & 0.00141 \\ 0.6 & 0.68401 & 0.00149 \\ 0.7 & 0.73372 & 0.00165 \\ 0.8 & 0.78574 & 0.00179 \\ 0.9 & 0.81870 & 0.00184 \\ 1.0 & 0.85958 & 0.00191 \\ 1.5 & 0.96370 & 0.00212 \\ 2.0 & 0.99617 & 0.00222 \\ 2.5 & 0.99111 & 0.00266 \\ 3.0 & 0.95980 & 0.00278 \\ 4.0 & 0.76144 & 0.00248 \\ 5.0 & 0.33358 & 0.00140\end{array}$




\section{Table CJ}

K-effective Vs Uranium Concentration

55 Gallon Steel Drums filled with UF 4 - Oil Mixture

MG type concrete $\quad 10^{-4}$ (gm water/cc of air) mist density

$500 \mathrm{~cm}$ Pitch 5\% Enrichment

$\begin{array}{ccc}\begin{array}{c}\text { Uranium Concentration } \\ \text { Grams U/cc Solution }\end{array} & \begin{array}{c}\text { Keffective } \\ \text { mean value }\end{array} & \begin{array}{c}\text { 1 Sigma } \\ \text { value }\end{array} \\ 0.2 & 0.63983 & 0.00129 \\ 0.3 & 0.79969 & 0.00186 \\ 0.4 & 0.91700 & 0.00211 \\ 0.5 & 0.99519 & 0.00216 \\ 0.6 & 1.06723 & 0.00234 \\ 0.7 & 1.10859 & 0.00227 \\ 0.8 & 1.14189 & 0.00263 \\ 0.9 & 1.17207 & 0.00250 \\ 1.0 & 1.19074 & 0.00301 \\ 1.5 & 1.23841 & 0.00292 \\ 2.0 & 1.23357 & 0.00341 \\ 2.5 & 1.18082 & 0.00309 \\ 3.0 & 1.12689 & 0.00327 \\ 4.0 & 0.89404 & 0.00294 \\ 5.0 & 0.47478 & 0.00154\end{array}$

Table WJ

Keffective Vs Uranium Concentration

55 Gallon Steel Drums filled with $\mathrm{UF}_{4}$ - Oil Mixture

MG type concrete $\quad 10^{-4}$ (gm water/cc of air) mist density

$500 \mathrm{~cm}$ Pitch 2\% Enrichment

$\begin{array}{ccc}\begin{array}{c}\text { Uranium Concentration } \\ \text { Grams U/cc Solution }\end{array} & \begin{array}{c}\text { Keffective } \\ \text { mean value }\end{array} & \begin{array}{c}\text { 1 Sigma } \\ \text { value }\end{array} \\ 0.2 & 0.32786 & 0.00067 \\ 0.3 & 0.44225 & 0.00091 \\ 0.4 & 0.53889 & 0.00114 \\ 0.5 & 0.61383 & 0.00151 \\ 0.6 & 0.68081 & 0.00157 \\ 0.7 & 0.74083 & 0.00164 \\ 0.8 & 0.78034 & 0.00174 \\ 0.9 & 0.82321 & 0.00177 \\ 1.0 & 0.85905 & 0.00196 \\ 1.5 & 0.96031 & 0.00223 \\ 2.0 & 0.99418 & 0.00260 \\ 2.5 & 0.98958 & 0.00251 \\ 3.0 & 0.94943 & 0.00299 \\ 4.0 & 0.76408 & 0.00275 \\ 5.0 & 0.32505 & 0.00140\end{array}$




\section{Table FC}

K-effective Vs Uranium Concentration

55 Gallon Steel Drums filled with $\mathrm{UO}_{2} \mathrm{~F}_{2}$ - Water Mixture MG type concrete $\quad 10^{-4}$ (gm water/cc of air) mist density $60 \mathrm{~cm}$ Pitch 5\% Enrichment

$\begin{array}{ccc}\begin{array}{c}\text { Uranium Concentration } \\ \text { Grams U/cc Solution }\end{array} & \begin{array}{c}\text { K-effective } \\ \text { mean value } \\ 0.77923\end{array} & \begin{array}{c}\text { 1 Sigma } \\ \text { value }\end{array} \\ 0.2 & 0.00191 \\ 0.3 & 0.94228 & 0.00191 \\ 0.4 & 1.05668 & 0.00256 \\ 0.5 & 1.13394 & 0.00299 \\ 1.0 & 1.31671 & 0.00311 \\ 1.5 & 1.36719 & 0.00333 \\ 2.0 & 1.35639 & 0.00323 \\ 2.5 & 1.33872 & 0.00287 \\ 3.0 & 1.30616 & 0.00369 \\ 4.0 & 1.23043 & 0.00325 \\ 4.5 & 1.07048 & 0.00345 \\ 5.0 & 0.73663 & 0.00263\end{array}$

Table ZC

Keffective Vs Uranium Concentration

55 Gallon Steel Drums filled with $\mathrm{UO}_{2} \mathrm{~F}_{2}$ - Water Mixture MG type concrete $\quad 10^{-4}$ (gm water/cc of air) mist density $60 \mathrm{~cm}$ Pitch 2\% Enrichment

$\begin{array}{ccc}\begin{array}{c}\text { Uranium Concentration } \\ \text { Grams U/cc Solution }\end{array} & \begin{array}{c}\text { K-effective } \\ \text { mean value }\end{array} & \begin{array}{c}\text { 1 Sigma } \\ \text { value }\end{array} \\ 0.2 & 0.41446 & 0.00095 \\ 0.3 & 0.54587 & 0.00131 \\ 0.4 & 0.64942 & 0.00152 \\ 0.5 & 0.73315 & 0.00178 \\ 1.0 & 0.97385 & 0.00238 \\ 1.5 & 1.07411 & 0.00259 \\ 2.0 & 1.11424 & 0.00256 \\ 2.5 & 1.12035 & 0.00281 \\ 3.0 & 1.10415 & 0.00310 \\ 4.0 & 1.06583 & 0.00304 \\ 4.5 & 0.92393 & 0.00320 \\ 5.0 & 0.53282 & 0.00306\end{array}$


Table FD

Keffective Vs Uranium Concentration

55 Gallon Steel Drums filled with $\mathrm{UO}_{2} \mathrm{~F}_{2}$ - Water Mixture MG type concrete $10^{-4}$ (gm water/cc of air) mist density $75 \mathrm{~cm}$ Pitch $5 \%$ Enrichment

$\begin{array}{ccc}\begin{array}{c}\text { Uranium Concentration } \\ \text { Grams U/cc Solution }\end{array} & \begin{array}{c}\text { K-effective } \\ \text { mean value }\end{array} & \begin{array}{c}\text { Sigma } \\ \text { value }\end{array} \\ 0.2 & 0.75670 & 0.00196 \\ 0.3 & 0.92138 & 0.00239 \\ 0.4 & 1.02247 & 0.00274 \\ 0.5 & 1.11116 & 0.00261 \\ 1.0 & 1.27561 & 0.00338 \\ 1.5 & 1.32603 & 0.00351 \\ 2.0 & 1.31761 & 0.00349 \\ 2.5 & 1.29872 & 0.00376 \\ 3.0 & 1.26519 & 0.00394 \\ 4.0 & 1.19893 & 0.00340 \\ 4.5 & 1.03816 & 0.00383 \\ 5.0 & 0.72735 & 0.00282\end{array}$

Table ZD

Keffective Vs Uranium Concentration

55 Gallon Steel Drums filled with $\mathrm{UO}_{2} \mathrm{~F}_{2}$ - Water Mixture MG type concrete $10^{-4}$ (gm water/cc of air) mist density $75 \mathrm{~cm}$ Pitch $2 \%$ Enrichment

$\begin{array}{ccc}\begin{array}{c}\text { Uranium Concentration } \\ \text { Grams U/cc Solution }\end{array} & \begin{array}{c}\text { Keffective } \\ \text { mean value }\end{array} & \begin{array}{c}\text { 1 Sigma } \\ \text { value }\end{array} \\ 0.2 & 0.40029 & 0.00092 \\ 0.3 & 0.52959 & 0.00145 \\ 0.4 & 0.63258 & 0.00160 \\ 0.5 & 0.71770 & 0.00203 \\ 1.0 & 0.94737 & 0.00266 \\ 1.5 & 1.04523 & 0.00318 \\ 2.0 & 1.07348 & 0.00285 \\ 2.5 & 1.08393 & 0.00285 \\ 3.0 & 1.07298 & 0.00319 \\ 4.0 & 1.02818 & 0.00334 \\ 4.5 & 0.88312 & 0.00305 \\ 5.0 & 0.53744 & 0.00230\end{array}$




\section{Table FE}

K-effective Vs Uranium Concentration

55 Gallon Steel Drums filled with $\mathrm{UO}_{2} \mathrm{~F}_{2}$ - Water Mixture MG type concrete $\quad 10^{-4}$ (gm water/cc of air) mist density $100 \mathrm{~cm}$ Pitch 5\% Enrichment

Uranium Concentration
Grams U/cc Solution
0.2
0.3
0.4
0.5
1.0
1.5
2.0
2.5
3.0
4.0
4.5
5.0

K-effective
mean value
0.73197
0.89164
0.99870
1.07837
1.24479
1.28088
1.28279
1.26204
1.21552
1.15911
0.99409
0.68340

1 Sigma

value

0.00211

0.00237

0.00290

0.00293

0.00329

0.00360

0.00377

$\mathbf{0 . 0 0 3 8 4}$

$\mathbf{0 . 0 0 3 8 4}$

0.00370

0.00363

0.00304

Table ZE

Keffective Vs Uranium Concentration

55 Gallon Steel Drums filled with $\mathrm{UO}_{2} \mathrm{~F}_{2}$ - Water Mixture MG type concrete $\quad 10^{-4}$ (gm water/cc of air) mist density $100 \mathrm{~cm}$ Pitch 2\% Enrichment

$\begin{array}{ccc}\begin{array}{c}\text { Uranium Concentration } \\ \text { Grams U/cc Solution }\end{array} & \begin{array}{c}\text { Keffective } \\ \text { mean value }\end{array} & \begin{array}{c}\text { 1 Sigma } \\ \text { value }\end{array} \\ 0.2 & 0.39027 & 0.00120 \\ 0.3 & 0.51559 & 0.00134 \\ 0.4 & 0.61544 & 0.00179 \\ 0.5 & 0.69106 & 0.00194 \\ 1.0 & 0.91813 & 0.00257 \\ 1.5 & 1.01342 & 0.00250 \\ 2.0 & 1.04360 & 0.00315 \\ 2.5 & 1.04991 & 0.00333 \\ 3.0 & 1.04087 & 0.00302 \\ 4.0 & 0.99815 & 0.00328 \\ 4.5 & 0.84454 & 0.00354 \\ 5.0 & 0.52822 & 0.00277\end{array}$


Table FF

K-effective Vs Uranium Concentration

55 Gallon Steel Drums filled with $\mathrm{UO}_{2} \mathrm{~F}_{2}$ - Water Mixture MG type concrete $\quad 10^{-4}$ (gm water/cc of air) mist density $150 \mathrm{~cm}$ Pitch 5\% Enrichment

$\begin{array}{ccc}\begin{array}{c}\text { Uranium Concentration } \\ \text { Grams U/cc Solution }\end{array} & \begin{array}{c}\text { K-effective } \\ \text { mean value }\end{array} & \begin{array}{c}\text { 1 Sigma } \\ \text { value }\end{array} \\ 0.2 & 0.71350 & 0.00245 \\ 0.3 & 0.86426 & 0.00263 \\ 0.4 & 0.96549 & 0.00292 \\ 0.5 & 1.04446 & 0.00305 \\ 1.0 & 1.20727 & 0.00419 \\ 1.5 & 1.23902 & 0.00381 \\ 2.0 & 1.22885 & 0.00366 \\ 2.5 & 1.20685 & 0.00395 \\ 3.0 & 1.16948 & 0.00439 \\ 4.0 & 1.11273 & 0.00335 \\ 4.5 & 0.93230 & 0.00378 \\ 5.0 & 0.59892 & 0.00309\end{array}$

Table ZF

K-effective Vs Uranium Concentration

55 Gallon Steel Drums filled with $\mathrm{UO}_{2} \mathrm{~F}_{2}$ - Water Mixture MG type concrete $\quad 10^{-4}$ (gm water/cc of air) mist density $150 \mathrm{~cm}$ Pitch 2\% Enrichment

$\begin{array}{ccc}\begin{array}{c}\text { Uranium Concentration } \\ \text { Grams U/cc Solution }\end{array} & \begin{array}{c}\text { Keffective } \\ \text { mean value }\end{array} & \begin{array}{c}\text { 1 Sigma } \\ \text { value }\end{array} \\ 0.2 & 0.38045 & 0.00138 \\ 0.3 & 0.49979 & 0.00150 \\ 0.4 & 0.59608 & 0.00199 \\ 0.5 & 0.66616 & 0.00227 \\ 1.0 & 0.88432 & 0.00279 \\ 1.5 & 0.97615 & 0.00314 \\ 2.0 & 1.00487 & 0.00290 \\ 2.5 & 1.01340 & 0.00345 \\ 3.0 & 0.99881 & 0.00338 \\ 4.0 & 0.96070 & 0.00325 \\ 4.5 & 0.79089 & 0.00337 \\ 5.0 & 0.45893 & 0.00302\end{array}$


Table FG

K-effective Vs Uranium Concentration

55 Gallon Steel Drums filled with $\mathrm{UO}_{2} \mathrm{~F}_{2}$ - Water Mixture MG type concrete $10^{-4}$ (gm water/cc of air) mist density $200 \mathrm{~cm}$ Pitch 5\% Enrichment

$\begin{array}{ccc}\begin{array}{c}\text { Uranium Concentration } \\ \text { Grams U/cc Solution }\end{array} & \begin{array}{c}\text { K-effective } \\ \text { mean value }\end{array} & \begin{array}{c}\text { 1 Sigma } \\ \text { value }\end{array} \\ 0.2 & 0.70160 & 0.00253 \\ 0.3 & 0.85394 & 0.00262 \\ 0.4 & 0.95393 & 0.00292 \\ 0.5 & 1.03221 & 0.00318 \\ 1.0 & 1.18252 & 0.00391 \\ 1.5 & 1.22285 & 0.00432 \\ 2.0 & 1.22309 & 0.00440 \\ 2.5 & 1.19263 & 0.00370 \\ 3.0 & 1.14166 & 0.00446 \\ 4.0 & 1.09038 & 0.00400 \\ 4.5 & 0.89347 & 0.00371 \\ 5.0 & 0.53621 & 0.00307\end{array}$

Table ZG

Keffective Vs Uranium Concentration

55 Gallon Steel Drums filled with $\mathrm{UO}_{2} \mathrm{~F}_{2}$ - Water Mixture MG type concrete $10^{-4}$ (gm water/cc of air) mist density $200 \mathrm{~cm}$ Pitch 2\% Enrichment

$\begin{array}{ccc}\begin{array}{c}\text { Uranium Concentration } \\ \text { Grams U/cc Solution }\end{array} & \begin{array}{c}\text { Keffective } \\ \text { mean value }\end{array} & \begin{array}{c}1 \text { Sigma } \\ \text { value }\end{array} \\ 0.2 & 0.37518 & 0.00122 \\ 0.3 & 0.49451 & 0.00169 \\ 0.4 & 0.58558 & 0.00179 \\ 0.5 & 0.66506 & 0.00210 \\ 1.0 & 0.87197 & 0.00312 \\ 1.5 & 0.96089 & 0.00336 \\ 2.0 & 0.99265 & 0.00339 \\ 2.5 & 0.99476 & 0.00343 \\ 3.0 & 0.97677 & 0.00329 \\ 4.0 & 0.94062 & 0.00373 \\ 4.5 & 0.75801 & 0.00323 \\ 5.0 & 0.40291 & 0.00214\end{array}$


Table FH

K-effective Vs Uranium Concentration

55 Gallon Steel Drums filled with $\mathrm{UO}_{2} \mathrm{~F}_{2}$ - Water Mixture MG type concrete $\quad 10^{-4}$ ( $\mathrm{gm}$ water/cc of air) mist density $300 \mathrm{~cm}$ Pitch 5\% Enrichment

$\begin{array}{ccc}\begin{array}{c}\text { Uranium Concentration } \\ \text { Grams U/cc Solution }\end{array} & \begin{array}{c}\text { K-effective } \\ \text { mean value }\end{array} & \begin{array}{c}\text { Sigma } \\ \text { value }\end{array} \\ 0.2 & 0.69899 & 0.00223 \\ 0.3 & 0.84823 & 0.00262 \\ 0.4 & 0.94953 & 0.00308 \\ 0.5 & 1.01179 & 0.00333 \\ 1.0 & 1.17543 & 0.00378 \\ 1.5 & 1.20031 & 0.00440 \\ 2.0 & 1.20610 & 0.00419 \\ 2.5 & 1.16571 & 0.00435 \\ 3.0 & 1.12871 & 0.00456 \\ 4.0 & 1.06608 & 0.00411 \\ 4.5 & 0.87333 & 0.00375 \\ 5.0 & 0.47322 & 0.00270\end{array}$

Table ZH

K-effective Vs Uranium Concentration

55 Gallon Steel Drums filled with $\mathrm{UO}_{2} \mathrm{~F}_{2}$ - Water Mixture MG type concrete $\quad 10^{-4}$ (gm water/cc of air) mist density $300 \mathrm{~cm}$ Pitch 2\% Enrichment

$\begin{array}{ccc}\begin{array}{c}\text { Uranium Concentration } \\ \text { Grams U/cc Solution }\end{array} & \begin{array}{c}\text { Keffective } \\ \text { mean value }\end{array} & \begin{array}{c}\text { 1 Sigma } \\ \text { value }\end{array} \\ 0.2 & 0.37450 & 0.00134 \\ 0.3 & 0.49201 & 0.00162 \\ 0.4 & 0.58302 & 0.00186 \\ 0.5 & 0.65367 & 0.00235 \\ 1.0 & 0.87373 & 0.00308 \\ 1.5 & 0.95378 & 0.00337 \\ 2.0 & 0.98769 & 0.00343 \\ 2.5 & 0.97872 & 0.00379 \\ 3.0 & 0.96356 & 0.00373 \\ 4.0 & 0.93404 & 0.00405 \\ 4.5 & 0.73421 & 0.00417 \\ 5.0 & 0.34102 & 0.00209\end{array}$




\section{Table FI}

K-effective Vs Uranium Concentration

55 Gallon Steel Drums filled with $\mathrm{UO}_{2} \mathrm{~F}_{2}$ - Water Mixture MG type concrete $10^{-4}$ (gm water/cc of aiz) mist density $400 \mathrm{~cm}$ Pii $\cdot \mathrm{h} \quad$ 5\% Enricbsnent

$\begin{array}{ccc}\begin{array}{c}\text { Uranium Concentration } \\ \text { Grams U/cc Solution }\end{array} & \begin{array}{c}\text { Keffective } \\ \text { mean value }\end{array} & \begin{array}{c}1 \text { Sigma } \\ \text { value }\end{array} \\ 0.2 & 0.69540 & 0.00279 \\ 0.3 & 0.84335 & 0.00266 \\ 0.4 & 0.94684 & 0.00347 \\ 0.5 & 1.02255 & 0.00352 \\ 1.0 & 1.16745 & 0.00422 \\ 1.5 & 1.20475 & 0.00441 \\ 2.0 & 1.19027 & 0.00426 \\ 2.5 & 1.17173 & 0.00447 \\ 3.0 & 1.12013 & 0.00423 \\ 4.0 & 1.06839 & 0.00372 \\ 4.5 & 0.86641 & 0.00386 \\ 5.0 & 0.44977 & 0.00234\end{array}$

Table ZI

K-effective Vs Uranium Concentration

55 Gallon Steel Drums filled with $\mathrm{UO}_{2} \mathrm{~F}_{2}$ - Water Mixture MG type concrete $\quad 10^{-4}$ (gm water/cc of air) mist density $400 \mathrm{~cm}$ Pitch 2\% Enrichment

$\begin{array}{ccc}\begin{array}{c}\text { Uranium Concentration } \\ \text { Grams U/cc Solution }\end{array} & \begin{array}{c}\text { K-effective } \\ \text { mean value }\end{array} & \begin{array}{c}\text { 1 Sigma } \\ \text { value }\end{array} \\ 0.2 & 0.37050 & 0.00116 \\ 0.3 & 0.49030 & 0.00158 \\ 0.4 & 0.58104 & 0.00174 \\ 0.5 & 0.65542 & 0.00220 \\ 1.0 & 0.86352 & 0.00279 \\ 1.5 & 0.94630 & 0.00339 \\ 2.0 & 0.98311 & 0.00376 \\ 2.5 & 0.97765 & 0.00400 \\ 3.0 & 0.95974 & 0.00339 \\ 4.0 & 0.91251 & 0.00344 \\ 4.5 & 0.72300 & 0.00387 \\ 5.0 & 0.31738 & 0.00209\end{array}$


Table FJ

K-effective Vs Uranium Concentration

55 Gallon Steel Drums filled with $\mathrm{UO}_{2} \mathrm{~F}_{2}$ - Water Mixture MG type concrete $\quad 10^{-4}$ (gm water/cc of air) mist density $500 \mathrm{~cm}$ Pitch 5\% Enrichment

$\begin{array}{ccc}\begin{array}{c}\text { Uranium Concentration } \\ \text { Grams U/cc Solution }\end{array} & \begin{array}{c}\text { K-efective } \\ \text { mean value }\end{array} & \begin{array}{c}\text { Sigma } \\ \text { value }\end{array} \\ 0.2 & 0.69342 & 0.00239 \\ 0.3 & 0.84629 & 0.00292 \\ 0.4 & 0.94869 & 0.00359 \\ 0.5 & 1.01481 & 0.00362 \\ 1.0 & 1.16371 & 0.00434 \\ 1.5 & 1.19111 & 0.00391 \\ 2.0 & 1.19769 & 0.00433 \\ 2.5 & 1.16598 & 0.00372 \\ 3.0 & 1.10933 & 0.00446 \\ 4.0 & 1.05859 & 0.00418 \\ 4.5 & 0.85931 & 0.00402 \\ 5.0 & 0.43588 & 0.00232\end{array}$

Table ZJ

K-effective Vs Uranium Concentration

55 Gallon Steel Drums filled with $\mathrm{UO}_{2} \mathrm{~F}_{2}$ - Water Mixture MG type concrete $\quad 10^{-4}$ (gm water/cc of air) mist density $500 \mathrm{~cm}$ Pitch 2\% Enrichment

$\begin{array}{ccc}\begin{array}{c}\text { Uranium Concentration } \\ \text { Grams U/cc Solution }\end{array} & \begin{array}{c}\text { K-effective } \\ \text { mean value }\end{array} & \begin{array}{c}\text { 1 Sigma } \\ \text { value }\end{array} \\ 0.2 & 0.37037 & 0.00125 \\ 0.3 & 0.49257 & 0.00142 \\ 0.4 & 0.58492 & 0.00195 \\ 0.5 & 0.65527 & 0.00220 \\ 1.0 & 0.85878 & 0.00315 \\ 1.5 & 0.94735 & 0.00337 \\ 2.0 & 0.97193 & 0.00352 \\ 2.5 & 0.97530 & 0.00389 \\ 3.0 & 0.94602 & 0.00428 \\ 4.0 & 0.91181 & 0.00372 \\ 4.5 & 0.72003 & 0.00303 \\ 6.0 & 0.30717 & 0.00211\end{array}$



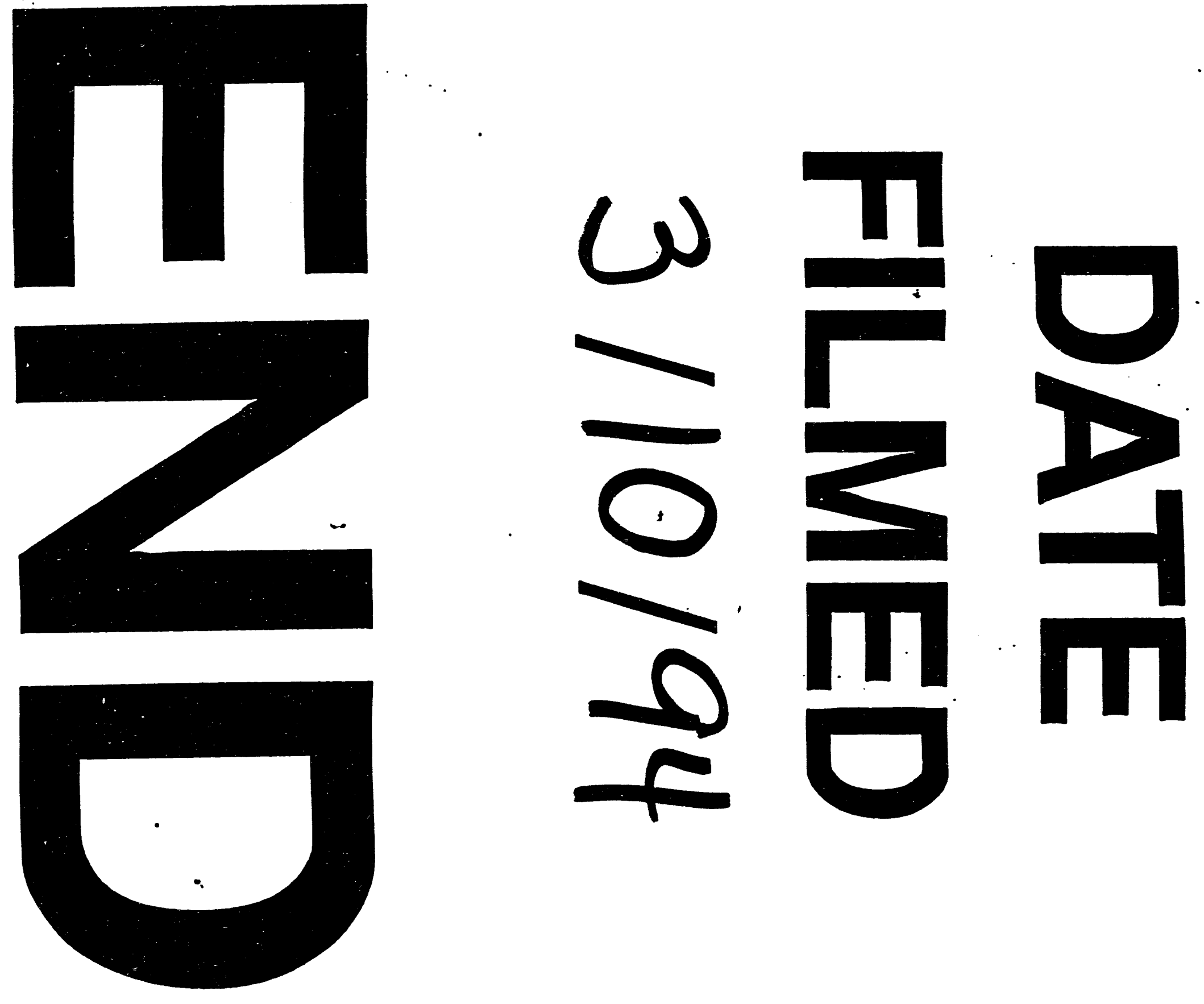
$$
\text { - }
$$$$
\text { (1) }
$$

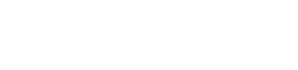

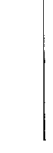

